Portland State University

PDXScholar

$11-29-1993$

\title{
Modeling and Simulation of Bipolar Transistor at Low Temperature
}

Swarupa Madhav Nerurkar

Portland State University

Follow this and additional works at: https://pdxscholar.library.pdx.edu/open_access_etds

Part of the Electrical and Electronics Commons

Let us know how access to this document benefits you.

\section{Recommended Citation}

Nerurkar, Swarupa Madhav, "Modeling and Simulation of Bipolar Transistor at Low Temperature" (1993). Dissertations and Theses. Paper 4614.

https://doi.org/10.15760/etd.6498

This Thesis is brought to you for free and open access. It has been accepted for inclusion in Dissertations and Theses by an authorized administrator of PDXScholar. Please contact us if we can make this document more accessible: pdxscholar@pdx.edu. 
THESIS APPROVAL

The abstract and thesis of _Swarupa Madhav Nerurkar for the M.S. degree in Electrical Fngineering

were presented November 29, 1993 and accepted by the thesis committee and the department.

COMMITTEE APPROVALS:
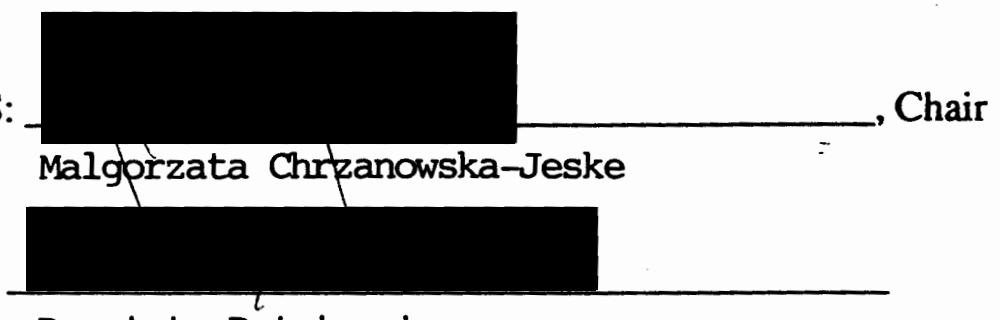

Branimir Pejcinovic

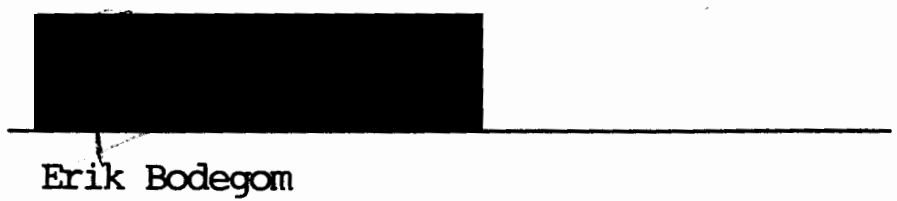

Representative of the Office of Graduate Studies

DEPARTMENT APPROVAL:

Rolf Schaumann, Chair

Department of Electrical Engineering

ACCEPTED FOR PORTLAND STATE UNIVERSITY BY THE LIBRARY

by

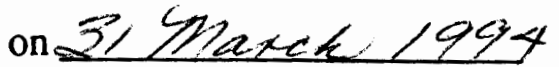




\begin{abstract}
An abstract of the thesis of Swarupa Madhav Nerurkar for the Masters of Science in Electrical Engineering presented November 29, 1993
\end{abstract}

Title: Modeling and Simulation of Bipolar Transistor at Low Temperature

The BICMOS technology which integrates the CMOS technology with bipolar technology has drawn considerable attention as an attractive VLSI technology because of the high speed performance and low power consumption of the BICMOS. However, continued down scaling of CMOS devices has caused increased concerns with problems such as latch up, hot carriers and short channel effect. Most of the above mentioned problems can be avoided by operating the CMOS at liquid-nitrogen temperature(LNT). At low-temperatures, the CMOS exhibits lower sub threshold leakage, higher carrier mobility (which yields improved speed performance), and a steeper logarithmic currentvoltage slope. On the other hand, the low-temperature operation of conventional silicon bipolar circuits has been generally dismissed as impractical because of the well known decrease in the current gain at low temperature. The present interest in integrated bipolarCMOS circuits, plus the prospect of increased reliability, lower wiring delay, and lower noise, has revised interest in low-temperature bipolar devices. In this context, it is therefore important to acquire accurate knowledge of the transistor properties at liquid nitrogen temperature. This can be done in two ways. One is through experimental lowtemperature measurements and the other by low-temperature device simulations. 
Existing room temperature numerical simulators are typically not useful for low temperature conditions. This is because the physical assumptions such as complete ionization, the parameter models and implementation methods for room temperature condition do not hold at low temperature. Therefore, we used BiLow - a steady state onedimensional Bipolar Low Temperature Simulator for the temperature range of 77K$300 \mathrm{~K}$. This simulator, originally written in FORTRAN, was converted to $C$ for the dual purpose of proper memory management and making further modifications easier. The focus of this research has been to model bandgap narrowing, incomplete ionization and Mott Transition at room and at low-temperature, evaluate the performance of the new BiLow and to derive conclusions on the BJT performance at LNT.

It was observed that the bandgap narrowing was independent of temperature for the entire range of majority carrier concentration. The effect of Mott transition on the abrupt decrease in the electron concentration in emitter has been taken care of by smoothing out the concentration profile in the emitter thereby providing a continuity in the region of Mott transition. Both the current gain $(\beta)$ and the frequency $\left(f_{\mathrm{T}}\right)$ values obtained from simulating the two new profiles were found to be smaller than those obtained using the original BiLow simulator, as the doping in the base is higher and the device sizes were smaller. Most of the degradation in $\beta$ and $\mathrm{f}_{\mathrm{T}}$ was found to occur below $150 \mathrm{~K}$. From the plots of the charge characteristics, we found that the total charge which is a strong function of temperature is more in the case of the profiles studied for this work than the total charge from the original BiLow simulator. 
MODELING AND SIMULATION OF BIPOLAR TRANSISTOR AT

LOW TEMPERATURE by
SWARUPA MADHAV NERURKAR

A thesis submitted in partial fulfillement of the requirements for the degree of

\section{MASTER OF SCIENCE \\ in}

ELECTRICAL ENGINEERING

Portland State University 1993 


\section{ACKNOWLEDGEMENT}

I wish to express my thanks and my gratitude to my advisor Dr. Malgorzata Chrzanowska-Jeske for her guidance and support for this thesis work as well as for her

encouragement throughout my master's program. Special thanks are due to Dr. Brano Pjenovic and Dr. Erik Bodegom for their interest and comments on this work. Not to forget the staff of the EE dept, who have been ever ready to lend a helping hand. Lastly a big thankyou to my husband, Madhav and my daughter Rahee for just being there. 


\section{TABLE OF CONTENTS}

PAGE

ACKNOWLEDGEMENT....................................................................... ii

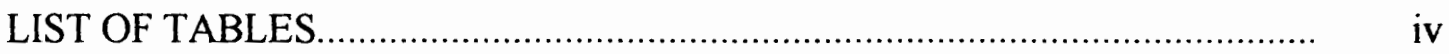

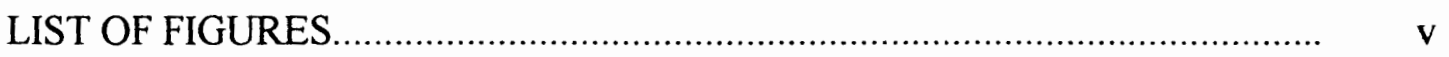

CHAPTER

I INTRODUCTION ...................................................................

II LOW TEMPERATURE SIMULATIONS........................................... 6

II.1 Basic Assumptions................................................................... 6

II.2 Physical Parameter Models ..................................................... 11

II.2.1 Poisson's Equation............................................. 11

II.2.2 Apparent Bandgap Narrowing Modeling .............. 12

II.2.3 Parameter Scaling ............................................ 14

II.2.4 Transport Equations........................................... 14

II.3 Enhancement To The Simulator …...................................... 15

III INCOMPLETE IONIZATION..................................................... 18

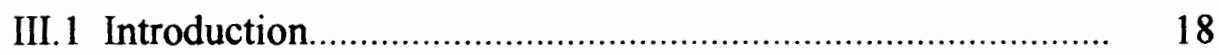

III.2 Theoretical Modeling.......................................................... 21

III.3 Results and Discussion......................................................... 27

IV BANDGAP NARROWING IN SILICON........................................ 31

IV.1 Background................................................................... 31

IV.2 Previous Work .................................................................... 32

IV.3 Method Used In This Study ................................................... 44

IV.3.1 Method I (Del Alamo et al BGN model [8]) .............. 45 
IV.3.2 Method II (Kuzmicz BGN model[27]) ........................ 46

IV.3.3 Method III (Original BGN model in BiLow [5]).......... 46

IV.4 Results ....................................................................... 48

$\mathrm{V}$ LOW TEMPERATURE BIPOLAR TRANSISTOR SIMULATIONS.... 53

V.1 Simulator Program Description................................................ 53

V.2 Simulator Performance........................................................... 54

V.3 Simulation Results............................................................... 55

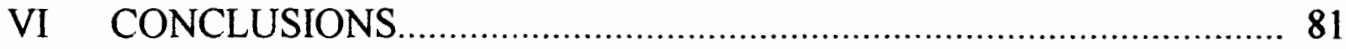

REFERENCES 


\section{LIST OF TABLES}

TABLE

PAGE

I Model parameters used in the interpretation of experimental data [23]

II Comparison of BGN model parameters …....................................... 36

III Convergence Statistics ……....................................................... 55 


\section{LIST OF FIGURES}

FIGURE

PAGE

1 One dimensional bipolar transistor device model $\quad 7$

2 Mesh definition 7

3 Flowchart for low temperature bipolar transistor simulations 13

4 Total ionized dopant distribution in the bipolar transistor at $\mathrm{T}=300 \mathrm{~K}$ $\begin{array}{ll}\text { and } \mathrm{T}=77[5] & 17\end{array}$

5 Ratio of ionized impurity as a function of donor concentration [17] 19

6 Incomplete ionization model flowchart 28

7 Ratio of the concentration of ionized impurity to the total doping concentration as a function of temperatureand doping 29

8 Total ionized dopant distribution in the bipolar transistor at $\mathrm{T}=300 \mathrm{~K}$ and $\mathrm{T}=77 \mathrm{~K}$ using the new BiLow.............................................................. 30

9 Summary of Bandgap narrowing from different measurements.

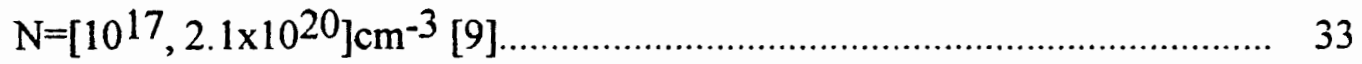

10 Bandgap narrowing as a function of impurity concentration [23]

$11 \Delta \mathrm{Eg}$ vs carrier concentration at $\mathrm{T}=20 \mathrm{~K}[23]$

$12 \Delta \mathrm{Eg}$ vs concentration at $\mathrm{T}=300 \mathrm{~K}[55]$. 38

13 BGN values in p-Si [20] 42

14 BGN values in $\mathrm{n}-\mathrm{Si}[20]$

15 Bandgap narrowing at $\mathrm{T}=300 \mathrm{~K}$ as a function of temperature 49

16 Bandgap narrowing as a function of ionized donor concentration and

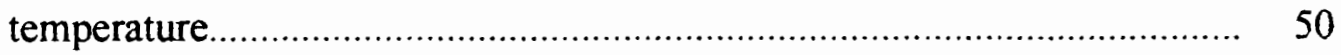


17 Bandgap narrowing as a function od donor concentration and temperture 51

18 Doping profile of an $n-p-n$ transistor $T 1$

19 (a) Charge characteristics at $\mathrm{T}=300 \mathrm{~K}$, $\mathrm{a}=$ total charge, $\mathrm{b}=$ charge of electron in the base, $c=$ charge of hole in emitter, $d=$ charge of donors trapped in base

(b) Charge characteristics at $T=150 \mathrm{~K}$, $a=$ total charge, $b=$ charge of electron in the base, $c=$ charge of hole in emitter, $d=$ charge of donors trapped in base

20 (a) Charge characteristics at $\mathrm{T}=122 \mathrm{~K}$, $\mathrm{a}=$ total charge, $\mathrm{b}=$ charge of electron in the base, $c=$ charge of hole in emitter, $d=$ charge of donors trapped in base

(b) Charge characteristics at $\mathrm{T}=100 \mathrm{~K}$, $a=$ total charge, $b=$ charge of electron in the base, $c=$ charge of hole in emitter, $d=$ charge of donors trapped in base

21 Minority carrier distribution in n-p-n transistor 59

22 Minority carrier distribution to explain the charge characteristics 60

23 n-p-n doping profile [6] 61

24 Charge characteristics at $\mathrm{T}=300 \mathrm{~K}$ for original BiLow [6] 61

25 Charge characteristics at $\mathrm{T}=122 \mathrm{~K}$ for original BiLow [6] 61

26 Charge characteristics at $\mathrm{T}=77 \mathrm{~K}$ for original BiLow [6] 61

27 Electron distribution at different temperatures for high-level condition $\mathrm{J}(300 \mathrm{~K})=480 \mathrm{~A} / \mathrm{cm}^{2}, \mathrm{~J}(122 \mathrm{~K})=800 \mathrm{~A} / \mathrm{cm}^{2}, \mathrm{~J}(100 \mathrm{~K})=65 \mathrm{~A} / \mathrm{cm}^{2}$

28 Electron distribution at different temperatures for high-level condition $\mathrm{J}(300 \mathrm{~K})=550 \mathrm{~A} / \mathrm{cm}^{2}, \mathrm{~J}(122 \mathrm{~K})=840 \mathrm{~A} / \mathrm{cm}^{2}, \mathrm{~J}(77 \mathrm{~K})=65 \mathrm{~A} / \mathrm{cm}^{2}[6] \ldots \ldots \ldots .64$

29 Temperature dependence of peak $f_{T}$

30 Simulated temperature dependence of peak $f_{T}$ for $n-p-n$ transistor [5] 66 
31 Unity gain frequency as a function of collector current density at different temperatures for $\mathrm{T} 1$..

32 Unity gain frequency as a function of temperature and collector current density from [5].

33 Temperature dependence of the ratio of the charge of electorns trapped on the donors in the base to the charge of free electrons in the base at peak $\mathrm{f}_{\mathrm{T}}$ for $\mathrm{T} 1$.

34 Current gain as a function of temperature and collector current density using the new BiLow for $\mathrm{T} 1$

35 Current gain as a function of temperature and collector current density from [5].

36 (a) Charge characteristics at $\mathrm{T}=300 \mathrm{~K}, \mathrm{a}=$ total charge, $\mathrm{b}=$ charge of electrons in base, $c=$ charge of holes in emittter, $d=$ cahrge of donors trapped in base using Method I.

(b) Charge characteristics at $\mathrm{T}=150 \mathrm{~K}, \mathrm{a}=$ total charge, $\mathrm{b}=$ charge of electrons in base, $c=$ charge of holes in emittter, $d=$ charge of donors trapped in base using Method I

37 (a) Charge characteristics at $\mathrm{T}=122 \mathrm{~K}, \mathrm{a}=$ total charge, $\mathrm{b}=$ charge of electrons in base, $c=$ charge of holes in emittter, $d=$ cahrge of donors trapped in base using Method I.

(b) Charge characteristics at $\mathrm{T}=100 \mathrm{~K} a=$ total charge, $\mathrm{b}=$ charge of electrons in base, $c=$ charge of holes in emittter, $d=$ cahrge of donors trapped in base using Method I.

38 Temperature dependence of current-gain for T1 using BGN Model I 72

39 Temperature dependence of $\mathrm{f}_{\mathrm{T}}$ for $\mathrm{T} 1 \mathrm{using}$ BGN Model I 73

40 (a) Charge characteristics at $T=300 \mathrm{~K}, \mathrm{a}=$ total charge, $\mathrm{b}=$ charge of electrons 
40 (a) Charge characteristics at $\mathrm{T}=300 \mathrm{~K}, \mathrm{a}=$ total charge, $\mathrm{b}=$ charge of electrons in base, $\mathrm{c}=$ charge of holes in emitter, $\mathrm{d}=$ charge of donor trapped in base using BGN Model III for T1

(b) Charge characteristics at $\mathrm{T}=150 \mathrm{~K}, \mathrm{a}=$ total charge, $\mathrm{b}=$ charge of electrons in base, $c=$ charge of holes in emitter, $d=$ charge of donor trapped in base using BGN Model III for T1.

41 (a) Charge characteristics at $\mathrm{T}=122 \mathrm{~K}$, $a=$ total charge, $b=$ charge of electrons in base, $c=$ charge of holes in emitter, $d=$ charge of donor trapped in base using BGN Model III for T1.

41 (b) Charge characteristics at $\mathrm{T}=100 \mathrm{~K}$, $\mathrm{a}=$ total charge, $\mathrm{b}=$ charge of electrons in base, $c=$ charge of holes in emitter, $d=$ charge of donor trapped in base using BGN Model III for T1.

42 Temperature dependence of current-gain for T1 using BGN Model III 76

43 Temperature dependence of $\mathrm{f}_{\mathrm{T}}$ for $\mathrm{T} 1$ using BGN Model III 77

44 Doping Profile of a Double Diffused n-p-n Transistor T2 78

45 Temperature dependence of $\mathrm{f}_{\mathrm{T}}$ for $\mathrm{T} 2 \quad 79$

46 (a) Charge characteristics at $\mathrm{T}=300 \mathrm{~K}$, $\mathrm{a}=$ total charge, $\mathrm{b}=$ charge of electrons in base, $c=$ charge of holes in emitter, $d=$ charge of donor trapped in base for $T 2$.

(b) Charge characteristics at $\mathrm{T}=77 \mathrm{~K}$, $a=$ total charge, $b=$ charge of electrons in base, $c=$ charge of holes in emitter, $d=$ charge of donor trapped in base for $\mathrm{T} 2$. 


\section{CHAPTER I}

\section{INTRODUCTION}

Novel fabrication methods have made a growing variety of electronic devices considerably more complex than earlier designs. Over the years, device size and critical dimensions have shrunk by orders of magnitude. Impurity doping densities have increased greatly and different spatial variation of impurity profile has become feasible. Yet, despite these advancements in semiconductor technology, physical understanding in the realm of small devices, highly doped devices, or devices with graded composition remains incomplete. The BICMOS technology which integrates the CMOS technology with bipolar technology has drawn considerable attention as an attractive VLSI technology because of the high speed performance and low power consumption [43] of the BICMOS. However, continued down scaling of CMOS devices has caused increased concerns with problems such as latch up, hot carriers and short channel effect. Most of the above mentioned problems can be avoided by operating the CMOS at liquid-nitrogen temperature(LNT). At low-temperatures, the CMOS exhibits lower sub threshold leakage, higher carrier mobility (which yields improved speed performance), and a steeper logarithmic current-voltage slope. On the other hand, the low-temperature operation of conventional silicon bipolar circuits has been generally dismissed as impractical because of the well known decrease in the current gain at low temperature [13]. The present interest in integrated bipolar-CMOS circuits, plus the prospect of increased reliability, lower wiring delay, and lower noise, has revised interest in lowtemperature bipolar devices. In this context, it is therefore important to acquire accurate 
knowledge of the transistor properties at liquid nitrogen temperature. This can be done in two ways. One is through experimental low-temperature measurements and the other by low-temperature device simulations. The accuracy of simulation largely depends on device modeling. Device modeling based on self-consistent solution of fundamental semiconductor equations dates back to the work of Gummel in 1964 [18]. However, the first application of this style of modeling for problems at low temperature was first carried out by Gaensslen et al in 1976 [17]. The main reason for this delay cannot only be seen in the lesser demands for low temperature simulation. The primary reason for the fairly poor status in fully numerical low-temperature device simulation stems from considerably increased difficulties regarding physical assumptions and implementation of the numerical solution [45].

Existing room temperature numerical simulators are typically not useful for low temperature conditions. This is because the physical assumptions such as complete ionization, the parameter models and implementation methods for room temperature condition do not hold at low temperature. Therefore, we used BiLow [5]- a steady state one-dimensional Bipolar Low Temperature Simulator for the temperature range of $77 \mathrm{~K}$ 300K. This simulator, originally written in FORTRAN, was converted to $C$ for the dual purpose of proper memory management and making further modifications easier. In the process, iterative loops were simplified to make experimenting with different models simpler. The availability of dynamic memory allocation facility of the C-language can be used to easily extend it so that large problems can be accommodated without the smaller problems having to pay the penalty of huge unused memory. Widespread availability of C on different platforms (including PCs) and extensive C literacy of EE students should make continued refinement of this program possible. Besides, the need to evaluate and update the models used in the simulator is always necessary. 
Transistors have changed a great deal in the last ten years. The work of Schlig [44] and the interpretation by Dumke [13] were based on diffused transistors, whereas the self-aligned transistors still predominate [38]. The advent of self-aligned process, and the incorporation of the polysilicon emitter contact, results in transistors with higher current gain [38]. This makes scaling of the vertical dimensions possible, leading to smaller and faster transistors. This in turn demands greater base doping. At low-temperature, these changes affect among other things, two of the most important figures of merit predicting circuit performance, namely current gain $(\beta)$ and cutoff frequency $\left(f_{T}\right)$. The difference in the apparent bandgap narrowing (BGN) between the base and the emitter of a BJT is a very important factor in $\beta$ degradation at low temperature. Most of the discrepancies in the BGN values arise from the differences in the assumptions made about the minorityand majority-carrier mobility's and in some cases, from the lifetime values used in the interpretation of the experimental data. Because of the significance in a wide variety of application, considerable effort has been expended to obtain empirical information on the magnitude of the bandgap narrowing effect. A comparison between theoretical [23] and experimental [9]-[10], [26] BGN data shows a difference of upto $60 \%$ between them.

The lack of comparison of simulated to experimental data of some device parameter models necessitates the evaluation of the existing models and their relative impact on device simulations over temperatures. In this work, we have tried to compare the available literature data on bandgap narrowing so as to come up with a model of BGN for a wide range of doping concentrations. We have presented the literature survey in two groups. Group 1 talks about the optical and experimental work on BGN and Group 2 deals with the theoretical works of various authors. After evaluating a lot of models we have chosen two BGN models, one as described by del Alamo [58] and the other described by Kuzmicz [27]. Both the models are described by an analytical expression which can be 
easily implemented in the simulator. Although, Fermi-Dirac statistic has been used in the original version of Bilow, the bandgap narrowing parameters were evaluated using Boltzman statistics. Therefore, we have modified the BGN model used in the original version by adding a correction to account for Fermi-Dirac statistics. In the BGN models used, we have assumed that the majority carrier concentration is equal to the ionized impurity concentration. Therefore, it is important to accurately model the ionized impurities as the BGN model is influenced by them.

Another important phenomena that needs careful attention during low-temperature simulations is that of incomplete ionization. For most room temperature applications complete ionization of impurities is assumed. However, at low-temperatures only a small fraction of the impurity atoms are ionized. In addition, at high doping concentration all impurity atoms get ionized independently of temperature and the semiconductor starts acting like a metal. This phenomenon is known as Mott transition. This necessitates the proper modeling of these two phenomena. In order to incorporate the incomplete ionization and Mott transition into the realm of device simulations, we have studied the incomplete ionization model proposed by Wieslaw Kuzmicz [25]. We evaluated this model at room and at low-temperature and then included it into the simulator. It is also very important for the bandgap narrowing calculations to include the concentration of ionized impurities, as the BGN models assumed that the majority carrier concentration is equal to the ionized impurity concentration. We have used the incomplete ionization and Mott transition data to extract the $\mathrm{BGN}$ values as the bandgap narrowing models use majority-carrier concentration in the calculation of the $B G N$ parameters.

The focus of this research has been to model bandgap narrowing, incomplete ionization and Mott Transition at room and at low-temperature, evaluate the performance of the new BiLow and to derive conclusions on the BJT performance at LNT. Chapter II talks about the existing models, their drawbacks and the basic assumptions used in the 
simulator BiLow. The incomplete ionization model including the Mott transition model has been described and the results presented in Chapter III. The performance of two models for bandgap narrowing is compared along with the existing model in the simulator in Chapter IV. The results from the new simulator are presented in the final Chapter V. 


\section{CHAPTER II}

\section{LOW TEMPERATURE SIMULATION}

\subsection{Basic Assumptions}

The performance of the bipolar transistor at low temperature depends on the modeling of the physical parameters of Poisson's equation and the electron and hole transport equations. In this section we give a brief description of the basic assumptions made, and the parameter models used by the original BiLow [5]. A one-dimensional simulator, was chosen since it can successfully simulate much of the overall bipolar transistor behavior.

In order to describe the performance of semiconductor devices at low temperature, the electron and hole concentrations, and the electrostatic potential, must be calculated using these semiconductor equations of state [45]:

$$
\begin{aligned}
& \frac{d^{2} \psi}{d x^{2}}-\frac{q}{E\left(n-p+N_{A}^{-}-N_{D}^{+}\right)}=0 \\
& \frac{1}{q} \frac{d J_{n}}{d x}-R_{\text {net }}=0 \\
& \frac{1}{q} \frac{d J_{p}}{d x}-R_{\text {net }}=0 \\
& J_{n}=-q D_{n} \frac{d n}{d x}-q \mu_{n} n E \\
& J_{p}=q D_{p} \frac{d p}{d x}+q \mu_{p} p E
\end{aligned}
$$


Where eqn.(1) is Poisson's equation, and eqn.(2) and (3) are the continuity equations and (4) and (5) are the current densities for electrons and holes respectively. In the above equations, $\mathrm{n}$ and $\mathrm{p}$ are the electron and hole carrier concentrations, $\psi$ is the electrostatic voltage potential, $\mathrm{ND}^{+}$and $\mathrm{N}_{\mathrm{A}}^{-}$are the ionized donor and acceptor impurity dopant concentrations, $J_{n}$ and $J_{p}$ are the electron and hole current densities, $R_{n e t}$ represents the net recombination rate, $\mathrm{x}$ is the position coordinate $\mu$ is the mobility, $\mathrm{E}$ is the electric field and $D_{n}$ and $D_{p}$ represent the diffusion length of electrons and holes respectively. For numerical implementation, eqns. (1) - (3) are normalized and discretized on a onedimensional non-uniform mesh. Due to normalization, eqns. (1) - (3) not only result in simpler, unitless equations, but it also decreases the numerical error that might otherwise occur. The equations are discretized using finite difference technique.

The one dimensional structure used by BiLow [5] is shown in Fig 1.

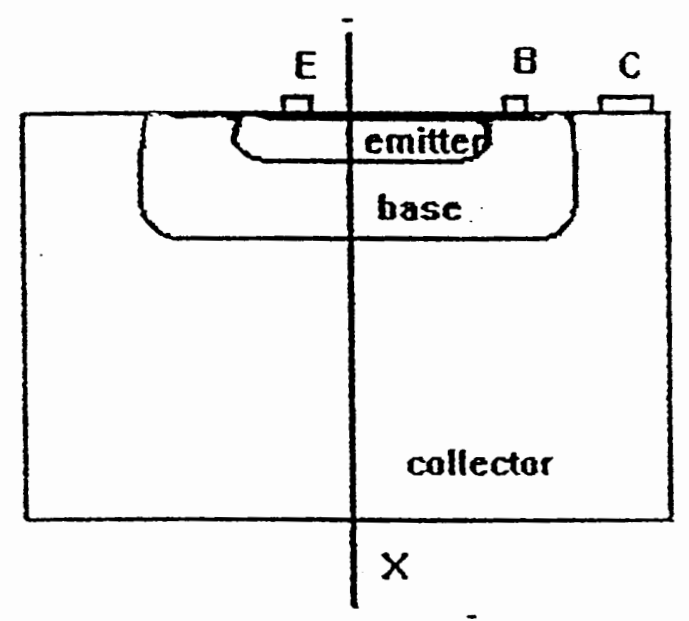

Figure 1. One Dimensional Bipolar Transistor Device Model 
The definition of the mesh used in the original BiLow is illustrated in Fig 2. The mesh points defined as auxillary midpoints such as point $\mathrm{K}$ are located midway between nodes $\mathrm{N}$ and $\mathrm{N}+1$. Because of the nature of the problem, the nodes are spaced non uniformly, based on the knowledge of the dependent variable distribution along the structure, which must be provided by the user.

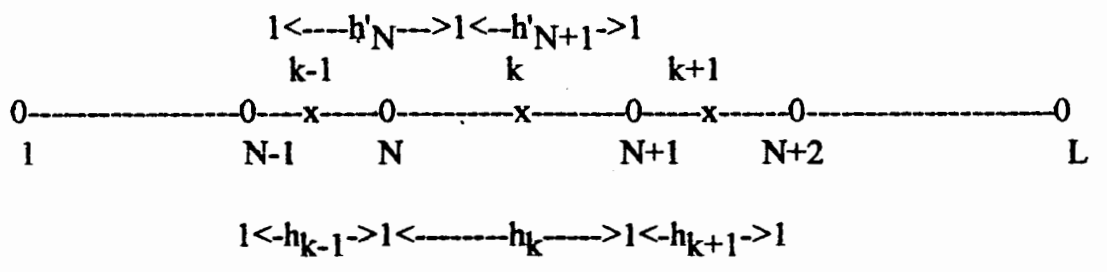

Figure 2. Mesh Definition

After normalization and discretization, eqns.(1), (2) and (3) are transformed into the following set of difference equations [45] :

$$
\begin{aligned}
& \frac{h_{k-1} \psi_{N+1}-2 h_{N}^{\prime} \psi_{N}+h_{k} \psi_{N-1}}{h_{k} h_{k-1} h_{N}^{\prime}}=n_{N}-p_{N}+N_{A N^{-}}-N_{D N^{+}} \\
& \frac{J_{n k}-J_{n k-1}}{h_{N}^{\prime}}=+R_{n e t} N \\
& \frac{J_{p k}-J_{p k-1}}{h_{N}^{\prime}}=-R_{n e t} N
\end{aligned}
$$

where the subscripts indicate at which nodes or auxillary midpoints the quantities in the equations are evaluated. For example, $\mathrm{n}_{\mathrm{N}}$ represents the normalized electron concentration at node $\mathrm{N}$. 
Following the work of Gummel [3], the electron and hole current density expressions were represented in an integral form to increase stability. This yields the following pair of discretized equations:

$$
\begin{aligned}
& J_{n k}=\frac{q}{h_{k}} D_{n k}\left[B\left(V_{n k}\right) n_{N}-B\left(-V_{n k}\right) n_{N+1}\right] \\
& J_{p k}=\frac{q}{h_{k}} D_{p k}\left[B\left(-V_{p k}\right) p_{N}-B\left(V_{p k}\right) p_{N+1}\right]
\end{aligned}
$$

where

$$
V_{n k}=V_{p k}=\frac{q}{k T}\left\{\psi(N) \psi(N+1)^{\frac{1}{2}}[T(N)-T(N+1)]\right\}
$$

and $\quad B(Y)=\frac{Y}{e^{Y}-1}$

is called the Bernulli function. The numerical implementation of the Bernulli function is taken from Selberherr [45]. In order to obtain eqns. (9) and (10), the electric field, current density and mobility were assumed to be constant in the range of $x$ between mesh points $\mathrm{N}$ and $\mathrm{N}+1$.

After discretization on a one-dimensional non uniform mesh, the system of equations can be represented as:

$$
\begin{aligned}
& F_{\psi}(\psi, n, p)=0 \\
& F_{n}(\psi, n, p)=0 \\
& F_{p}(\psi, n, p)=0
\end{aligned}
$$

Each of the differential equations are treated separately by decoupling the system and solving eqns. (13)-(15) successfully. The Poisson eqn. (13) is solved assuming known electron and hole concentration, $\mathrm{n}$ and $\mathrm{p}$. Then comes the solution for each of the continuity equations with $\psi$ given from the first step. To solve each of the non-linear equations from eqns.(13)-(15), an iterative method is chosen due to its quadratic 
convergence. A substantial reduction in CPU time can be achieved by this method. Applying this method to Poisson's equation, the system (13) is linearized and the iteration process is defined as :

$$
\frac{d F_{\psi}}{d \psi} d \psi_{k+1}=-F_{\psi}\left(x_{k}\right) \quad \psi_{\mathbf{k}+1}=\psi_{\mathbf{k}}+\mathrm{d} \psi_{\mathrm{k}+1} ; \quad \mathrm{k}=0,1,2 \ldots
$$

$\frac{d F_{\psi}}{d \psi}$ is the Jacobian matrix evaluated at $\psi_{\mathbf{k}}$ and $d \psi_{\mathbf{k}+1}$ is the correction vector of iteration step $k+1$. This simple implementation of the Newton's method results in slow convergence if eqns. (13)-(15) are strongly coupled. The coupled and decoupled methods can be combined into a hybrid solution method [45], which starts with the decoupled method and switches to the coupled one if the solution is not reached after a certain number of iterations. The hybrid approach has been succesfully used in BiLow, and the flowchart for the operation of the simulator is given in Fig 3. The carrier and potential distribution for one bias point is used as initial condition for a new bias point for the coupled solver. If these values are not available, the simulator starts using the decoupled solver with zero bias condition and then increments the bias voltage in small steps using the coupled solver until the desired bias conditions are achieved. The relative errors $\frac{d p}{p}$, $\frac{d n}{n}$, and $\frac{d \psi}{\psi}$ are used as basic convergence criteria. The relative errors between two successive Newton iterations has to be less than $10^{-4}$. The accuracy of the simulation is checked by calculating the total current density, which should be constant through the device with relative error less than $10^{-4}$. For the one-dimensional simulator, the total current density is discontinuous at the base contact which is taken to be at the midpoint of the base. For the emitter side, the current density is equal to the emitter current density and for the collector side it is assumed to be equal to the collector current density. 


\section{II.2 Physical Paramater models for Low Temperture Simulation}

In the following section, we present the space charge model necessary to fully describe Poisson equation. A short description of the bandgap modeling parameters followed by the parameters used for scaling [5] are presented below.

\section{II.2.1 Poisson's Equation}

Poisson's equation which relates the electrostatic potential to the space charge can be represented as the sum of all the charges existing in the semiconductor [45]. This is given below as

$$
\rho=q\left(p-n+N_{D^{+}}-N_{A^{-}}\right)
$$

where $\mathrm{n}$ and $\mathrm{p}$ represent the electron and hole concentrations, $\rho$ is the potential. $\mathrm{ND}^{+}$and $\mathrm{N}_{\mathrm{A}}{ }^{-}$represent the ionized donor and acceptor concentrations respectively and are modeled by eqns. (31) and (32). For heavy doping concentrations the effective donor and acceptor ionization energies decrease as given in eqns. (33) and (34) for phosphorus and boron respectively [47]. An iterative solution techniques must be used to find $\mathrm{ND}^{+}$and $N_{A}-$ that satisfy eqns. (1) $-(6) . E_{F n}$ and $E_{F p}$ are the Quasi -Fermi levels and they are related to the electron and hole concentration through Fermi statistics. An iterative solution techniques must be used to find $\mathrm{ND}^{+}$and $\mathrm{N}_{A}^{-}$that satisfy eqns. (1) - (6). $\mathrm{E}_{\mathrm{Fn}}$ and $E_{F p}$ are the Quasi -Fermi levels and they are related to the electron and hole concentration through Fermi statistics.

$$
n=\frac{2 N_{c}}{\Delta F_{\frac{1}{2}}\left[\frac{\left(E_{F_{n}}-E_{c}\right)}{k T}\right]}
$$




$$
p=\frac{2 N_{v}}{\Delta F_{\frac{1}{2}}\left[\frac{\left(E_{V}-E_{F p}\right)}{k T}\right]}
$$

$\mathrm{N}_{\mathrm{C}}$ and $\mathrm{N}_{\mathrm{V}}$ are the density of states in the conduction and valence band and are given by eqns. (20) and (21).

$$
\begin{aligned}
& N_{c}=2\left(\frac{2 \pi k T m_{c}^{*}}{h^{2}}\right)^{\frac{3}{2}} \\
& N_{V}=2\left(\frac{2 \pi k T m_{V}^{*}}{h^{2}}\right)^{\frac{3}{2}}
\end{aligned}
$$

The effective masses for the electron $m_{c}$ and hole $m_{v}$ are modeled after the experimental work of Barber [15] and are valid over the temperature range of $50-300 \mathrm{~K}$.

$$
\begin{aligned}
& m_{c}=1.045+4.500 \times 10^{-4} \mathrm{~T} \\
& m_{V}=0.523+1.400 \times 10^{-3} \mathrm{~T}-1.480 \times 10^{-6} \mathrm{~T}^{2}
\end{aligned}
$$

\section{II.2.2 Apparant Bandgap Narrowing Modeling}

The product of the equilibrium hole and electron concentrations ( $p_{0}$ and $n_{0}$ respectively) in low doped semiconductors is a constant that depends only on temperature. That constant, $\mathrm{n}_{\mathrm{io}}$ is the intrinsic carrier concentration. The intrinsic concentration can be represented as a function of the density of states and the band width.

$$
\begin{aligned}
& \mathrm{p}_{\mathrm{o}} \mathrm{n}_{\mathrm{o}}=\mathrm{n}_{\mathrm{io}}{ }^{2}(\mathrm{~T}) \\
& n_{i o}=N_{c} N_{V}\left(\frac{-E_{\mathrm{g}}}{2 k T}\right)
\end{aligned}
$$

The numerical model of $n_{i}$ given by (25) is taken from Barber [15]

$$
n_{i o}=2.5 \times 10^{19}\left(m_{c} m_{V}\right)^{\frac{3}{4}}\left(\frac{T}{300}\right)^{1.5} \exp \left(\frac{-E_{g}}{2 k T}\right)
$$


The temperature dependence for the bandgap $\mathrm{Eg}=\mathrm{Ec}-\mathrm{Ev}$ is taken from Bladau et al [3].

For $\mathrm{T}<170 \mathrm{k}, \quad \mathrm{E}_{\mathrm{g}}=1.170+1.059 \times 10^{-5} \mathrm{~T}-6.050 \times 10^{-7} \mathrm{~T}^{2}$

For $\mathrm{T}>170 \mathrm{k} \quad \mathrm{E}_{\mathrm{g}}=1.179-9.025 \times 10^{-5} \mathrm{~T}-3.050 \times 10^{-7} \mathrm{~T}^{2}$

In heavily doped material, there is an increase in the $p_{o} n_{0}$ product due to substantial changes in the band structure. One way to treat the problem is to assume that a rigid narrowing of the bandgap is solely responsible for the increase of the minority-carrier concentration. The majority-carrier concentration is assumed to be equal to the ionized dopant concentration. In n-type silicon we can write,

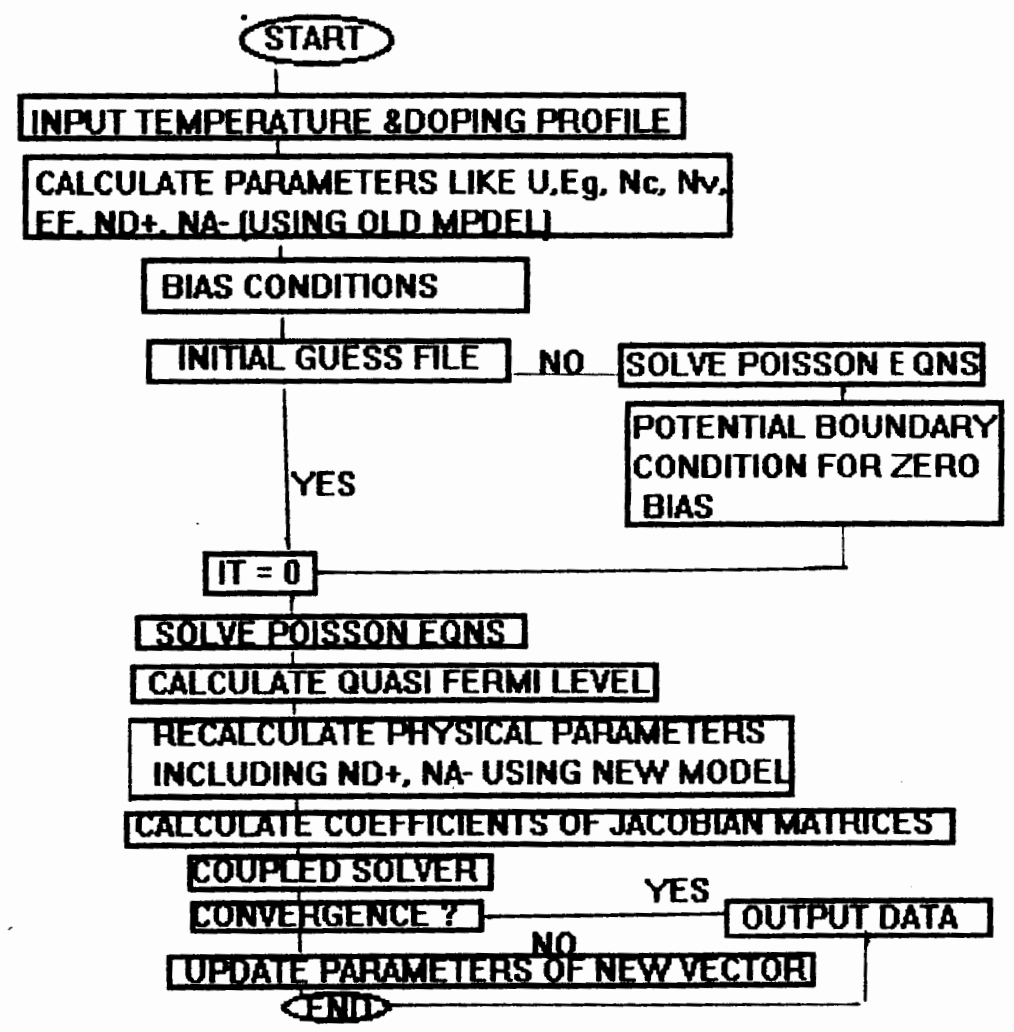

Figure 3. Flowchart for low temperature bipolar transistor simulations 


$$
p_{0} N_{D}^{+}=n_{i e}^{2}\left(N_{D}, T\right)=n_{i o}^{2} \exp \left[\frac{\Delta E_{g}^{a p p}\left(N_{D}, T\right)}{k T}\right]
$$

where $\Delta \mathrm{Eg}$ app represents the bandgap shrinkage that would account for the increase of $\mathrm{p}_{0}$ if no other heavy doping effect occured. The model used in the original version of BiLow [5] is presented in section II.3.

\section{II.2.3 Parameter Scaling}

Since the dependent variables (U,n,p) in the basic semiconductor eqns (1)-(5) are of greatly different orders of magnitude and show a strongly different behaviour in regions with small and large space charge, the first step towards a structural analysis of these equations is appropriate scaling. Scaling, which was introduced by DeMari [12], transformed the basic semiconductor equations into a dimensionless form, which was convenient for computer simulation. The scaling factor proposed by Selberherr [45], for low temperature modeling, has been been used in the original BiLow [5] and in the new version.

$$
\text { ninor }=4.8 \times 10^{22} \mathrm{n}_{\mathrm{io}}
$$

the constant $4.8 \times 10^{22} \mathrm{~cm}^{-3}$ is the number of silicon atoms per cubic centimeter and represents an absolute upper limit for the carrier concentration of any type. At $300 \mathrm{~K}$, ninor $=2.58 \times 10^{16} \mathrm{~cm}^{-3}$ and at $77 \mathrm{~K}$, ninor $=3.51 \times 10^{1} \mathrm{~cm}^{-3}$.

\section{II.2.4 Transport Equations.}

Another set of physical parameters that have to be carefully modeled for low temperature simulation consists of the transport and continuity equation parameters for both electrons and holes. The recombinetion rate $\mathrm{R}$ and the mobilities $\mu_{\mathrm{n}}$ and $\mu_{\mathrm{p}}$ are dependent on the semiconductor material, local values of carrier densities, electric field, and temperature. For the full set of equations, a relation between carrier density, the 
corresponding quasi-Fermi potential, and electrostatic potential is needed. The models for recombination and mobility are given in details by Selberherr [45] and are used in the original BiLow [5].

\section{II.3 Enhancement To The Simulator}

In the original version of BiLow [5] low-temperature parameter models for the effects such as bandgap narrowing, impurity freeze-out, temperature and doping dependent mobility were developed and are described in [6]. In line with the progress in lowtemperature simulation area [23], two main enhancements were made to the simulator program. In BiLow [5], the temperature dependence of the ionized impurity atoms $\mathrm{N}_{\mathrm{A}^{-}}$ and $\mathrm{ND}^{+}$was modeled by the classical formula using Fermi-Dirac statistic with appropriate degeneracy factors for electrons and holes in the conduction and valence band respectively.

$$
N_{D}^{+}=\frac{N_{D}}{1+2 \exp \left(\frac{E_{F n}-E_{D}}{k T}\right)}
$$

and $\quad N_{A}^{-}=\frac{N_{A}}{1+4 \exp \left(\frac{E_{A}-E_{F p}}{k T}\right)}$

Here the donor and the acceptor energies are given by

$$
\begin{aligned}
& E_{D}=0.045-3.6 \times 10^{-8}\left(N_{\text {Tion }}\right)^{1 / 3} \\
& E_{A}=0.0438-4.08 \times 10^{-8}\left(N_{\text {Tion }}\right)^{1 / 3}
\end{aligned}
$$

where $\mathrm{N}_{\text {Tion }}=\mathrm{N}_{\mathrm{D}}{ }^{+}+\mathrm{N}_{A^{-}}$

Equations (31) and (32) fit measured ionized impurity concentration reasonably well for donor and acceptor impurity concentration less than or equal to $10^{17} \mathrm{~cm}^{-3}$. However, at doping concentration greater than $10^{17} \mathrm{~cm}^{-3}$, neighbouring impurities in the 
semiconductor are now close enough that the electron wave functions at the impurity states overlap, resulting in their initial discrete energy levels changing into energy bands. Moreover, as a result of random distribution of impurities, fluctuations occur in the electrostatic potential throughout the crystal. Consequently there is some spatial fluctuation in the value of the conduction, valence, and impurity bands, thereby giving rise to bandtails. In addition, interaction between impurities and carriers and between ionized impurities and carriers results in the shift of the conduction, valence and impurity bands. From the simulation results using the original BiLow [5], one can see in Fig 4 the abrupt decrease in the electron concentration in the emitter, which becomes more visible at low-temperature. This is due to the improper modeling of Mott transition. Therefore, it has been our goal to study the ionization of impurity atoms and to come up with a continuous model to account for Mott tansition. An improved ionization model based on Wieslaw Kuzmicz model [25] which accounts for incomplete ionization and Mott transition was verified at low-temperature and then added to the simulator. The Kuzmicz model along with the results are disscused in Chapter III.

Lot of discrepancies have been reported between the experimental [8]-[10], [26], [58] and theoretical [2], [20], [31], [32] and [46] data on bandgap narrowing(BGN). Bandgap narrowing is a very important factor in the current-gain $(\beta)$ degradation and is responsible for the sharp decrease in $\beta$ at low-temperature. Therefore, a better understanding and modeling of the bandgap narrowing parameter has to be developed. We have added two separate models for BGN in the temperature range of $300-77 \mathrm{~K}$. The expression for BGN used in the original simulator [5] is as follows

$$
\Delta E_{g}=A \ln \left(\frac{N_{T}}{N_{\text {ref }}}\right)
$$

where $N_{\text {ref }}=N_{\text {ref }}(300 K) \frac{T}{300}\left\{1+2 \exp \left(\frac{E_{F_{n}}-E_{D}}{k T}\right)\right\} \quad$ for n-type 


$$
N_{r e f}=N_{r e f}(300 K) \frac{T}{300}\left\{1+4 \exp \left(\frac{E_{A}-E_{F p}}{k T}\right)\right\} \quad \text { for p-type }
$$

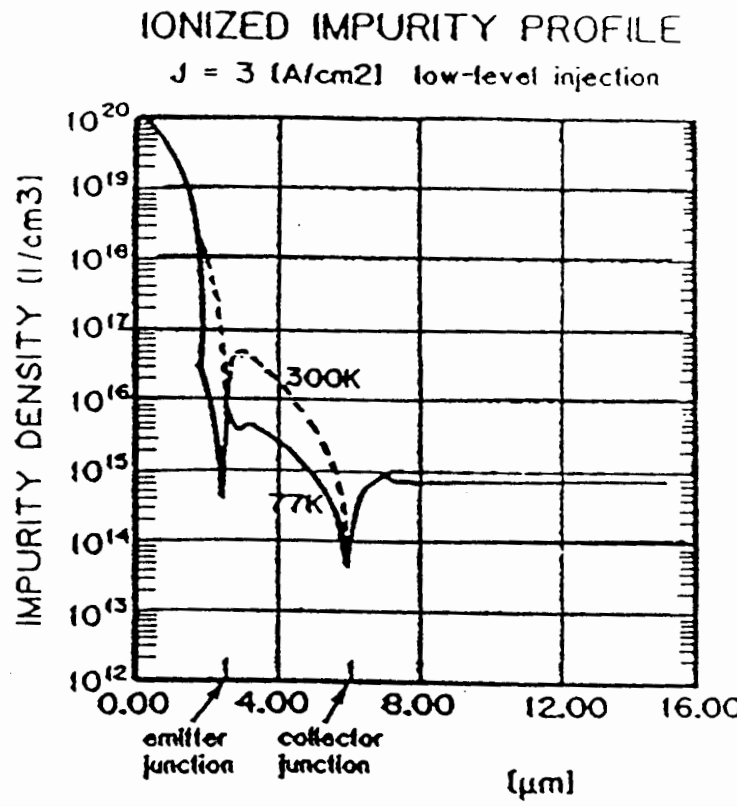

Figure 4. Total ionized dopant distribution in the transistor at $\mathrm{T}=300 \mathrm{~K}$ and at $\mathrm{T}=77 \mathrm{~K}[5]$

A Fermi-Dirac correction was added to the above expression of BGN. The BGN models are presented in Chapter IV. 
Chapter III

\section{INCOMPLETE IONIZATION}

\section{III.1 Introduction}

The incomplete ionization of a dopant impurity in a semiconductor is well understood for the case where the impurity concentration is low [23]. This is because the energy levels are discrete, and the band stucture is unaltered from the intrinsic case [23]. It becomes fairly simple, then, to count the total number of available energy levels [23]. However at concentrations above $10^{18} \mathrm{~cm}^{-3}$, several effects come into play making the situation more complicated. The initial discrete energy levels now change into energy bands because the electron wave functions at the impurity states overlap as the neighboring impurities get closer. As the impurity concentration increases, the impurity band enters the majority carrier band and the concept of separate band loses meaning. It then becomes critical to model this phenomenon properly. Theoretical calculations and measurements on silicon [23], indicate that in silicon in the doping range from about $10^{17} \mathrm{~cm}^{-3}$ to about $10^{19} \mathrm{~cm}^{-3}$, at room temperature and below, the dopant atoms are not completely ionized and the majority carrier concentration is significantly less than the dopant concentration. It is usually assumed that all dopants at room temperature are ionized. At low temperatures this assumption may lead to significant inaccuracies in the prediction of the device parameters. Therefore, it becomes necessary to develop a relationship between the concentration of ionized dopant atoms and the total doping concentration. Klassen and De Graff [23] have calculated the percentage of ionized 
dopant atoms in boron-doped Si and phosphorus-doped Si for the doping concentration upto $3 \times 10^{18} \mathrm{~cm}^{-3}$ and in the temperature range of $300 \mathrm{~K}-50 \mathrm{~K}$.

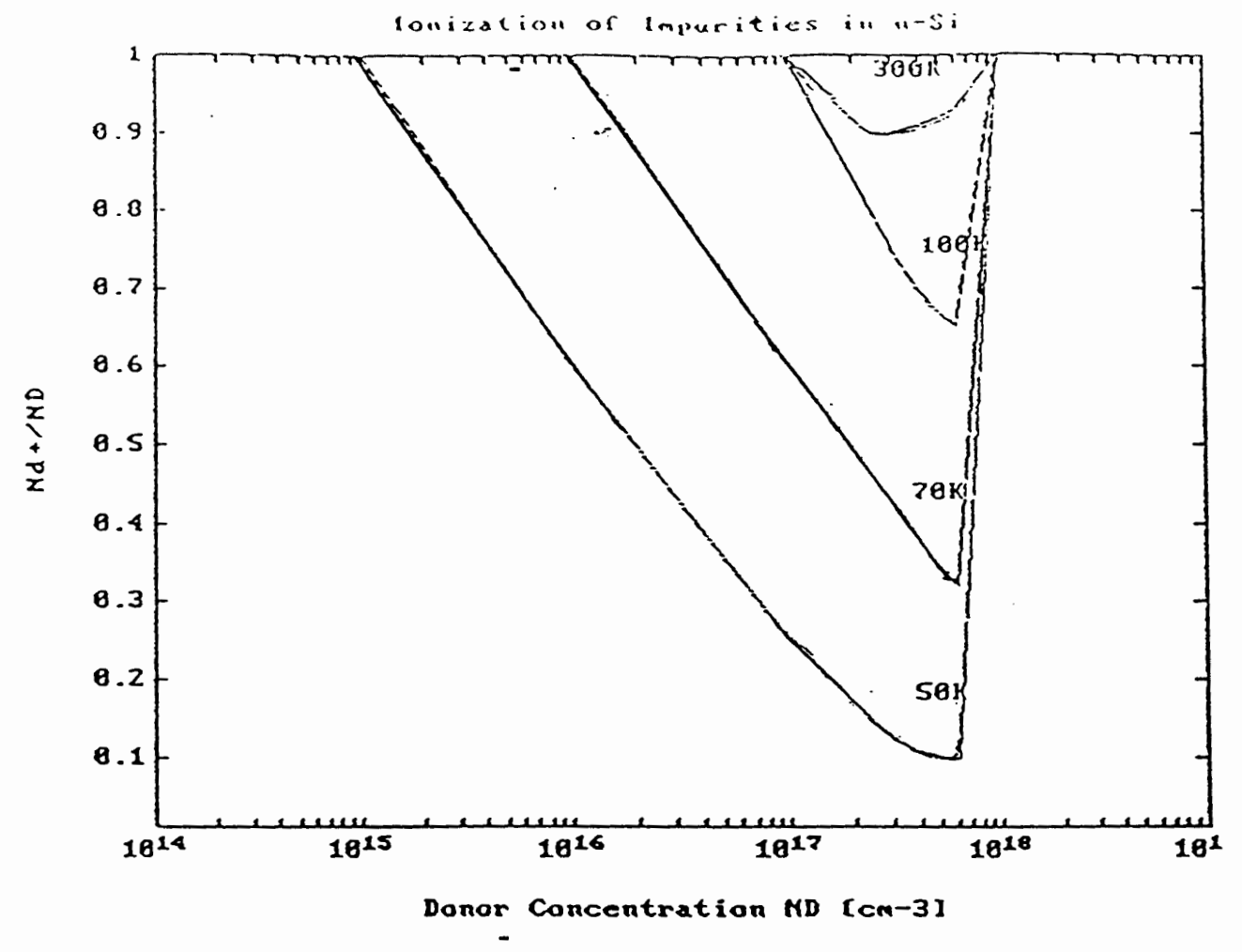

Figure 5. Ionized impurity ratio versus donor concentration [17]

The Klassen et al model [23] is based on a simple single-impurity-level theory, with the assumption that the activation energy of the donor or the acceptor level decreases with doping and eventually vanishes as the doping concentration approaches $3 \times 10^{18} \mathrm{~cm}^{-3}$. The model [23] uses eqns (31)-(35) to calculate the ionized donor and acceptor concentrations. The results from the Klassen et al [23] model are presented in Fig 5 which plots the ratio of ionized impurity to the donor concentration as a function of donor concentation for different temperature. As the doping concentration $\mathrm{N}_{\mathrm{d}}$ increases, the ionization energy of the impurity level decreases [23] and complete ionization is achieved at donor concentration of $10^{18} \mathrm{~cm}^{-3}$. Moreover, at high values of $\mathrm{N}_{\mathrm{d}}$ there is the 
broadening of the donor level into the impurity band, that will overlap with the conduction band. This effect enhances the ionization in such a way that that for large doping concentration of $10^{18} \mathrm{~cm}^{-3}, \Delta E d$ in eqn (34) goes to zero and $\mathrm{N}_{\mathrm{d}}{ }^{+}=\mathrm{N}_{\mathrm{d}}$. In Fig 5 this can be clearly seen as complete ionization is achieved at $10^{18} \mathrm{~cm}^{-3}$. The phenomenon of un-ionized impurity dopant is the strongest for doping distribution between $10^{17}$ and $3 \times 10^{18} \mathrm{~cm}^{-3}$. However, in this range of concentration the ionization is still incomplete. At the doping concentration that corresponds to zero activation energy, the concentration of ionized dopant atoms is neither equal to the total doping concentration nor temperature independent. Therefore, the model of Klassen and De Graff may be used at doping concentration well below $10^{18} \mathrm{~cm}^{-3}$ only.

To model incomplete ionization in the new version of BiLow we have selected the model proposed by Kuzmicz [25]. He has presented an analytical approximation for the ionized fraction as a function of temperature. This expression is shown [25] to be very useful in device modeling, especially for estimation of the temperature dependencies of device parameters. His results are based on a more sophisticated method and show complete ionzation at high concentrations and can be used over a wide range of concentration. The model includes the change of activation energy with the doping as well as the broadening of the impurity level that results in the formation of the impurity band. The ionized impurity values obtained using Kuzmicz model [25] are in good agreement with those obtained from the model of Klassen and De Graff [23] for donor concentration upto $10^{18} \mathrm{~cm}^{-3}$ and in the temperature range from $\mathrm{T}=300 \mathrm{~K}$ to $\mathrm{T}=50 \mathrm{~K}$. Kuzmicz [25] has verified his theoretical ionization model with the experimental data obtained from the measurement of sheet resistance. He concludes that one cannot expect good agreement between resistivity calculations and experiment unless the incomplete ionization model is included. Since the model requires numerical solution of fairly 
complex equation, we have used the analytical approximation presented by Kuzmicz [25].

\section{III.2 Theoretical Modeling}

In a semiconductor, the structure of energy bands depends on the doping concentration. The changes in this structure includes formation of the band tails, impurity band formation, merging of the impurity band with the majority carrier band and change of mean impurity activation energy with doping concentration due to the rigid shifts of the majority carrier band and/or impurity band. Experimental data [33], [57], and [36] indicate that the density of quantum states in the majority carrier band in heavily doped silicon is similar in form and magnitude to the density of quantum states in this band in pure silicon. This suggests that the band tail states have insignificant effect on the carrier concentration. The Kuzmicz model [25] described below, assumes that the density of quantum states in the majority carrier band is parabolic regardless of the doping concentration. The assumption is, however not sufficient to determine the total density of quantum states of free majority carriers. In lightly doped silicon the impurity bands are localized. As the doping concentration increases, the impurity bands enter the majority carrier band and eventually merge completely with this band. Thus, in heavily doped silicon the impurity levels become delocalized. The model assumes that in n-type semiconductor all donor levels below the edge of the conduction band $\mathrm{E}_{\mathrm{c}}$ are localized, while the levels above $\mathrm{E}_{\mathrm{c}}$ are delocalized. Similarly, in p-type semiconductor all acceptor levels above the edge of the valence band $E_{V}$ are localized and the levels below $E_{V}$ are delocalized. Therefore the model is based on the following assumptions:

(a). It is assumed that in n-type silicon with total concentration of donors $N_{D}$ and no acceptor atoms, the intrinsic concentration $n_{i}$ is much less than the concentration of electrons in the conduction band. 
(b). The density of quantum states in the conduction band $\mathrm{g}_{n}(E)$ is parabolic regardless of the doping concentration.

$$
g_{n}(E)=2 \pi\left(\frac{2 m_{n}}{h^{2}}\right)\left(E-E_{c}\right)^{\frac{1}{2}} \quad \text { for } \mathrm{E}>\mathrm{E}_{\mathrm{C}}
$$

where $m_{n}$ is the effective mass and $h$ is the Planck's constant, $E_{c}$ denotes the conduction band edge.

(c). The density of quantum states in the impurity band $g_{d}$ is [39] and [49]

$$
g_{d}(E)=N_{D}\left(2 \pi \sigma_{e}^{2}\right)^{\frac{-1}{2}} \exp \left[\frac{-\left(E-E_{d}\right)}{2 \sigma_{e}^{2}}\right]
$$

where $\quad \sigma_{e}=1.036 \exp \left[-\left(11.3806 N_{D}^{+}\right)^{\frac{1}{2}} \lambda^{\frac{-3}{2}}\right.$

and the parameters $\sigma$ and $\lambda$ are defined by

$$
\sigma=\mathrm{q}\left(\mathrm{ND}^{+} / 8 \pi \varepsilon_{\mathrm{s}}^{2}\right)
$$

and $\quad \lambda=\frac{k T \varepsilon_{s}}{q^{2} N_{D}^{+}}$

where $\mathrm{ND}^{+}$denotes the concentration of ionized donors and the remaining symbols have their usual meaning. The above equations are consistent with the assumption that the total density of states in the impurity band equals the total concentration of donor atoms $\mathrm{N}_{\mathrm{D}}$

$$
N_{D}=\int g_{d}(E) d E
$$

(d). The donor activation energy is given by

$$
\Delta \mathrm{E}_{\mathrm{d}}=\mathrm{E}_{\mathrm{c}}-\mathrm{E}_{\mathrm{d}}
$$

and it is assumed that this energy decreases with doping according to $\mathrm{N}^{1 / 3}$ law

$$
\Delta E_{d}=\Delta E_{d 0}-b\left(N_{D}\right)^{\frac{1}{3}}
$$

where $b=\frac{1.32 \times 10^{18} q^{2}}{\varepsilon_{0} \varepsilon_{s}} \quad$ ev.cm 
(e). The concentration of free electrons is given by

$$
n_{0}=\int_{E_{C}}^{\infty}\left[g n(E)+g_{d}(E)\right] f(E) d E
$$

where $f(E)$ denotes the Fermi-Dirac function

$$
f(E)=\frac{1}{1+\left\{\exp \left[\frac{\left(E-E_{F}\right)}{k T}\right]\right\}}
$$

(f). The concentration of occupied localized states in the impurity band is given by

$$
n_{d}=\int_{E_{c}}^{\infty} g_{d}(E) f_{d}(E) d E
$$

where $f_{d}(E)$ is the probability function

$$
F_{d}(E)=\frac{1}{1+\left\{d_{d} \exp \left[\frac{\left(E-E_{d}\right)}{k T}\right]\right\}}
$$

and the degeneracy factor for donors $d_{d}=2$.

(g). Every donor atom introduces one donor level. Therefore, the number of occupied localized states equals the number of non-ionized donor atoms. It also follows from the neutrality condition that the concentration of free electrons equals the concentration of ionized donor atoms

$$
\mathrm{ND}^{+}=\mathrm{n}_{\mathbf{0}}
$$

Thus, the total donor concentration is given as

$$
\begin{aligned}
& \mathrm{N}_{\mathrm{D}}=\mathrm{n}_{\mathrm{o}}+\mathrm{n}_{\mathrm{d}} \\
& \mathrm{N}_{\mathrm{D}^{+}}=\mathrm{N}_{\mathrm{D}}-\mathrm{n}_{\mathrm{d}}
\end{aligned}
$$

using eqns.(48) and (52), we get

$$
N_{D}^{+}=N_{D}-\int_{\infty}^{E_{c}} g_{d}(E) f_{d}(E) d E
$$


using eqns (49) and (53),

$$
\begin{gathered}
N_{D}^{+}=N_{D} 1-\int_{\infty}^{E_{c}} \frac{g_{a u x}\left(E-E_{d}\right)}{N_{D} f_{d}\left(E-E_{d}-K T \frac{\left(E_{F}-E_{c}\right)}{K T}\right.} d E \\
=N_{D}\left[1-\int_{\infty}^{E_{c}} \frac{g_{a u x}\left(E-E_{d}\right)}{N_{D} f_{d}\left(E-E_{d}-K T x\right)} d E\right.
\end{gathered}
$$

where $g_{a u x}\left(E-E_{d}\right)$ is an auxillary function defined by

$$
g_{\text {aux }}\left(E-E_{d}\right)=\frac{g_{d}\left(E-E_{d}\right)}{N_{D}}
$$

and $x$ is an auxillary variable given by

$$
\begin{aligned}
x & =\frac{\left(E_{F}-E_{c}\right)}{k T} \\
& =N_{D}\left[1-g_{a u x}\left(E-E_{d}\right) f_{d}\left(E-E_{F}+\Delta E_{d}\right) d E\right.
\end{aligned}
$$

Also

$$
\mathrm{ND}^{+}=\mathrm{n}_{\mathbf{0}}
$$

substituting the value of $n_{0}$ from eqn (46),

$$
\begin{aligned}
\mathrm{ND}_{\mathrm{D}}+ & =\int_{E_{c}}^{\infty}\left[g_{n}(E)+g_{d}(E)\right] f(E) d E \\
& =\int_{E_{c}}^{\infty} g_{n}(E) f(E) d E+\int_{E_{c}}^{\infty} g_{d}(E) f(E) d E
\end{aligned}
$$

Density of states in the conduction band

$$
g_{n}(E)=2 \times 2 \pi\left(\frac{2 m_{n}^{*}}{h^{2}}\right)\left(E-E_{c}\right)^{\frac{1}{2}}
$$


Define a dimensionless variable,

$$
y=\frac{\left(E-E_{c}\right)}{k T} \text { and } z=\frac{\left(E-E_{F}\right)}{k T}
$$

Therefore,

$$
\begin{gathered}
\int_{E_{c}}^{\infty} g_{n}(E) f(E) d E=2 \times 2 \pi\left(\frac{2 m_{n}^{*}}{h^{2}}\right)^{\frac{3}{2}} \int_{E_{C}}^{\infty} \frac{\left(E-E_{C}\right)^{1 / 2}}{\left(1+\exp \frac{\left(E-E_{F}\right)}{K T}\right]} d E \\
=2 x 2 \pi\left(\frac{2 m_{n}^{*}}{h^{2}}\right)^{\frac{3}{2}}(k T)^{\frac{3}{2}} \int_{0}^{\infty} \frac{y^{1 / 2}}{(1+\exp (y-x)} d y
\end{gathered}
$$

Fermi-Dirac integral of order $1 / 2$ is

$$
\begin{aligned}
& \mathrm{F}_{1 / 2}(\mathrm{x})=\frac{2}{\pi} \int_{0}^{\infty} \frac{y^{1 / 2}}{(1+\exp (y-x)} d y \\
& \int_{E_{C}}^{\infty} g_{n}(E) f(E) d E=2\left(\frac{2 \pi k T m_{n}^{*}}{h^{2}}\right)^{\frac{3}{2}} \mathrm{~F}_{1 / 2}(\mathrm{x})
\end{aligned}
$$

But $2\left(\frac{2 \pi k T m_{n}^{*}}{h^{2}}\right)^{\frac{3}{2}}=\mathrm{N}_{\mathrm{c}}$

$$
\int_{E_{c}}^{\infty} g_{d}(E) f(E) d E=\int_{E_{c}}^{\infty} g_{a u x}\left(E-E_{d}\right) f_{d}\left(E-E_{F}+\Delta E_{F}\right) d E
$$

Subsituting eqns.(64) in eqn (59), we get 


$$
\begin{aligned}
& \mathrm{ND}^{+}=\mathrm{N}_{\mathrm{c}} \mathrm{F}_{1 / 2}(\mathrm{x})+\mathrm{N} \mathrm{D} \int_{E_{c}}^{\infty} g_{\text {aux }}\left(E-E_{d}\right) f\left(E-E_{c}-K T x\right) d E \\
& \mathrm{ND}^{+}=\mathrm{N}_{\mathrm{c}} \mathrm{F}_{1 / 2}(\mathrm{x})+\mathrm{N}_{\mathrm{D}} \int_{E_{c}}^{\infty} g_{a u x}\left(E-E_{d}\right) f\left(E-E_{c}-\Delta E_{d}\right) d E
\end{aligned}
$$

where $g_{d}\left(E-E_{d}\right)$ is determined by eqns. (38)-(41). The system (60)-(68) may be solved numerically for $\mathrm{x}$ and $\mathrm{N}_{\mathrm{D}}{ }^{+}$provided that the total donor concentration $\mathrm{N}_{\mathrm{D}}$ is given. Solving the system (60)-(68) for a set of values of $N_{D}$ we obtain the concentration of ionized impurity carriers as a function of the total doping concentration.

The theoretical model of ionization of impurity atoms for p-type silicon is based on a similar set of assumptions. The only exception is the degeneracy factor in eq. (49).

$$
d_{a}=4+2 \exp \left(E-E_{d} / K T\right)
$$

where $\mathrm{d}=44 \mathrm{meV}$

To facilitate the calculation of the fraction of ionized dopants, given below is an analytical approximation [25] which was used in the original simulator [5] to model the ionized impurity concentration..

$$
\frac{N_{D}^{+}}{N_{D}}=1-A \exp \left\{-\left(B \ln \left[\frac{N}{N_{o}}\right]\right)^{2}\right\}
$$

Where A, B and No are constants and are determined as follows:

For n-type silicon,

$$
\begin{aligned}
\mathrm{A} & =0.0824 \mathrm{~T}_{\mathrm{n}}-1.622 \\
\mathrm{~N}_{\mathrm{O}} & =1.6 \times 10^{18} \mathrm{~T}_{\mathrm{n}} 0.7267 \\
\mathrm{~B} & =0.4722 \mathrm{~T}_{\mathrm{n}} 0.0652 \quad \text { for } \mathrm{N}<\mathrm{N}_{\mathrm{O}} \\
& =1.23-0.3162 \mathrm{~T}_{\mathrm{n}} \quad \text { otherwise } \quad \text { Where }_{\mathrm{n}}=\frac{T}{300}
\end{aligned}
$$

For p-type silicon, 


$$
\begin{aligned}
A & =0.2364 \mathrm{~T}_{n}-1.474 \\
\mathrm{~N}_{0} & =1.577 \times 10^{18} \mathrm{~T}_{n} 0.46 \\
\mathrm{~B} & =0.433 \mathrm{~T}_{\mathrm{n}} 0.2213 \text { for } \mathrm{N}<\mathrm{N}_{\mathrm{o}} \\
& =1.268-0.338 \mathrm{~T}_{\mathrm{n}} \quad \text { otherwise }
\end{aligned}
$$

Where $\mathrm{T}_{\mathrm{n}}=\frac{T}{300}$

The above approximation has been evaluated at room temperature and at low temperature and then included in the simulator. Fig 6 shows the inclusion of the new ionization model into the simulator. The inital value of the ionized donor and acceptor concentrations are calculated using the original ionization model described in equation (31) and (32) in Chapter II along with the other physical parameters. After obtaining the initial guess file, the physical parameters are again updated. The flowchart of the simulator given in Fig 3 demonstrates this clearly. The recalculation of the ionized donor and acceptor impurity atoms is done after calculating the potential distribution and the Quasi-Fermi exponentials.

\section{III.3 Results And Discussions}

The results of the calculation using the analytical approximation based on Kuzmicz model [25] is shown in Fig 7 as a function of temperature and impurity concentration. From this figure it can be seen that complete ionization of impurities is achieved at concentrations greater than $10^{19} \mathrm{~cm}^{-3}$. Comparing these results with those of Klassen and de Graff [23] in Fig 5, we see that even though the two plots show the same nature, complete ionization of impurity atoms is achieved by the Klassen et al [17] model at $N_{d}$ $>10^{18} \mathrm{~cm}^{-3}$. For values of donor concentration less than $10^{18} \mathrm{~cm}^{-3}$ there is a good agreement between the two models [23], [25]. However, the model of Klassen et al [23] 


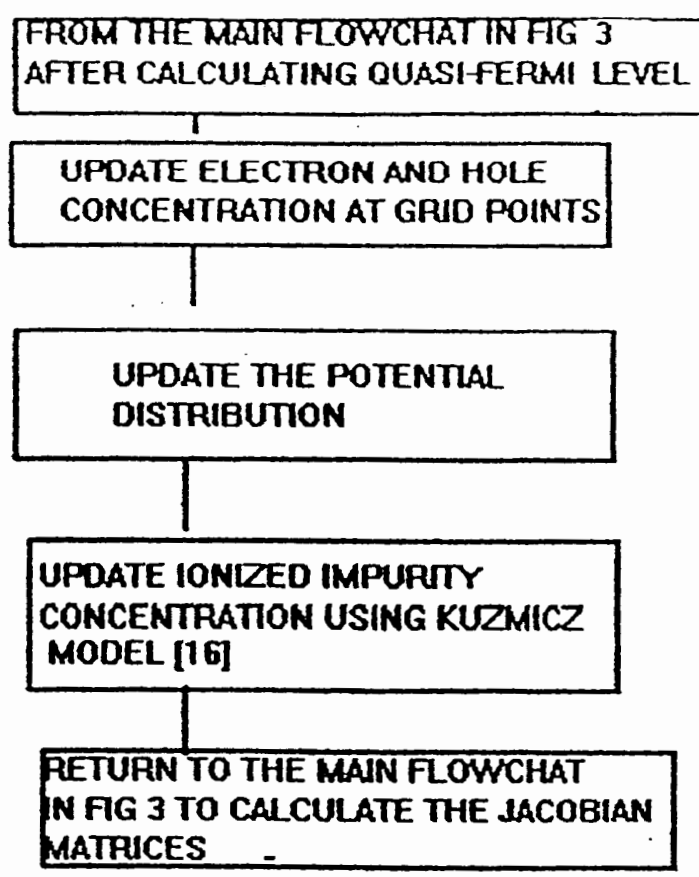

Figure 6. Incomplete ionization model flowchart

shows a discontinuity at high concentrations. The model [23] has been used largely for MOS device modeling and BJT modeling were the concentration are low.

The Kuzmicz model was introduced in the new C-version of BiLow to model Mott transition and incomplete ionization and the continuity of the model was verified by comparing the net ionized impurity distribution at $\mathrm{T}=77 \mathrm{~K}$ and $\mathrm{T}=300 \mathrm{~K}$ as obtained from the simulation results using the original and new version of BiLow. The net ionized impurity distribution at $\mathrm{T}=77 \mathrm{~K}$ and at $\mathrm{T}=300 \mathrm{~K}$ for low level injection for the original BiLow is shown in Fig 1 and from the new version is shown in Fig 8. In both the figures one can see that the donor concentration in most of the emitter is above the Mott transition so the ionized donor concentration is not affected by freezeout. The concentration of acceptors in the base region and the concentration of donors in the collector region are both below the Mott transition, so they are temperature dependent and suffer from freezeout. In Fig 4 one can see the abrupt decrease in the electron and 


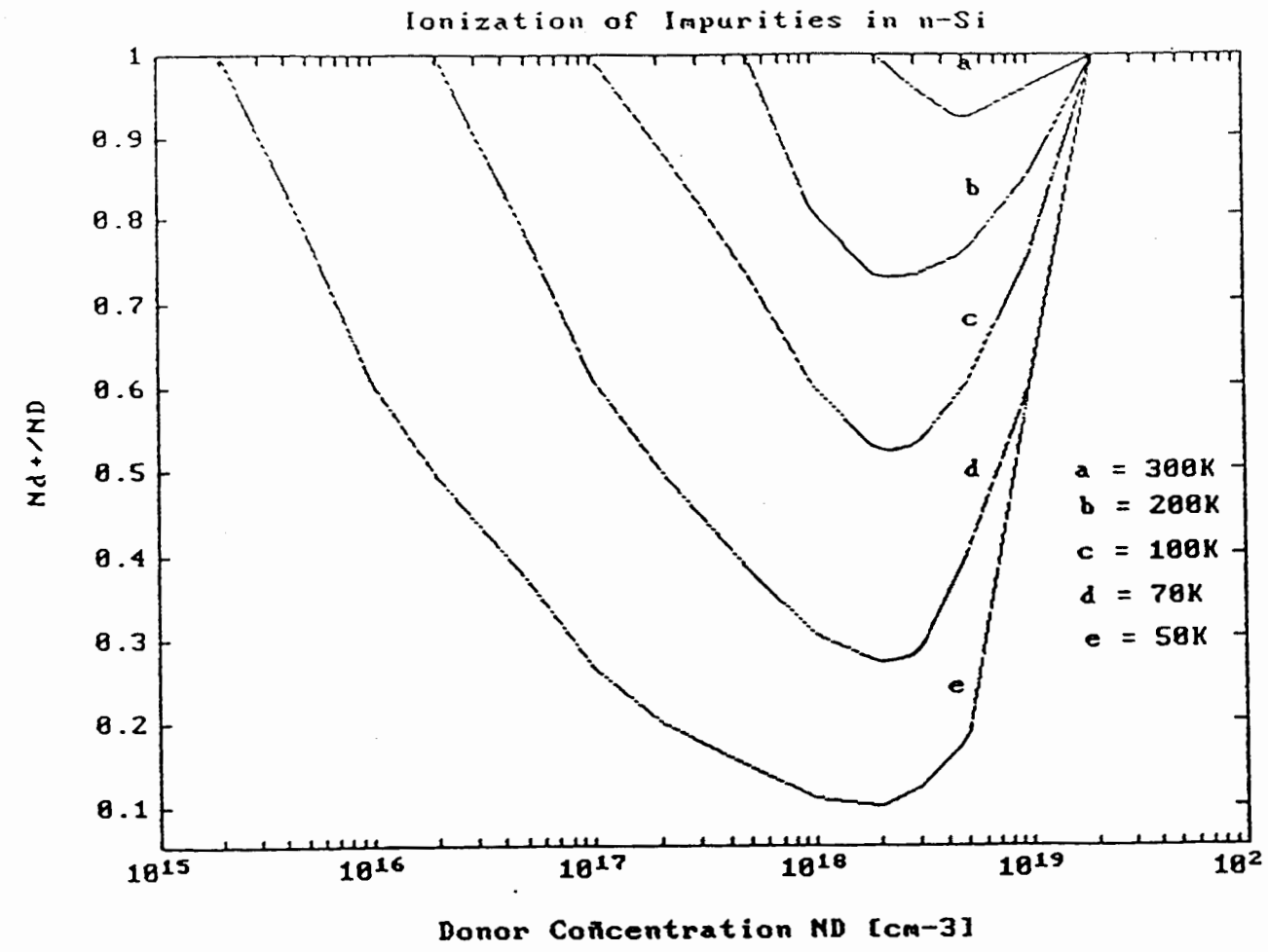

Figure 7. Ratio of the concentration of ionized impurity to the total doping concentration as a function of temperature and doping

concentration in the emitter at $77 \mathrm{~K}$. This is mainly due to the fact that there is a discontinuity in the Mott transition model at concentration around $3 \times 10^{18} \mathrm{~cm}^{-3}$. This discontinuity is removed in the new BiLow by using the model described in section III.2. In Fig 8 the doping profile has smoothen out at $\mathrm{T}=77 \mathrm{~K}$ with a continuity through the Mott transition region. 


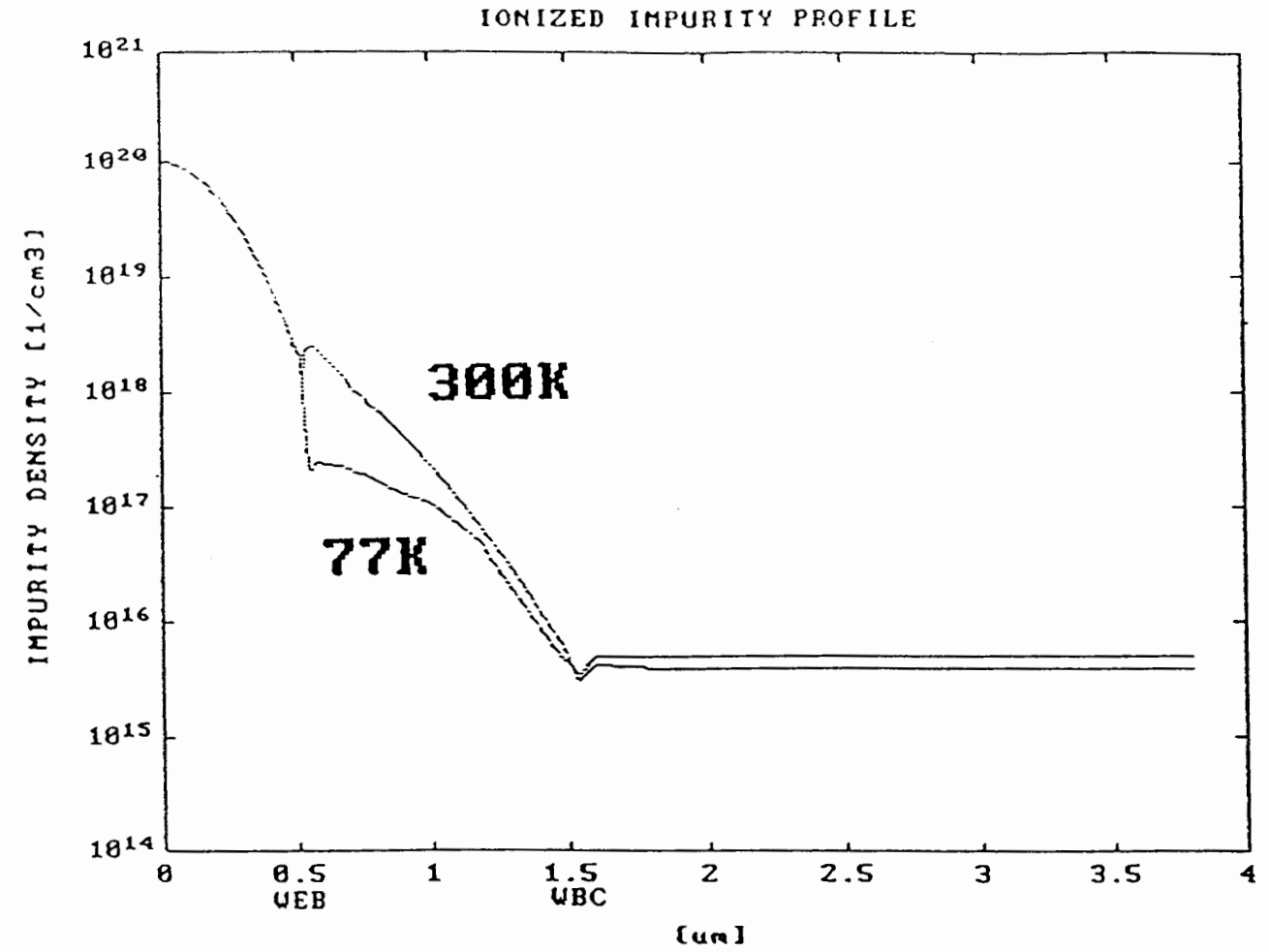

Figure 8. Total ionized dopant distribution in the transistor at $\mathrm{T}=300 \mathrm{~K}$ and $\mathrm{T}=77 \mathrm{~K}$ using the new BiLow 
CHAPTER IV

\section{BANDGAP NARROWING IN SILICON}

\section{IV.1 Background}

Heavily doped regions are present in every semiconductor device of today. In addition to this, a reduction in the vertical dimensions for VLSI devices is also imperative [59]. The need to provide these thin, low resistive layers as well as tightly controlled depletion regions pushes the overall doping throughout the device to even higher levels. Because of its practical importance, extensive studies on heavily doped Si have been going on for a long time. Bandgap narrowing (BGN) which is the shrinkage of the energy gap at high doping concentrations is one parameter that has gained importance. The concentration of dopant impurities affect both the density of states associated with the host lattice as well as the density of states associated with the impurity atoms [60]. In heavily doped silicon, the energy band structure changes due to many body effect which results in the broadening of the impurity band and the band tail effect that results from the randomness of the impurity distribution on the edge of the conduction band and the valence band [60]. These effects impact the energy gap in the emitter and the base region. An increase in the bandgap narrowing, results in lower emitter efficiency and an increasing temperature dependence of the current gain [31]. However, the need to design higher performance devices requires a better understanding of the minority-carrier transport parameter in heavily doped silicon. Slotboom and De Graff [48] were among the first to fabricate devices designed to measure the influence of high doping on device performance. Following their work, a number of researchers have carried out both 
experimental as well as theoretical work on what has become to be called "device bandgap narrowing", "apparant bandgap narrowing " or, simply "bandgap narrowing". In the following section we have tried to analyze the available literature on BGN in order to come up with a good model which is valid in the regions of low and high doping.

\section{IV.2 Previous Work}

The use of the name " bandgap narrowing " for denoting the parameter $\Delta E_{g}$ in device simulations is very unfortunate. Device measurements give the pn product in equilibrium, at most [34], [40], [51], and [55]. The extraction of the value of the semiconductor bandgap from the measurement of $n_{i e}{ }^{2}$ requires a theoretical model and several assumptions regarding the density of states equation [9], for example, requires that Maxwell-Boltzman statistics are applicable, and that the density of states in the conduction and valence band be parabolic. The first assumption is clearly invalid. The other has not been proven. Therefore, it should not be surprising, that $\Delta \mathrm{E}_{\mathrm{g}}$ as obtained from a theoretical model and the resulting value of $\Delta E_{g}$ from optical and photoluminescence measurements are drastically different. Fig 9 presents the discrepancies of the BGN data, which were obtained from the electrical measurements of Slotboom et al [48], Weider [58], Mertens et al [33], and Nugroschel et al [36], and from the luminescence measurements of Dumke [13]. Additionally, part of the bandgap extraction problem lies in the way various workers have extracted $n_{i e}{ }^{2}$ from $I-V$ measurements, making assumptions that are not based on experimental observations. We have, therefore, grouped the literature survey into two, Group1 deals with the optical and electrical measurements and the Group2 deals with the theoertical derivations of BGN.

Groupl Experimental (Optical and Electrical Measurements): Most of the discrepancies in BGN values arise from the differences in the assumptions about mobility and, in some cases, from the lifetime data used in the interpretation of the experimental 
data. During the last few years a number of experimental results have been published which show that, starting at a doping concentration of $10^{18} \mathrm{~cm}^{-3}$, minority carrier mobility in silicon exceeds majority carrier mobility upto a factor of three at a concentration of $10^{20} \mathrm{~cm}^{-3}[24],[35]$ and [56].

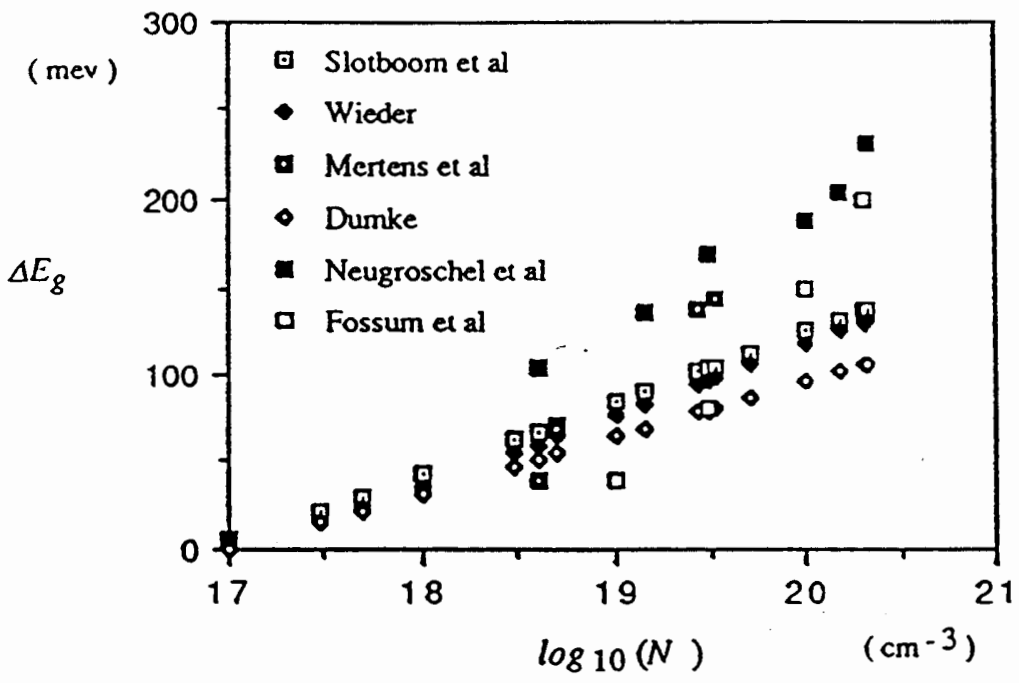

Figure 9. Summary of bandgap narrowing from different measurements. $\mathrm{N}=\left[10^{17}, 2.1 \times 10^{20}\right] \mathrm{cm}^{-3}[9]$

Theoretical calculations have shown a long time ago that minority carrier mobility may exceed majority carrier mobility also at low-temperature [30] or at higher doping [41]. However, this did not result in the formulation of the mobility model that can be used in device simulation. Klassen [24] has presented an analytical model that unifies the description of majority carrier and minority carrier molvility and includes screening of the impurities by charge carriers, electron-hole srattering, clustering $\mathrm{c} f$ impurities and the full temperature dependence of both majority and minority carrier mobilities. The excellent agreement between this model and published experimental data on the carrier mobility in silicon [56] ensures that this model is a sound basis for the revised 
determination of bandgap narrowing work. The optical and experimental works on BGN are described separately in the following section.

Electrical Measurements: Klassen, Slotboom and De Graff [23] have used the Klassen mobility model [24] to recalculate the bandgap narrowing obtained from the experimental measurements upon which the most of the BGN models [48], [8]-[10] are based. The new bandgap narrowing value is given as the sum of the reported BGN value using the temperature independent $\Delta \mathrm{V}_{\mathrm{go}}(\mathrm{N})$ [48] plus the correction which includes the temperature dependence of mobility. The relationship between the new(corrected) value for the bandgap narrowing $\Delta V_{\text {go }}{ }^{\text {new }}$ and the reported value $\Delta V_{\text {go }}{ }^{\text {rep }}$ is found to be[23]

$$
\Delta \mathrm{V}_{\mathrm{go}} \text { new }=\Delta \mathrm{V}_{\mathrm{go}} \text { rep }+\frac{k T}{q} \ln \left(\frac{\mu^{\text {rep }}}{\mu^{\text {new }}}\right)+\frac{k T}{q} \ln \left(\frac{C_{1}^{\text {rep }}}{C_{2}^{\text {new }}}\right)
$$

The parameters used by different authors can be found in Table I [23]. Figure 9 shows the apparant bandgap narrowing as a function of impurity concentration. In the upper part, the new BGN value calculated from the Klassen et al. method [23] for p-type Si are smaller due to the minority electron mobility which is larger than the majority carrier mobility. Also for the data obtained by Slotboom et al. [48] with eqn.(71), the new value of BGN is smaller due to the difference in the temperature dependence between minority and majority carrier mobilities. Compared to the data reported by Del Alamo et al. [9]

Table I

Model parameters used in the interpretation ofexperimental data

\begin{tabular}{lllcc} 
& $\begin{array}{l}\text { Slotboom and de Graff } \\
\text { (p-type) [48] }\end{array}$ & $\begin{array}{c}\text { Del Alamo et al } \\
\text { (n-type) [9] }\end{array}$ & $\begin{array}{c}\text { Swirhun et al. } \\
\text { (p-type) }[49]\end{array}$ & $\begin{array}{c}\text { Klassen et al. } \\
\text { (n-p-type) [23] }\end{array}$ \\
\hline $\mathrm{C}_{1}\left(\mathrm{~cm}^{-6} \mathrm{~K}^{-3}\right)$ & $9.61 \times 10^{32}$ & $1.38 \times 10^{33}$ & $1.26 \times 10^{33}$ & $9.61 \times 10^{32}$ \\
$\mathrm{~V}_{\mathrm{g}}(\mathrm{mV})$ & 1.206 & 1.206 & 1.206 & 1.206 \\
$\mathrm{C}_{2}$ & 0.5 & 0 & 0.5 & 0.5 \\
\hline
\end{tabular}


for $\mathrm{n}$-type $\mathrm{Si}$, the $\mathrm{BGN}$ values are larger. This is due to the difference in parameter $\mathrm{C}_{1}$ for the intrinsic concentration at high concnetration while it is due to the difference in the minority hole mobility and is clearly visible in the first row of the Table I for $\mathrm{n}$ and p-Si.

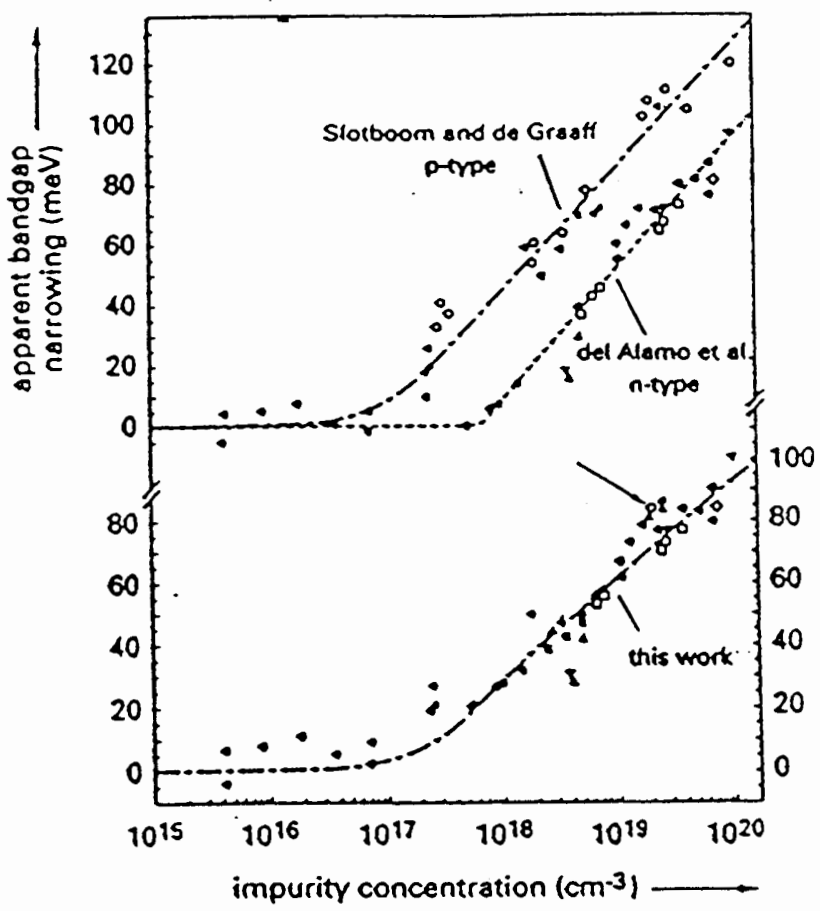

Figure 10. Bandgap narrowing as a function of impurity concentration [23]

In the lower part of Fig 10 the corrected value of BGN in both n- and p-type Si are given as a function of impurity concentration. The conclusion of Klassen et al. [23] was that all the new BGN values for $n$ - and p-type Si lie close together and moreover, the bandgap narrowing can be shown to be temperature independent between $280-400 \mathrm{~K}$. The existing BGN model in the original BiLow [5] follows the Slotboom et al [48] model at room temperature. As such we have not taken the Klassen et al model [23], which is the same as Slotboom model plus a correction, to be included in the simulator. Swirhun et al [49] have measured the electron diffusion length and electron lifetime to extract the minority 
carrier electron mobility as a function of doping density and concluded that the electron mobility is about 2.5 times larger in heavily doped p-type Si than in n-type. Using the Slotboom et al. model [48] they found $25 \mathrm{meV}$ more bandgap narrowing in p-Si than in equivalently doped $n-S i$. In their work of qualifying $\Delta E g$ on $N_{A}$ experimentally, King and Swanson [21] have attributed the various discrepancies on the assumption that minority carrier mobility equals majority carrier mobility and the value of $n_{i o}$ at $300 \mathrm{~K}$ used by different authors in extracting this parameter. Weybright and Plummer [57] have done a comparison of simulated Gummel plots with different BGN models. They concluded that King's BGN model [21] for p-type material gives the most satisfactory agreement over temperature, Swirhun's [49] and Slotboom's [48] models overestimate the collector current over temperature, but the Del Alamo's BGN model [10] for $n$ - and p-type material gives the better fit to the measured data. Given in Table II are the BGN model parameters as used by Del Alamo [10], King [21], Slotboom [48] and Swirhun [49].

TABLE II

Comparison of BGN model parameters

BGN(effective $)=k I n[N / N c)$

\begin{tabular}{|c|c|c|}
\hline Model & $\mathrm{K}$ [meV] & $N c[c m-3]$ \\
\hline del Alamo & 18.7 & $1.61 \mathrm{e} 18$ \\
\hline King & 17.8 & $2.3 \mathrm{e} 17$ \\
\hline Slatboom & 18.1 & $4.23 e 16$ \\
\hline Swirhun & 7.38 & $3.03 \mathrm{e} 16$ \\
\hline
\end{tabular}


Kuzmicz and Wosik [27] performed steady state measurements on bipolar test structure and came up with a new approximation for the bandgap narrowing and minority carrier mobility in silicon, valid at all doping levels.

$$
\Delta \mathrm{Eg}=a \mathrm{n}_{\mathrm{m}}{ }^{1 / 3}
$$

where $a$ is an emperical parameter calculated from the theoretical values of collector current and taken to be equal to $3.6 \times 10^{-8} \mathrm{eV}-\mathrm{cm}$ and $\mathrm{n}_{\mathrm{m}}$ is the majority carrier concentration. All measurements were performed at 300K. Kuzmicz and Wosik [27] then calculated the diffusion length from the bandgap and lifetime approximations proposed by them as well as used those proposed by Del Alamo [9]. The agreement was good for doping concentration below $10^{19} \mathrm{~cm}^{-3}$ but the difference increases quickly with doping. Based on other experimental comparison between their results and those of Del Alamo [9], Kuzmicz and Wosik [27] believe that their approximation agrees better with the experiment than do those of Del Alamo [9]. Our aim has been to study the different BGN models and to chose a model which is continuous over a wide range of donor concentration and can also be extented to low-temperature. The Kuzmicz and Wosik model claims to be valid for medium and high doping and could be extended to lower temepratures. We have therefore added this model in the simulator.

Optical Measurements: Wagner and Del Alamo [55] have compiled a set of Photoluminescence (PL) data [52]-[54] for the bandgap narrowing in n-type and p-type silicon at low temperature $(20 \mathrm{~K})$ and at room temperature $(300 \mathrm{~K})$. The room temperature PL data obtained from n-type material are compared to electrical data [9] for bandgap narrowing in heavily doped $\mathrm{n}-\mathrm{Si}$. The PL data are found to be fully consistent with low temperature selective absorption Photoluminescence excitation (PLE) measurements, which also cover $n$ - and p-type material upto charge carrier concentration of $\sim 10^{20} \mathrm{~cm}^{-3}$. The optical bandgap energy (due to indirect transition) remains almost constant over the whole range of concentration $\left(4 \times 10^{18}-4 \times 10^{20} \mathrm{~cm}^{-3}\right)$ except for most heavily doped $\mathrm{p}$ - 
type boron samples. This indicates that for the present range of carrier concentrations the band filling is almost canceled by the bandgap narrowing [7]. For the $300 \mathrm{~K}$ spectra the direct extraction of optical bandgap is not possible due to the thermal spread of the carriers which smears out the high energy cutoff. Thus, Wagner and Del Alamo [55] calculated the optical bandgap at $300 \mathrm{~K}$ from the $20 \mathrm{~K}$ temperature data. A value of $60 \mathrm{meV}$ was added to the bandgap energy values of $20 \mathrm{~K}$ to get the bandgap energy value at $300 \mathrm{~K}$, assuming that the temperature shift of the bandgap is independent of the doping level [8]. Within the experimental accuracy of $+10 \mathrm{meV}$, the same BGN was found at $20 \mathrm{~K}$ and at $300 \mathrm{~K}$ [53]. Fig1 1 and Fig12 display $\Delta \mathrm{Eg}$ as a function of carrier concentration at $20 \mathrm{~K}$ and $300 \mathrm{~K}$.

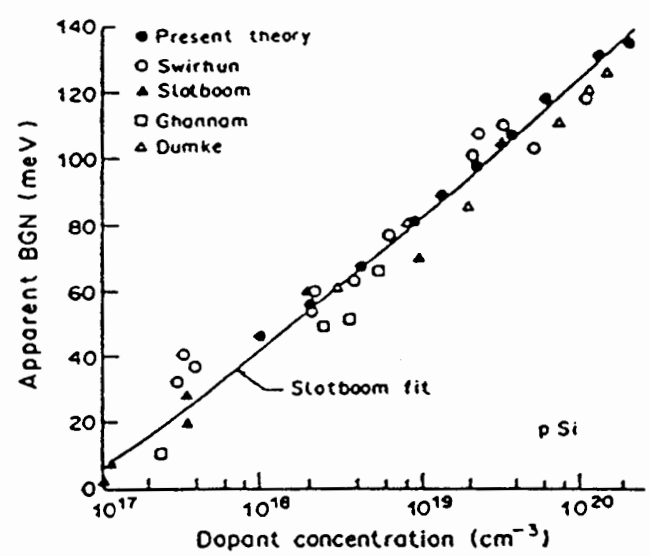

Figure $11 . \Delta$ Eg vs carrier concentration at $20 \mathrm{~K}$ [55]

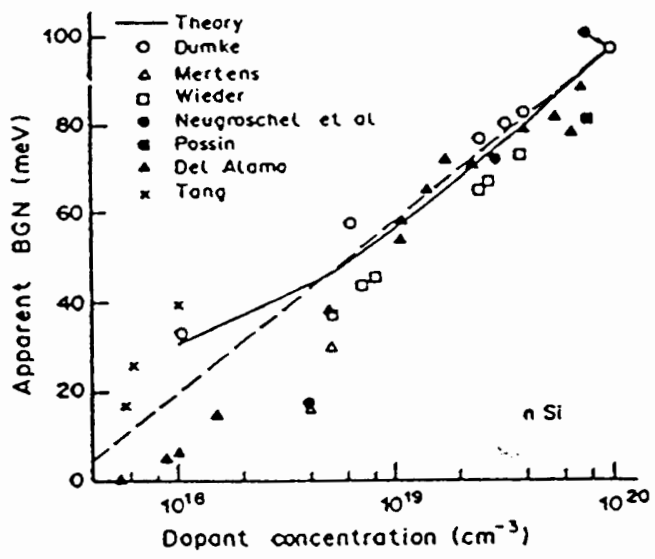

Figure 12. $\Delta \mathrm{Eg}$ vs carrier concnetration at $300 \mathrm{~K}$ [55]

In the plots, the data reported by Dumke[13] was also compared. This data [13] was deduced from a line shape analysis. The data by Dumke [13] and the data of Wagner and Del Alamo [55] are in good agreement with each other. The data for BGN derived from 
PL measurements were confirmed by selective absorption spectra recorded at low temperature using PLE spectroscopy. Based on the experimental data of [55] as well as by reinterpreting a number of data published in the literature [33], [57] Del Alamo, Swirhun and Swanson derived a consistent set of electrical data for bandgap narrowing in heavily doped n-type silicon [10].

$$
\begin{aligned}
\Delta \mathrm{E}_{\mathrm{g}} & =18.7 \times 10^{-3} \ln \left(\frac{N_{D}}{7 \times 10^{17}} \mathrm{~cm}^{-3}\right)[\mathrm{eV}] & & \text { for } \mathrm{ND}_{\mathrm{D}}>10^{17} \mathrm{~cm}-3 \\
& =0 & & \text { otherwise }
\end{aligned}
$$

Boltzman statistics was used for the whole range of carrier concentrations. The agreement between the electrical [9] and the optical values [55] for the bandgap narrowing was surprisingly good considering the fact that completely different experimental techniques have been used. This implied that the bandgap narrowing measured by optical technique [55] was the same as the bandgap narrowing relevant to device properties. Both optical [55] and electrical data [9] were derived assuming parabolic bands for the majority and minority carriers. However band tailing of minority carrier band can affect the PL data for higher concentrations. Also the electrical data is expected to be sensitive to band tail states. Nevertheless, data obtained from both the techniques are in good agreement of each other. This suggests, either, that the density of states in the valence band tail is rather small or that the tails affect the electrical and the optical measurements in the same way. Previous efforts to correlate theoretical and experimental data are incomplete for a number of reasons [13]. These efforts were based on low-temperature PL data which were scaled upto room temperature. For n-type material with carrier concentration exceeding $10^{18} \mathrm{~cm}^{-3}$, these scaled PL data gave a smaller bandgap narrowing [13] than the electrical data of Wieder [58] and Tang [51]. For p-type material a better agreement was found between the PL data and the transport data of Slotboom and de Graff [48]. However, the PL data in that case was not obtained 
from a complete line shape analysis. They were deduced from the high energy cut off in the low temperature PL spectrum by subtracting calculated values for the band filling $E_{\mathrm{V}^{-}}$ $E_{\mathbf{f}}$

From the optical [55] and experimental [48], [9], [49], [23] measurements, it can be concluded that the bandgap narrowing in $\mathrm{n}$-and $\mathrm{p}-\mathrm{Si}$ is the same. These measurements have also shown that $B G N$ is independent of temperature.

Group2 (Theoretical Expressions): On the theoretical side, Mahan [32] using the many-body technique and Berggren and Sernelius [46] using the second order perturbation theory calculated the shift in the bandgap due to heavy doping. Mahans approach was elegant in that he used the many -body technique and still obtained a very simple expression for the BGN. However, since Mahan assumed the impurity atoms to be distributed in a periodic lattice in $\mathrm{Si}$ and $\mathrm{Ge}$, his results for bandgap shift due to carrier impurity interaction were in error. The results of the theory of Berggren and Sernelius [46] are in reasonable agreement with the experimental results of Wagner and del Alamo [55] in the high density region where the theory is applicable. Since the theory of Mahan and Berggren et al. does not apply at high doping, we have not considered those models.

In previous years, calculations and experiments have been made which have shown that heavy doping effects strongly influence charge transport process in bipolar semiconductor device and that these effects must be taken into account for the modeling of the present-day structure. Therefore calculation of effective bandgap narrowing is important for electron and hole mobilities and densities in heavily doped $\mathrm{Si}$. The calculations also show that effective bandgap narrowing is much larger in heavily doped and compensated than in uncompensated Si [33], [34] and [29]. Polsky and Rimshans [40] used a semiclassical approximation for the determination of effective bandgap narrowing in compensated cases. The Polsky BGN model was extracted using the linear screening theory of charges for doping concentration greater than $10^{23} \mathrm{~cm}^{-3}$ and for the 
temperature range of $\mathrm{T}=300-900 \mathrm{~K}$. They concluded that the results of their calculations agreed sufficiently well with the measured data [40]. The Polskys BGN model was the only model that we found in the literature survey which talks about BGN in compensated material. A short description is provided above just for record. The Ploskys model [40] equation is complicated and cannot be directly used for simulation.

In their work, Jain and Roulston [20], discuss bandgap narrowing in high density regime (dopant concentration $>1 \times 10^{18} \mathrm{~cm}^{-3}$ ) in $\mathrm{n}$ - and p-type Si and come up with an analytical expression for BGN as a function of majority carrier concentration at room temperature.

For $\mathrm{n}-\mathrm{Si}$,

$$
\Delta \mathrm{E}_{\mathbf{g}}=10.23\left(\frac{N}{10^{18}}\right)^{\frac{1}{3}}+13.12\left(\frac{N}{10^{18}}\right)^{\frac{1}{4}}+2.93\left(\frac{N}{10^{18}}\right)^{\frac{1}{2}}
$$

For $\mathrm{p}-\mathrm{Si}$,

$$
\Delta \mathrm{E}_{\mathrm{g}}=11.07\left(\frac{N}{10^{18}}\right)^{\frac{1}{3}}+15.17\left(\frac{N}{10^{18}}\right)^{\frac{1}{4}}+5.07\left(\frac{N}{10^{18}}\right)^{\frac{1}{2}}
$$

The semiconductor is assumed to be uncompensated and all impurities ionized so that $\mathrm{N}$ is also the concentration of free carriers. Values of bandgap narrowing using eqns (74)(75) were found to be larger in $\mathrm{p}-\mathrm{Si}$ than those in $\mathrm{n}-\mathrm{Si}$ throughout the whole range of impurity concentration. Several researchers have attempted to determine the difference in BGN at room and at low temperatures. Since calculations at room temperature are complicated and the difference between room and low temperature BGN is small, reliable values of the temperature correction cannot be obtained. The high density theorey used by Jain et al [20] to extract $B G N$ at $T=300 \mathrm{~K}$, is valid at low temperature. Therefore to extend their BGN model to low temerature, Jain et al. [20] have used a value between 0 and $20 \mathrm{meV}$ for this temperature correction [29], [54] and adopted the following procedure to do this. After applying the Femi-Dirac correction to the bandgap 
narrowing, Jain et al. [20] have added a small constant temperature correction up to $20 \mathrm{meV}$ to the calculated values of $B G N$ so as to bring their $B G N$ results in agreement with the experimental value [13], [33], [36], [39], [49] and [57]. For p-Si, Jain et al [20] have added a temperature correction of $17 \mathrm{meV}$. The results of the theory for $\mathrm{p}$-Si with a temperature correction of $17 \mathrm{meV}$ are in good agreement with all experimental data and in almost exact agreement with the Slotboom fit extended by Dumke[13] and Del Alamo et al. [9] to higher concentration. The case of $\mathrm{n}-\mathrm{Si}$ is less satisfactory. The optimum temperature correction is rather small, about $5 \mathrm{meV}$. The spread in the experimental data points is much larger than in case of p-Si. At low concentration it is as much as $30 \mathrm{meV}$. Fig 13 and Fig 14 show the calculated and the experimental BGN reported by Jain et al. [20] as a function of doping concentration.

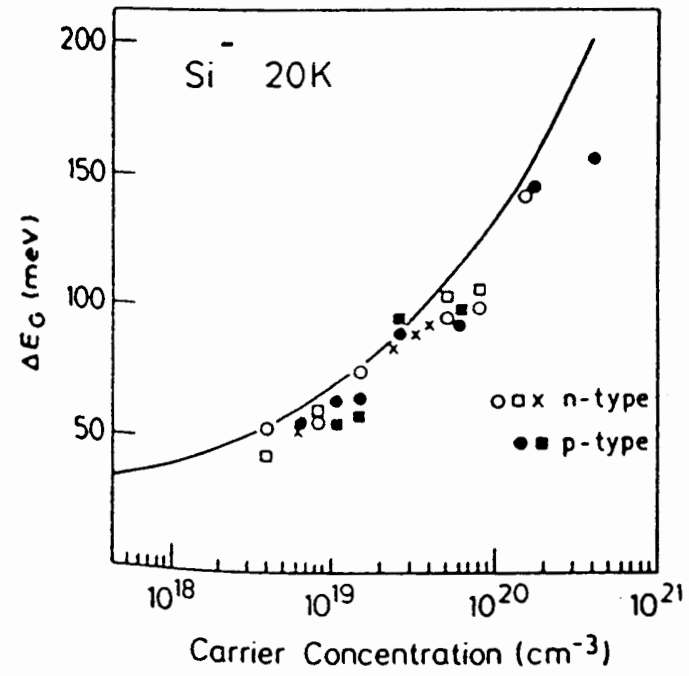

Figure 13. BGN values in $\mathrm{p}-\mathrm{Si}[20]$

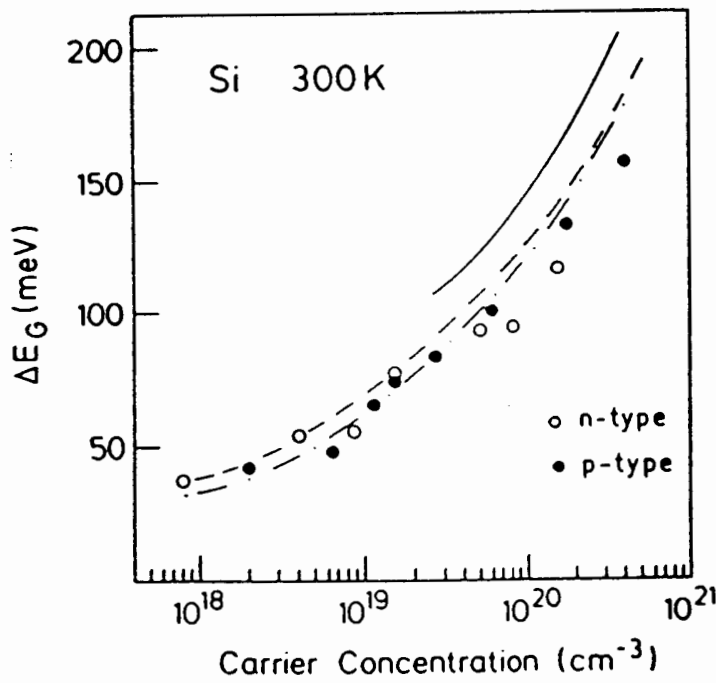

Figure 14. BGN values in n-Si [20]

Lanyon and Tuft [28] developed a theory of bandgap narrowing which takes into account the electrostatic energy of interaction between a minority carrier and a majority carrier surrounding it. This energy reduces the thermal energy required to create an 
electron-hole pair. In contrast to the preceding theories which calculated the effects of high doping on individual conduction and valence bands, the Lanyon and Tuft [28] theory calculates a pair energy similar to the excitonic binding energy of bound electronhole pairs in insulators. They calculate the pair energy and equate it to the bandgap reduction, thus reducing its activation energy.

$$
\Delta \mathrm{E}_{\mathrm{g}}=\frac{3 q^{2}}{16 \pi \varepsilon a_{s}}
$$

where $\mathrm{a}_{\mathrm{S}}$ is the screening radius and is given by $\mathrm{a}_{\mathrm{S}}=\left(\frac{q^{2} n}{\varepsilon k T}\right)^{\frac{-1}{2}}$

They concluded that at low doping levels, the temperature dependence of BGN and the square root dependence on the doping level can be seen. At higher doping levels above $10^{20} \mathrm{~cm}^{-3}$ the temperature independent $\mathrm{n}^{1 / 6}$ dependence is followed. Comparing experimental data with theory, Lanyon and Tuft [28] have excluded all data that assumed in their interpretation that bandgap narrowing is independent of temperature except those of Neugroschel et al.[36] which are in the degenerate region $10^{20} \mathrm{~cm}^{-3}$. The agreement between various workers is quite good on the whole. There are no adjustable parameters and there is no difference in the narrowing for $\mathbf{n}$ and $\mathrm{p}$-type samples.

From Group 1, Wagner et al [55] and Klassen et al [23] along with other experimental works [9], [10], [48], [49] conclude that the bandgap narrowing in $\mathrm{n}-\mathrm{Si}$ and $\mathrm{p}-\mathrm{Si}$ is identical and is independent of temperature. From the theoretical group, Jain et al [20] have found that bandgap narrowing in $\mathrm{p}-\mathrm{Si}$ is larger than that in $\mathrm{n}-\mathrm{Si}$ for the entire range of impurity concentration. They also predict a possible temperature dependence of BGN and add a temperature correction to the BGN expression in order to obtain agreement at high concentration between their calculations and the uncorrected data at room temperature. 


\section{3 Method Used In This Study}

Experimental determination of BGN over temperature is limited and theoretical calculations have not been experimentally verified. This can be seen in the literature survey presented in Section IV.2. Surveying the BGN models described in group1 and group2, we selected two different models to be added to the simulator. The Del Alamo's BGN model [10] and the Kuzmicz model [27]. From Weybright and Plummer's [57] model comparison, it was found that Del Alamo's model gave a better fit to the measurement results for both n-and p-type materials at high doping concentrations. Besides the Del Alamo model is backed by PL work of Wagner et al. [55]. The model of Jain et al. [20] was neglected as it showed a lot of discrepancies between theory and experiment for p-Si. However, the claim of Kurmicz and Wosik [27] that their approximation of BGN is valid for all doping levels was interesting enough to be considered as our goal was to study the effectiveness of the models for low, moderate and high doping concentrations. The original BGN model in BiLow [5] is similar to the Slotboom [48] BGN model at room temperture. However, at $77 \mathrm{~K}$, there is a non negligible difference between the two models due to incomplete ionization [23]. In the original model, the doping concentration at room temperature has been combined with the BGN temperature dependence from Lanyon and Tuft [28]. In Del Alamo et al [10] and Kuzmicz [15] BGN methods, the model parameters were calculated using the FermiDirac statistics. The simulator uses the Fermi-Dirac statistics in its calculations, however the bandgap model parameters are usually extracted using Boltzman statistics. Therefore, it becomes necessary to represent the bandgap narrowing terms as a sum of the apparant bandgap plus the correction. We have modified the model used in the original version of BiLow to account for the Fermi-Dirac statistics. After reviewing literature on BGN, we have found that theoretical [32], [46], [28], work as well as experimental [8], [13], [27], [48], [55] and [52], measurements show that the BGN in n- and p-type Silicon is the 
same. Therefore we have assumed in our work that the BGN is the same in n- and pregions. Also, all the three BGN models that we have used, have been extracted using the majority carrier concentration. Since we have assumed that the majority carrier concentration is equal to the ionized concentration of dopant atoms, the ionized impurity concentration as obtained from our incomplete ionization model and shown in Fig 6 has also been used to calculate the bandgap narrowing at temperature as low as $77 \mathrm{~K}$.

IV.3.1 Method I: ( del Alamo et al BGN model [8]): In lightly doped semiconductor, the product of the equilibrium hole and electron concentration ( $\mathrm{p}_{0}$ and $\mathrm{n}_{\mathbf{0}}$, respectively) is a constant that depends only on temperature :

$$
\mathrm{p}_{0} \mathrm{n}_{\mathrm{o}}=\mathrm{n}_{\mathrm{ie}}{ }^{2}(\mathrm{~T})
$$

where $n_{i e}$ is the intrinsic carrier concentration.

. The net physical effect is the increase of the $n_{o} p_{0}$ product. In other words, if a large number of electrons, $\mathrm{N}_{\mathrm{D}}$, are introduced , the resulting hole concentration in equilibrium is given by

$$
\mathrm{p}_{\mathrm{o}}=\frac{n_{i e}^{2}\left(T, N_{D}\right)}{N_{D}}
$$

where $\mathrm{n}_{\mathrm{ie}}>\mathrm{n}_{\mathrm{io}}$. nio is the intrinsic carrier concentration at equilibrium.

Therefore, $p_{0}$ in heavily doped silicon is higher than what could be from the eqn (77) for lightly doped semiconductor. Since $n_{i o}$ depends exponentially on the bandgap of the semiconductor, a popular way of mathematically treating the problem is to assume that a rigid narrowing of the bandgap is solely responsible for the increase of $\mathrm{p}_{0}$, such that

$$
\mathrm{p}_{0} \mathrm{~N}_{\mathrm{D}}=\frac{n_{i e}^{2}\left(T, N_{D}\right)}{N_{D}}=\mathrm{n}_{\mathrm{ie}}{ }^{2} \exp \Delta \mathrm{E}_{\mathrm{g}} \operatorname{app} \frac{\left(N_{D}, T\right)}{k T}
$$

where $\Delta \mathrm{E}_{\mathrm{g}}$ app represents the fictitious bandgap shrinkage that would account for the increase of $\mathrm{p}_{0}$ if no other heavy doping effects occured. In this equation, complete 
activation of dopant atoms is assumed. The following expression from Del Alamo et al [10] has been chosen to be added in the simulator.

$$
\Delta \mathrm{E}_{\mathrm{g}}=18.7 \times 10^{-3} \ln \left(\frac{N_{D}}{7 \times 10^{17}}\right) \mathrm{eV}
$$

Because at low doping concentration the BGN model was extracted using Boltzman statistics, we modified the apparant bandgap narrowing model to accommodate the Fermi-Dirac statistics at high doping concentration by using the Joyce-Dixon approximation [19]:

$$
\begin{aligned}
& \Delta \mathrm{E}_{\mathrm{g}}{ }^{a p p_{\mathrm{FD}}}=\Delta \mathrm{E}_{\mathrm{gB}}+\Delta \mathrm{E}_{\mathrm{gJD}} \\
& \Delta \mathrm{E}_{\mathrm{gJD}}=\mathrm{kT}\left[0.353553\left(\frac{N}{N_{x}}\right)-4.95009 \times 10^{-3}\left(\frac{N}{N_{x}}\right)^{2}\right]
\end{aligned}
$$

$\Delta \mathrm{E}_{\mathrm{gB}}$ is the $\mathrm{BGN}$ extracted from equation (80), $\mathrm{N}$ is the majority carrier concentration, and $N_{X}$ is the conduction band density of states for $n$-type material or the valence band density of states for p-type material.

IV.3.2 Method II: (Kuzmicz BGN model [27]): In this second method, we assumed that the density of states in the conduction band and the valence band to be parabolic and that the bandgap narrowing is identical in both $\mathrm{n}$ and $\mathrm{p}$-type Si. Therefore the bandgap narrowing in heavily doped $\mathrm{Si}$ includes the "rigid" bandgap shift, $\Delta \mathrm{E}_{\text {geff }}$ as well as an apparent bandgap widening due to the use of Fermi-Dirac statistics, $\Delta \mathrm{E}_{\mathrm{gN}}$. Thus, the total BGN [27] is given by :

$$
\Delta \mathrm{E}_{\mathrm{g}}=\Delta \mathrm{E}_{\mathrm{geff}}+\Delta \mathrm{E}_{\mathrm{gN}}
$$

Kuzmicz and Wosik have assumed that the bandgap narrowing versus doping dependence can be approximated by the function :

$$
\Delta \mathrm{E}_{\mathrm{geff}}=\mathrm{a} n_{m}^{\frac{1}{3}}
$$

where $\mathrm{n}_{\mathrm{m}}$ is the concentration of majority carriers $\mathrm{n}_{\mathrm{o}}=1.0 \times 10^{17} \mathrm{~cm}^{-3}$ and $\mathrm{a}$ is an empirical parameter. The estimate of $a$ is done from 


$$
\mathrm{a}=1.32 \times 10^{18} \frac{q^{2}}{\varepsilon_{0} \varepsilon_{s}} \mathrm{ev.cm}
$$

This gives for $\mathrm{Si}\left(\varepsilon_{\mathrm{S}}=11.7\right), \mathrm{a}=3.6 \times 10^{-8} \mathrm{eV} . \mathrm{cm}$.

The apparent bandgap narrowing $\Delta \mathrm{E}_{\mathrm{gN}}$ is given by

$$
\Delta \mathrm{E}_{\mathrm{gN}}=k T \ln \left[\frac{\exp (\eta)}{F_{1 / 2}(x)}\right]
$$

where $F_{1 / 2}-$ Fermi integral of order $1 / 2$ and $n$ is given by

$$
\mathrm{R}_{\mathrm{n}}=\frac{n_{\mathrm{m}}}{N_{s}}=\mathrm{F}_{1 / 2}(\eta)
$$

( $n_{m}$ is the majority carrier concentration, $N_{S}$ is the effective density of states in the majority carrier band).

$$
\mathrm{N}_{\mathrm{S}}=\mathrm{N}_{\mathrm{c}}=3.22 \times 10^{19}\left(\frac{T}{300}\right)^{\frac{1}{2}} \mathrm{~cm}^{-3}
$$

and $\mathrm{N}_{\mathrm{S}}=\mathrm{N}_{\mathrm{V}}=1.83 \times 10^{19}\left(\frac{T}{300}\right)^{\frac{1}{2}} \mathrm{~cm}^{-3}$

Density of states in the conduction band and in the valence band are assumed to be parabolic. Theoretically, a deviation from parabolicity can arise from several phenomena, including band tail formation and formation of impurity bands, which can merge into majority carrier band. However numerous experiments [8], [18] prove that the assumption of band parabolicity is sufficiently accurate in most cases. Thus $\triangle \mathrm{EgN}$ can be easily determined using a simple and accurate approximation of the Fermi-Dirac integral.

Equations (86) and (87) can be combined using the approximation of Nilsson [60] for the Fermi integral

$$
\Delta E_{g N}=k T R_{n}\left[64+0.05524 R_{n}\left(64+R_{n}\right)\right]^{-1 / 4}
$$


IV.3.3. Method III (original BGN model in BiLow [5]): The following expression has been used in the original BiLow [5] for the purpose of modeling BGN

$$
\Delta \mathrm{E}_{\mathrm{gB}}=A \ln \left(\frac{N}{N r e f}\right) \quad 18 \times 10^{-3}<\mathrm{A}<19 \times 10^{-3}
$$

or n-type material

$$
\mathrm{N}_{\text {ref }}=\mathrm{N}_{\text {ref }}(300 \mathrm{~K}) \frac{T}{300 K}\left\{1+2 \exp \left(\frac{E_{F n}-E_{D}}{k T}\right)\right\}
$$

For p-type material

$$
\mathrm{N}_{\text {ref }}=\mathrm{N}_{\text {ref }}(300 \mathrm{~K}) \frac{T}{300 K}\left\{1+4 \exp \left(\frac{E_{A}-E_{F_{p}}}{k T}\right)\right\}
$$

where $A=18 \times 10^{-3}$ and $\operatorname{Nref}(300)=7 \times 10^{17} \mathrm{~cm}^{-3}$ were used Using the $\Delta \mathrm{E}_{\mathrm{gJD}}$ from eqn (82), we get

$$
\begin{aligned}
\Delta \mathrm{E}_{\mathrm{g}} \mathrm{app}_{\mathrm{FD}}= & \Delta \mathrm{E}_{\mathrm{gB}}+\Delta \mathrm{E}_{\mathrm{gJD}} \\
\Delta \mathrm{E}_{\mathrm{g}} \mathrm{app}_{\mathrm{FD}}= & A \ln \left(\frac{N}{N_{\text {ref }}}\right)+\mathrm{kT}\left[0.353553\left(\frac{N}{N_{x}}\right)\right. \\
& \left.-4.95009 \times 10^{-3}\left(\frac{N}{N_{x}}\right)^{2}\right]
\end{aligned}
$$

\section{IV.4 Results.}

We have already shown in Fig 9 the collection of reported experimental data on $\Delta$ Egapp. We insist in denoting this parameter as " apparant bandgap narrowing " because of the way it is modeled through eqn.(79). The relationship between $\Delta \mathrm{Eg}^{\mathrm{app}}$ and any real shrinkage of the fundamental gap is far from clear. The dramatic discrepancies among theory [2], [20], [32] and [46], electrical [10] and luminescence [55] measurements indicate that a complete understanding of the band structure of heavily doped silicon is lacking. We have calculated the bandgap narrowing using Methods I, II 
and III. Method I and Method II have been included in the simulator in the form of two separate subroutines. Temperature correction has been added to the existing Method III. However, the simulator program has the capacity to chose any model at a time. In Fig 15 we compare the room temperature values of bandgap narrowing $\Delta \mathrm{Eg}$ as calculated using Method I, Method II and the original Method III from BiLow. The bandgap values calculated using Method II are larger than those from Method I. This discrepancy is

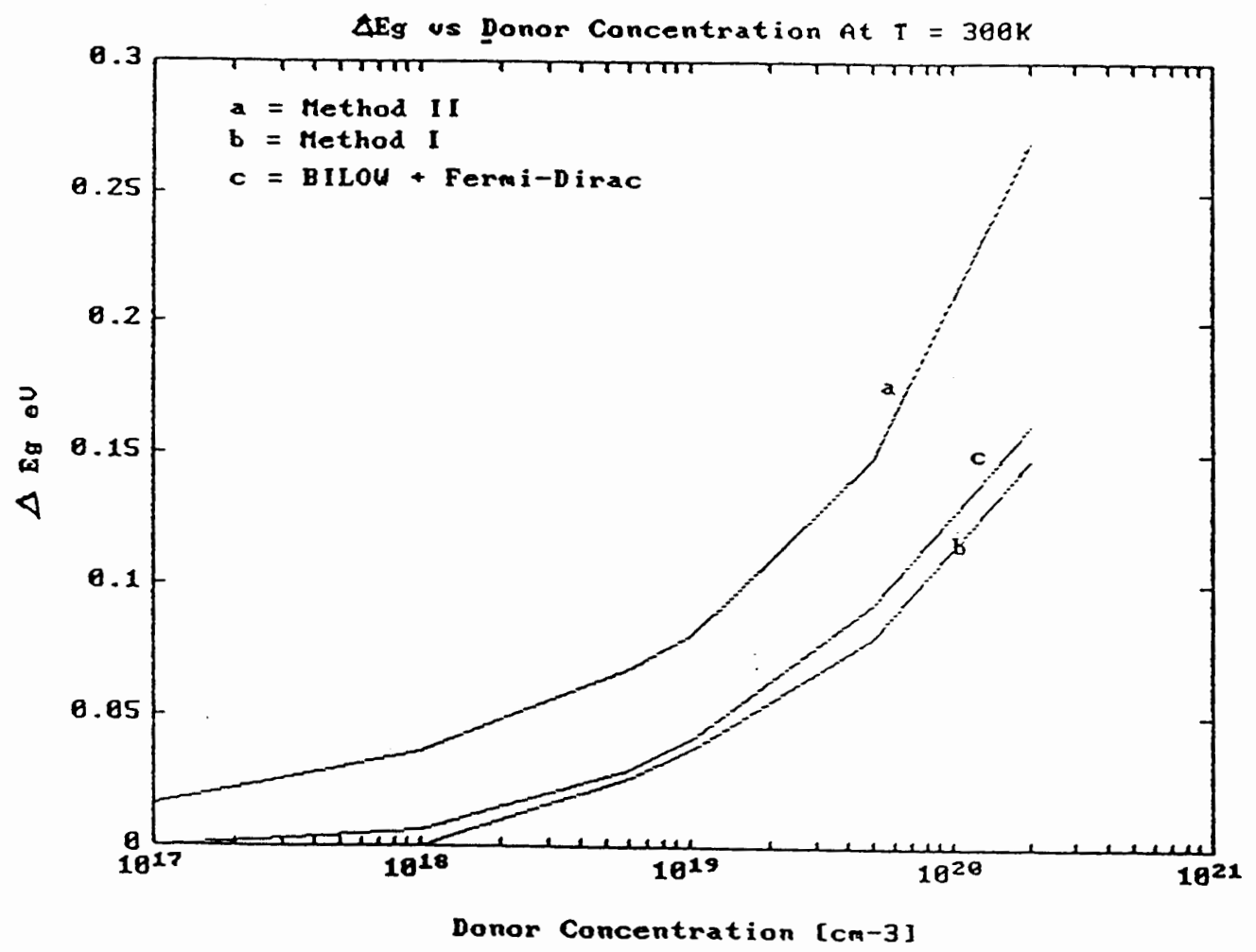

Figure 15. Bandgap narrowing at $\mathrm{T}=300 \mathrm{~K}$ as a function of temperature

mainly due to the different asumptions made about the carrier mobility. In Method II, it is assumed that the mobility of minority electrons is equal to the mobility of majority electrons. Whereas, in Method I, it is assumed that the hole mobility in n-type silicon, as minority carrier, is about two times larger than as majority carrier in p-type silicon. It has 
also been assumed in Methods I, II and III that all the dopant atoms are completely ionized and that the bandgap narrowing is the same in n-type and p-type silicon. Using the ionized fraction as a function of temperature and concentration, as obtained from our Mott transition model described in Chapter III, section III.2, we can now investigate the bandgap narrowing as a function of temperature.

In Fig 16, we have plotted the bandgap narrowing extracted from the models

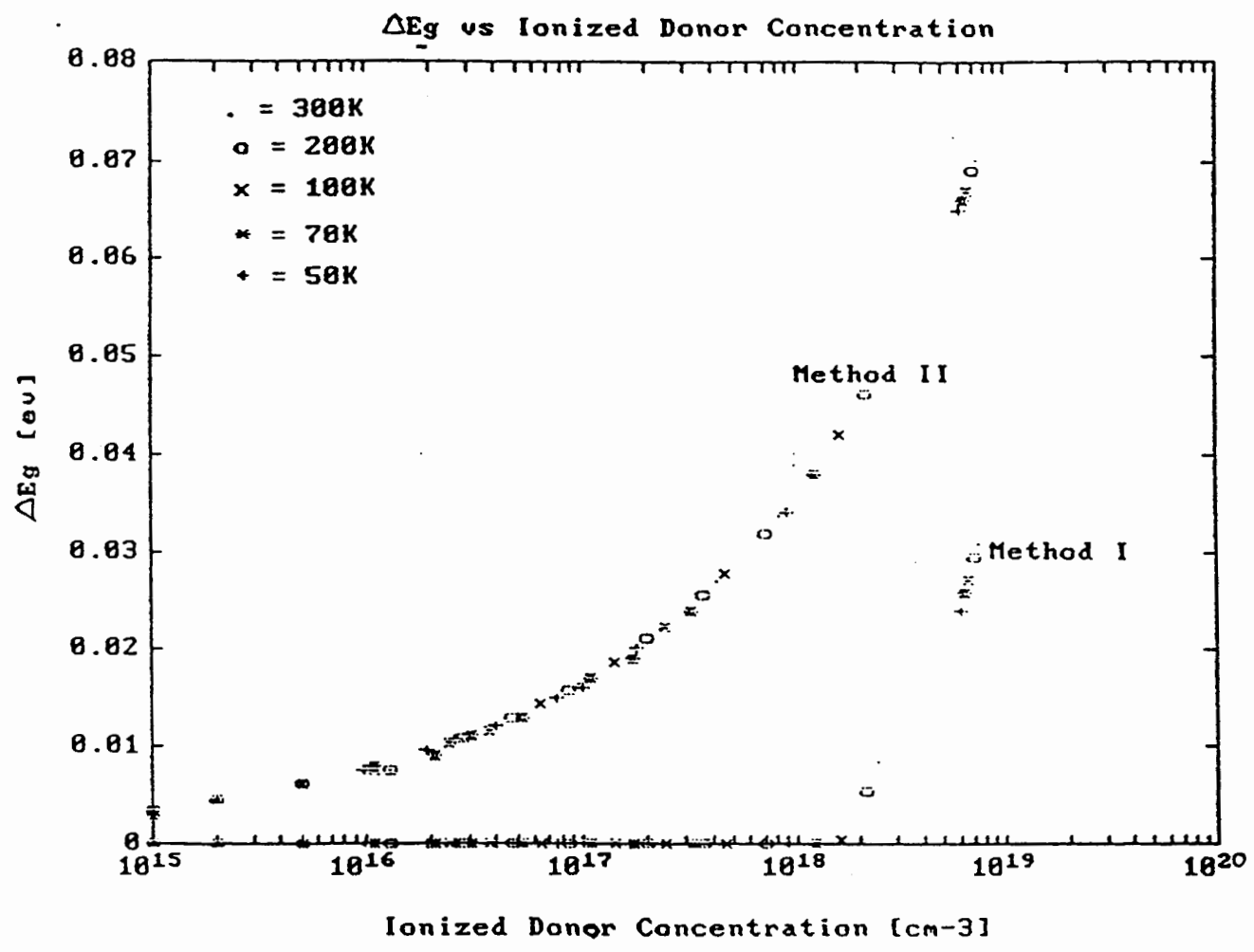

Figure 16. Bandgap narrowing as a function of ionized donor concentration and temperature

described above as a function of temperature and ionized impurity concentration. Within an accuracy of plus-minus $10 \mathrm{meV}$, the same bandgap narrowing is found at $50 \mathrm{~K}$ and at $300 \mathrm{~K}$. This is in good agreement with the optical PL results of J.Wagner et al. [55] and the experimental measurements of Klassen et al.[23]. The present values of the bandgap 
narrowing have been derived assuming parabolic bands and do not include additional narrowing effects, which may arise from band tailing in the majority-carrier bands. Fig 17 shows the bandgap narrowing as a function of donor concentration which was obtained from the impurity ratio of the ionization model. In the literature survey on BGN,

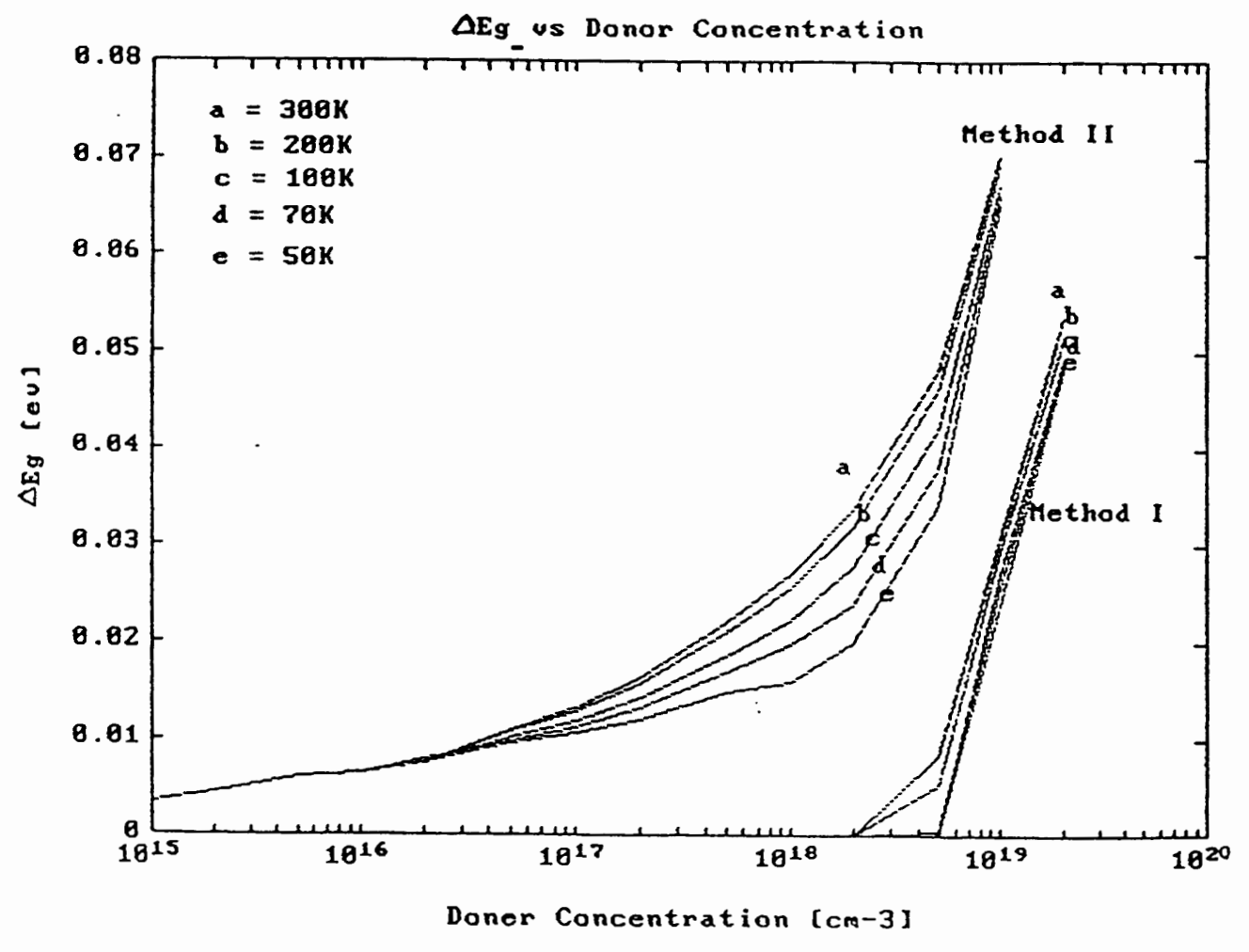

Figure 17. Bandgap narrowing as a function of donor concentration and temperature

we found that Jain and Roulston [20] have predicted a temperature dependency of BGN and have added a temperature correction to their BGN data. Comparing the BGN values from Fig17 with the theory of Jain et al [20] in Fig 14, we see that at low concentration the difference between the $\mathrm{BGN}$ values is as large as $30 \mathrm{meV}$, but at high concentrations of about $10^{19} \mathrm{~cm}^{-3}$ the difference is only about $5 \mathrm{meV}$. This may be due to the fact that all 
in Fig 14, we see that at low concentration the difference between the BGN values is as large as $30 \mathrm{meV}$, but at high concentrations of about $10^{19} \mathrm{~cm}^{-3}$ the difference is only about $5 \mathrm{meV}$. This may be due to the fact that all interactions have not been properly taken into account in calculating the BGN in the rigid parabolic band approximation by Jain et al. [20]. The temperature dependence is dominant from $1 \times 10^{17}$ to $5 \times 10^{18} \mathrm{~cm}^{-3}$ for Method II and from $1 \times 10^{18}$ to $2 \times 10^{19} \mathrm{~cm}^{-3}$ for the Method I. In these ranges the impurity atoms are not ionized. The model described in Method I shows an abrupt increase in the bandgap at $4 \times 10^{18} \mathrm{~cm}^{-3}$. This is not visible in Method II where the increase is gradual. Further, Method II is well described for low and moderate doping concentrations. One major difference between the two models is that Method I describes the BGN model at room temperature and extends it to lower temperatures. It assumes the density of states value at room temperature. In Method II, the effective density of states are described by the eqns (92), (93) and are temperature dependent. Simulations were done using the three models described in Section IV.3. The results of the simulations using the ionization model and the bandgap narrowing model are presented in the final Chapter V. 


\section{CHAPTER V}

\section{LOW TEMPERATURE BIPOLAR TRANSISTOR SIMULATION}

\section{V.1. Simulator Program Description}

The simulator program, is a modified version of BiLow [5] converted from original FORTRAN-version to the C-version. It is a modular program that separates the algorithms that need to be experimented with, from the main body that does the iterative convergence needed to arrive at the solutions. It accepts user input interactively for parameters that determine the conditions of the program execution while using file input/output for most of the subsequent transfers. The input to the simulator is the simulation temperature, the doping profile and the initial guess file. The advantage of using the dynamic memory allocation facility makes the program consume less memory than the original. The simulator implementation is based on a variety of mathematical techniques [45]. Since the FORTRAN version of BiLow [5] used math funtions from the FORTRAN library, new subroutines had to be written in the C-version to substitute for these standard FORTRAN options. Two modifications were made to the new program on

the basis of the models which have been described in the earlier Chapters. The "make" file has been written to choose the model inclusion. Developed on a Sun Sparcstation, this program does not use any processor specific features and could be rebuilt on any platform where a $\mathrm{C}$ compiler is supported. The main BiLow program consists of about 3000 lines of code in addition to twenty subroutines. 


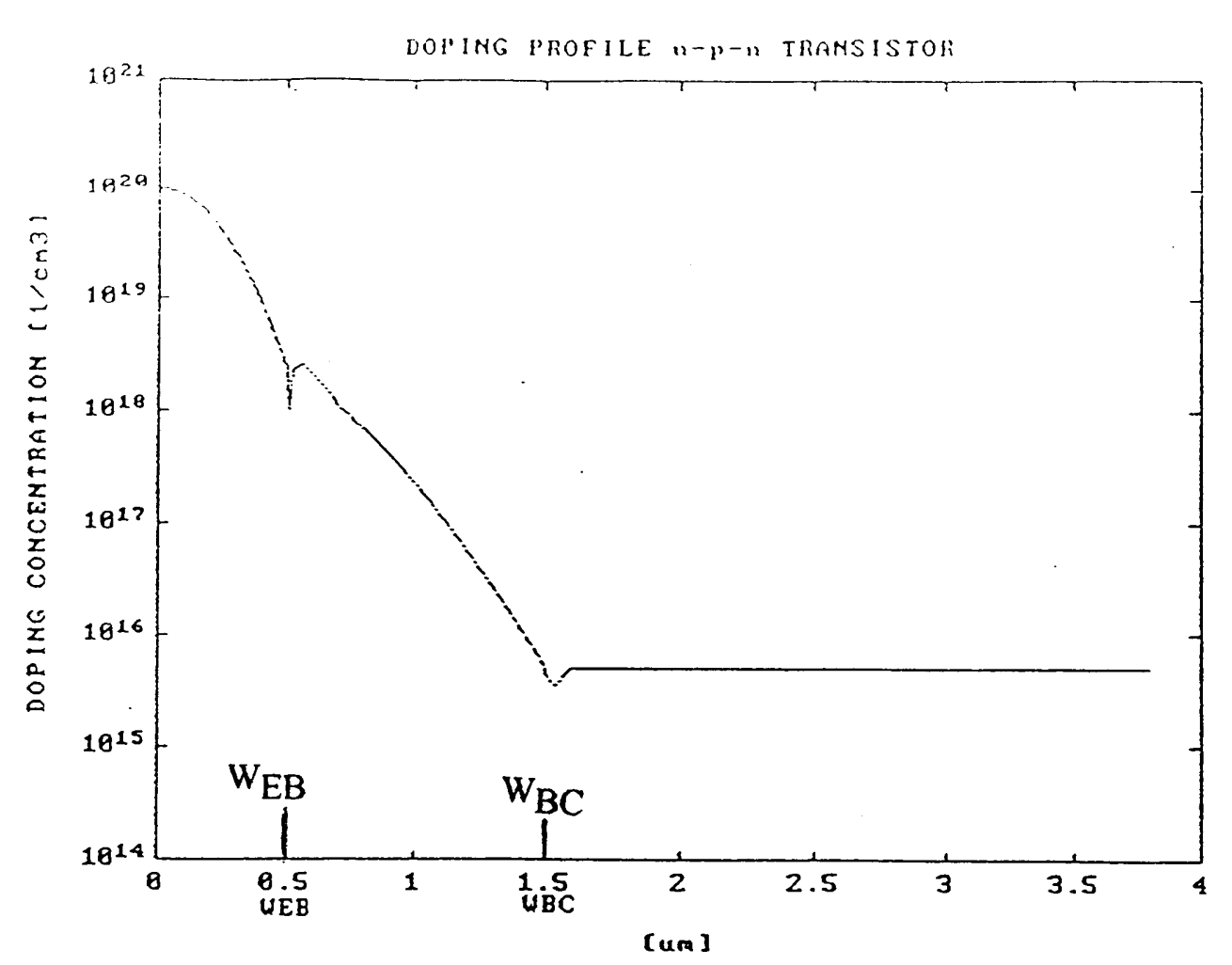

Figure 18. Doping profile of an n-p-n transistor T1

\section{V.2. Simulator Performance}

Two double diffused n-p-n bipolar transistor doping profiles were used in our simulations using the new BiLow. The profiles were generated using a double gaussian function given below

$$
N(x)=N_{e} \exp -\left(\frac{x}{x_{e}}\right)^{2}-N_{b} \exp -\left(\frac{x}{x_{b}}\right)^{2}+N_{e p i}
$$

The doping profile of the transistor $\mathrm{T} 1$ neglects the contribution of the $\mathrm{N}_{\mathrm{epi}}$ and is shown in Fig 18. The total number of grid points used in the simulator were 187 grid points and these can be further reduced or increased depending on the doping profile. The number of iterations needed for convergence depends on the doping profile, number of mesh points and their distribuiton, bias voltages, temperature, parameter models, and the algorithm used. Bias condition used for higher temperature had to be used as initial 
conditions to obtain a solution for the temperature lower than $80 \mathrm{~K}$. Once convergence was obtained for a given tempeature less than $80 \mathrm{~K}$, the solution was then used as the initial condition for the higher bias voltages as well as for lower temperatures. The flowchart for the operation of the simulator is given in Fig 3 of Chapter II. Table III presents the number of iterations that are needed to achieve convergence for different temperatures and different bias conditions. For large voltage steps, the simulator fails to converge. For voltage biases smaller than the maximum voltage showed in Table III, the number of iterations is the same as shown in Table III for a variety voltage steps. The results in Table III are the same for transistors $\mathrm{T} 1$ and $\mathrm{T} 2$.

TABLE III

CONVERGENCE STATISTICS

\begin{tabular}{lllllll}
\hline Number of iterations & $\begin{array}{l}300 k \\
\text { VBE [v] }\end{array}$ & $\begin{array}{l}150 k \\
\text { VBE [v] }\end{array}$ & $\begin{array}{l}122 k \\
\text { VBE [v] }\end{array}$ & $\begin{array}{c}100 k \\
\text { VBE [v] }\end{array}$ & $\begin{array}{c}77 k \\
\text { VBE [v] }\end{array}$ \\
\hline-300 & $<0.84$ & $<0.9$ & $<0.9$ & $<1.00$ & $<1.00$
\end{tabular}

\section{V.3. Simulator Results}

Sample results of bipolar transistor simulation in the active region in which the emitter-base junction is forward biased and the base-collector junction is reverse biased. are presented below. The simulations were performed by including the incomplete ionization model described in Chapter III and the bandgap narrowing model of Method II given in Chapter IV. Simulations were also performed using the other two BGN models, Method I and Method III presented in Chapter IV. The charge characteristics versus current densities for 300-100K temperature 


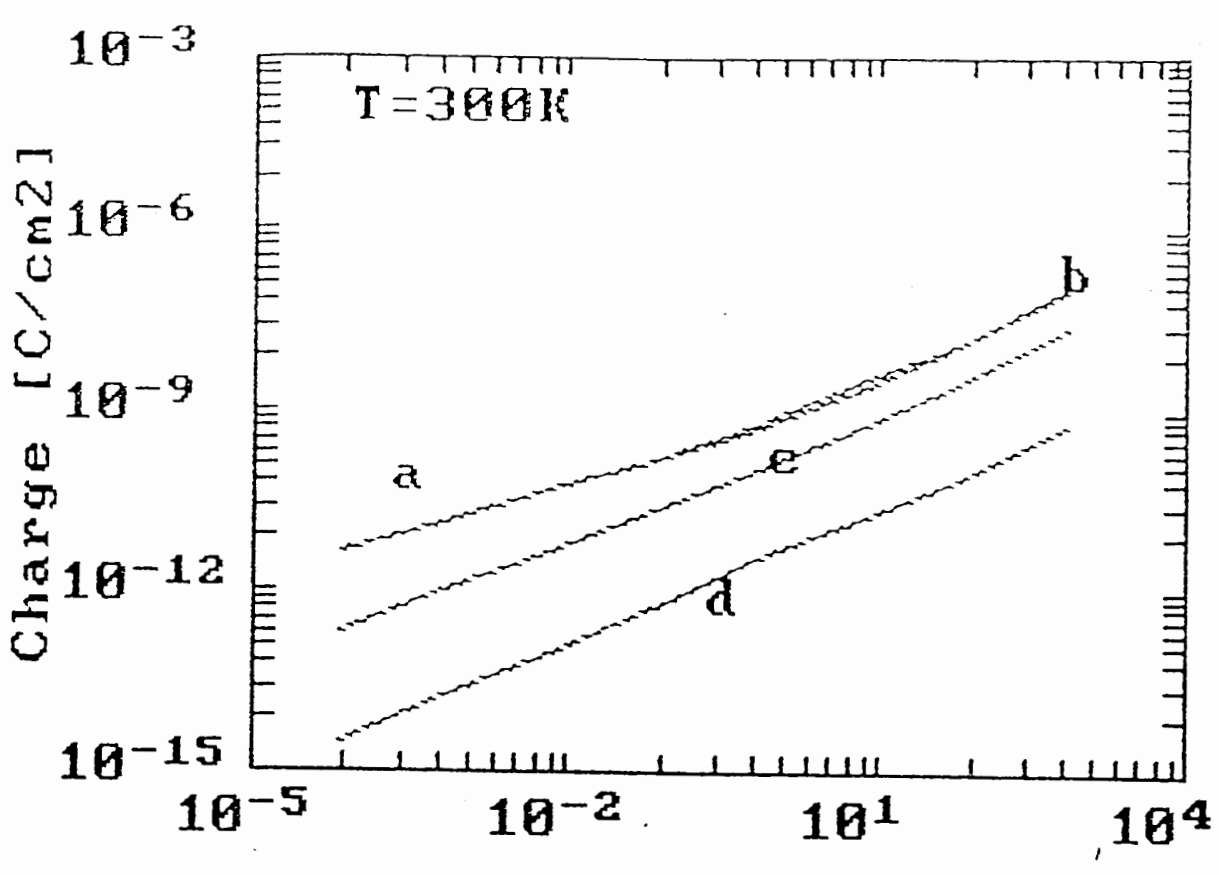

Current Density $[\mathrm{A} / \mathrm{cm} 2]$

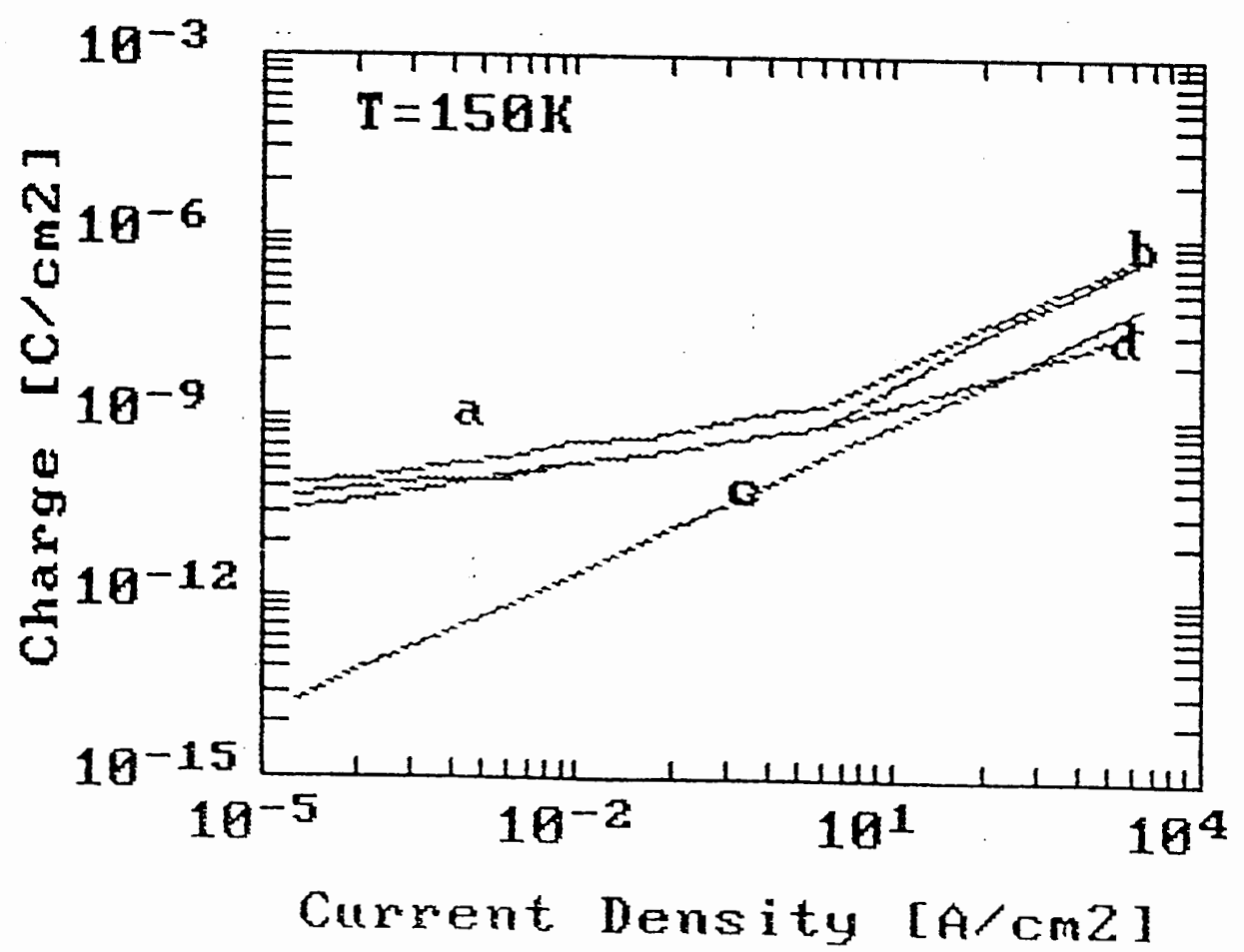

Figure 19. Charge Characteristics at (a) $\mathrm{T}=300 \mathrm{~K}$ and (b) $\mathrm{T}=150 \mathrm{~K}$. $\mathrm{a}=$ total charge, $b=$ charge of electrons in base, $c=$ charge of holes in emitter, $d=$ charge of donors trapped in base. 

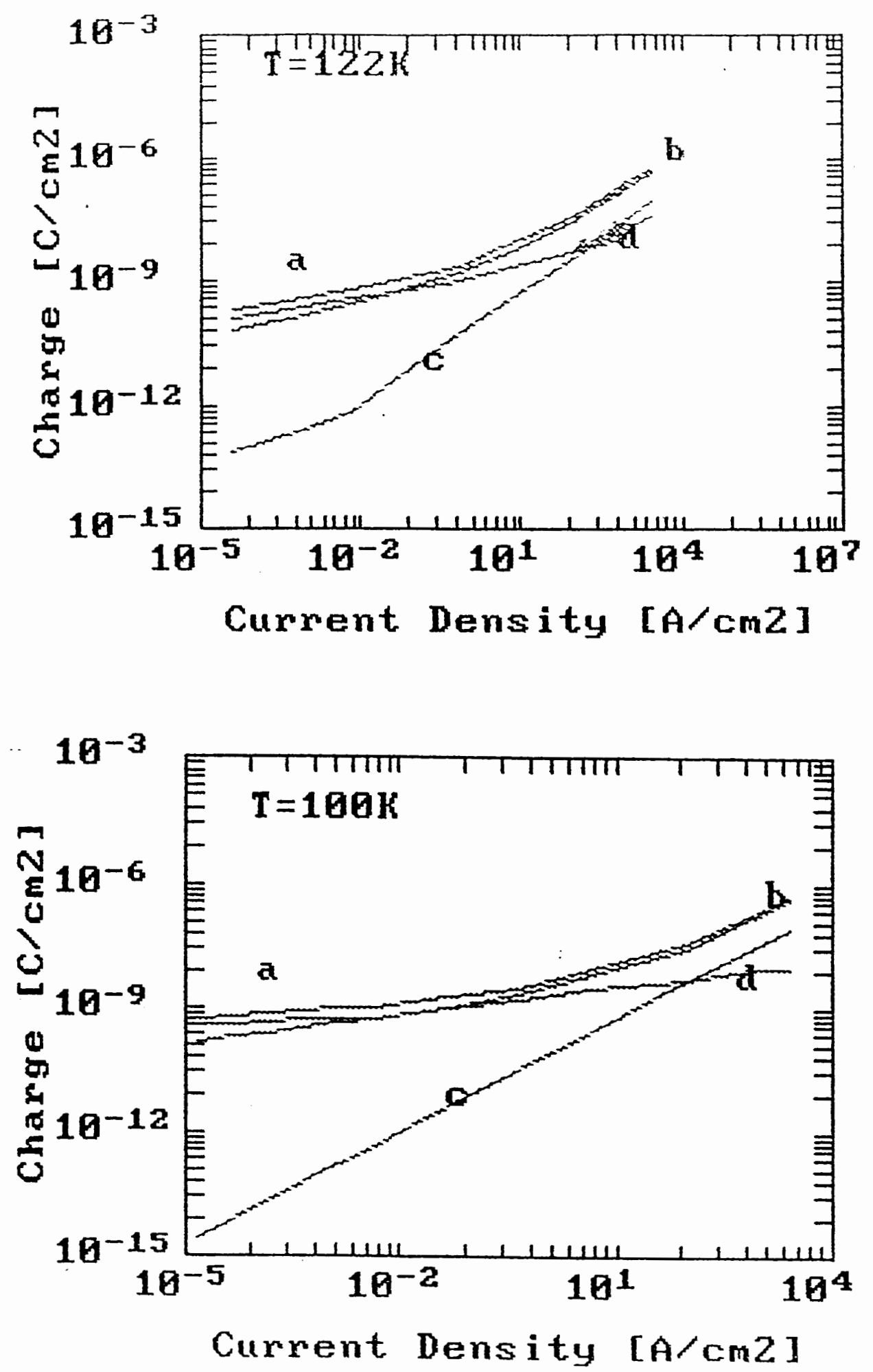

Figure 20. Charge Characteristics at (a) $\mathrm{T}=122 \mathrm{~K}$ and (b) $\mathrm{T}=100 \mathrm{~K}$. a = total charge, $b=$ charge of electrons in base, $c=$ charge of holes in emitter, $d=$ charge of donors trapped in base. 
range were generated. Fig 19a, Fig 19b, Fig 20a, Fig $20 \mathrm{~b}$ show the data for $\mathrm{T}=300 \mathrm{~K}, \mathrm{~T}$ $=150 \mathrm{k}, \mathrm{T}=122 \mathrm{~K}$ and at $\mathrm{T}=100 \mathrm{~K}$ for the transistor $\mathrm{T} 1$ respectively. Under the "active region" bias conditions holes will be injected into the n-type emitter from the base; electrons will be injected from the n-type emitter into the p-type base. Fig 21 shows the minority carrier distributions in an $n-p-n$ transistor. The $x$-axis represents the distance along the length of the device. The origin is chosen where the active base begins. The end of the active base, at the base edge of the collector depletion region, is at $\mathrm{x}=\mathrm{w}$. The other edges of the two depletion regions are at $x=-x_{E}$ for the emitter and $x=x_{C}$ for the collector. The distribution of the minority carriers across the device is shown assuming low level injection condition. Since the base-collector region is reversed biased, the minority carrier concentration on each side of this depletion layer is zero. The collector acts as a "sink" for the electrons diffusing across the base. The electric field within the base-collector space charge region will carry the electrons through to the $\mathrm{N}$ type collector giving rise to a collector current $I_{c}$ which is as large as the forward-bias current of the emitter-base junction $I_{E}$.

To explain the charge characteristics as a function of current density we have drawn in Fig 22(a)-(g), the minority carrier distribution in the emitter and the base of an $n-p-n$ transitor with homogeneous doping profile for high and low injection levels. The plots are for increasing base-emitter voltage and a constant reverse bias voltage on the basecollector junction. The electron and hole concentration in the base and the emitter are $n(x)$ and $p(x)$ respectively. At $T=300 K$, and at small bias voltage on the base-emitter junction, the total charge consists mainly of the electron injected into the base whereas the hole current is negligible and this can be seen in Fig 22(b). However at high current density, space charge region in the base increases and moves towards the collector, increasing the base width. The current in the base region increases and the slope of the concentration is steeper than that at low level injection condition. 
When the collector current is low the electrons crossing the C-B junction are less in number leading to a low level injection condition. However, as the current increases, the density of electrons transported across the C-B junction increases and at some point becomes comparable to the doping on the collector side of the space charge region. this condition is known as high level injection condition.

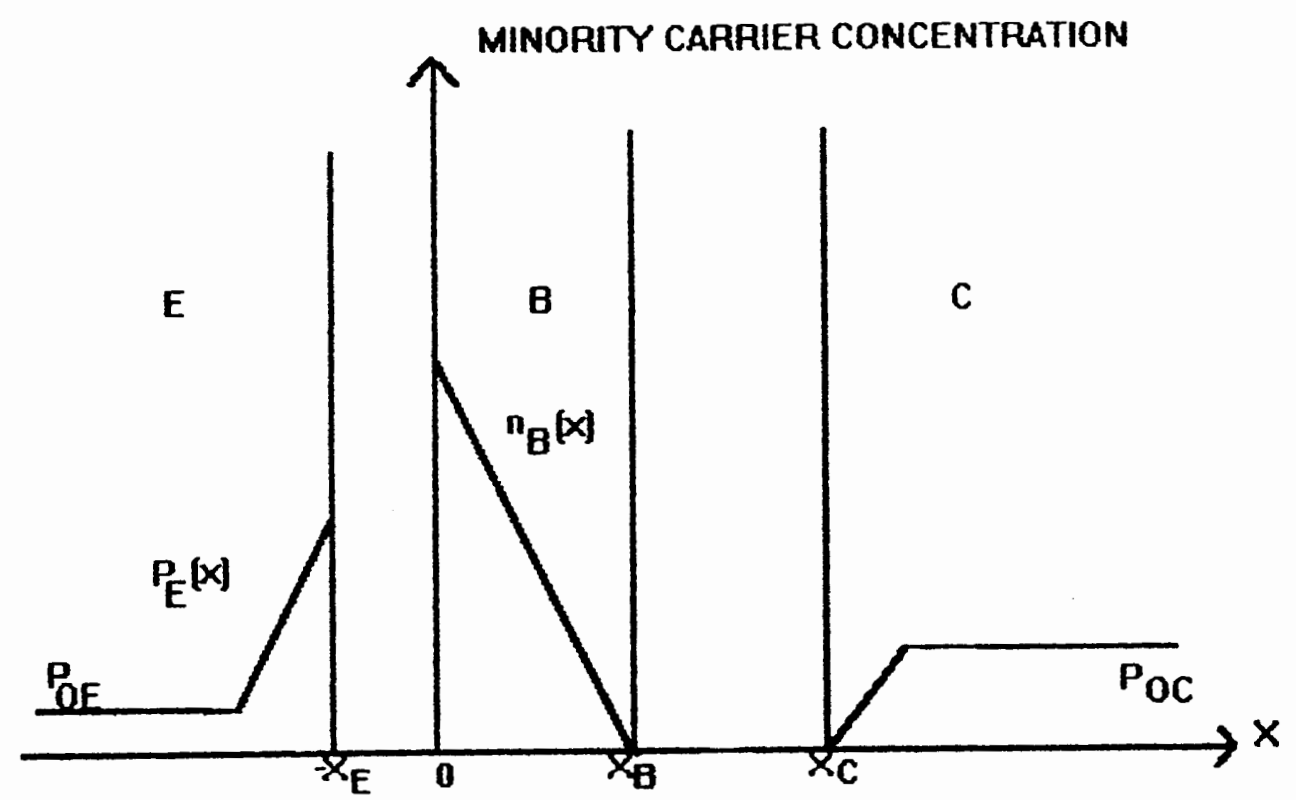

Figure 21. Minority carrier distribution in n-p-n transistor

This is shown in Fig 22(c) and the total charge increases but is still mainly composed of the charge of the electrons injected into the base. In Fig $20 \mathrm{a}$, at $\mathrm{T}=122 \mathrm{~K}$, the total charge at low level injection condition is larger than the total charge at $\mathrm{T}=300 \mathrm{~K}$ and at the same collector current. This is because at $\mathrm{T}=122 \mathrm{~K}$ and for small voltages, the total charge is the sum of the free electrons and the electrons trapped on the donor sites in the base. In Fig 22(d) the slope of the minority concentration in the base remains the same as in Fig 22(b), eventhough the trapped electron concentration increases. There is also a small increase in the hole concentration due to back injection of holes into the emitter. As the 
current density increases, the total charge is now the sum of the charges of the trapped electrons plus the free electrons in the base as well as the charge of the holes back injected into the emitter. Fig 22(e) shows the minority carrier distribution at higher collector current density. The charge distibution at $\mathrm{T}=77 \mathrm{~K}$ can be explained using Fig 22(f) and Fig 22(g). In Fig 22(f), the condition for low current density shows that the total charge is now mainly due to the electrons trapped on the donor sites in the base and the holes in the emitter, whereas the total charge at high injection condition in Fig 22(g) is mainly due to the holes back injected into the emitter. Comparing the charge characteristics plots of Fig 19(a)-(b) and Fig 20(a)-(b) with those obtained using the original BiLow [6] shown in Fig 24, Fig 25, and Fig 26, we see that the same trend as seen in Fig 19 through Fig 20, continues.

\section{Explaination of charge characteristics}

a)

\begin{tabular}{|l|l|l|}
\hline$E$ & $B$ & $C$ \\
\hline
\end{tabular}

$n-p-n$ Transistor

b)

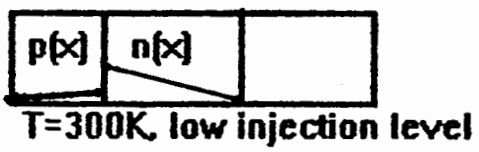

d)

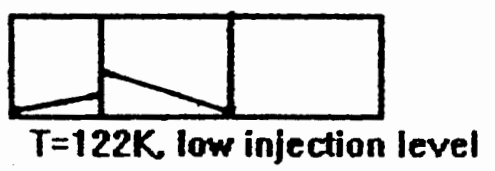

i)

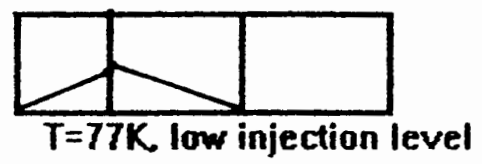

c)

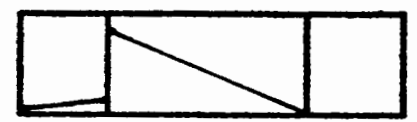

$T=300 \mathrm{~K}$, high inlection Ievel

e)

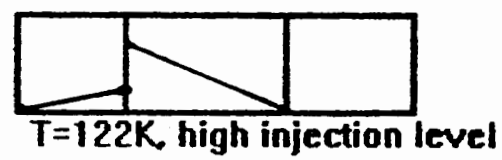

g)

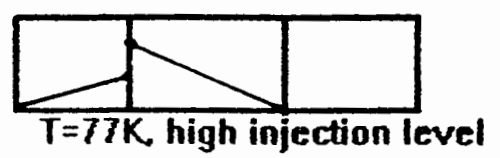

Figure 22 Minority carrier distribution to explain the charge characteristics 


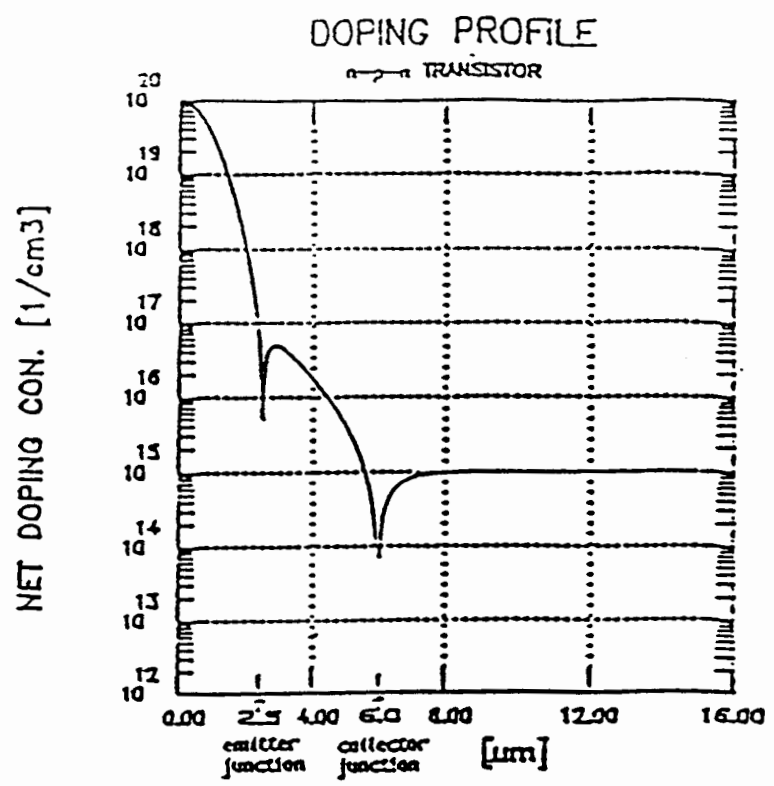

Figure 23. $n-p-n$ doping profile [6]

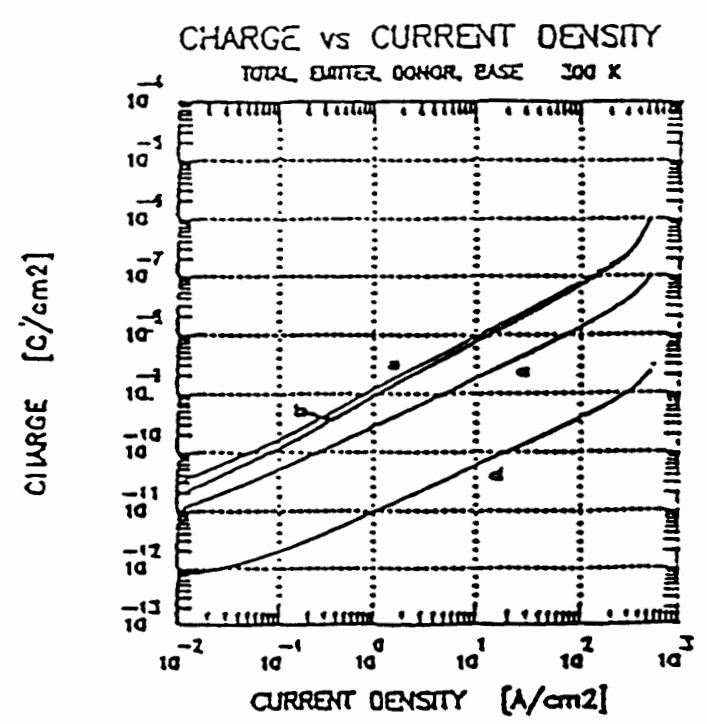

Figure 24. Charge characteristics at $\mathrm{T}=300 \mathrm{~K}[6]$

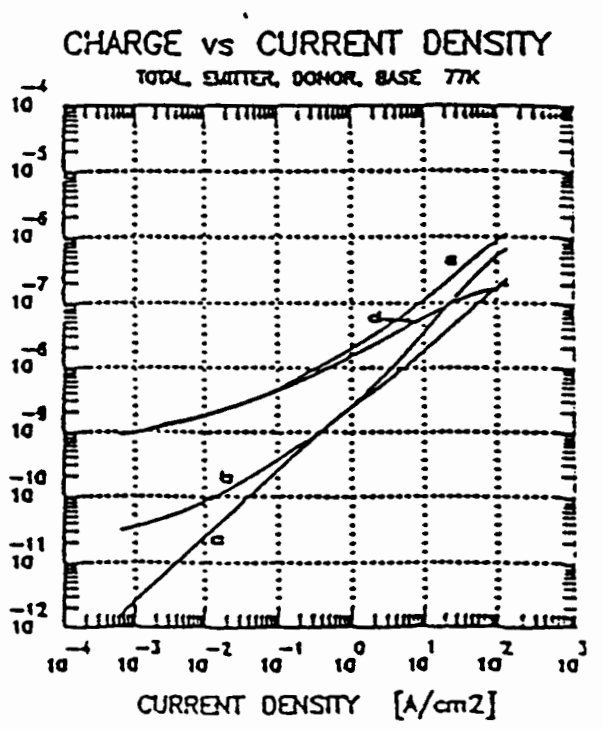

Figure 25. Charge characteristics at $\mathrm{T}=122 \mathrm{~K}[6]$

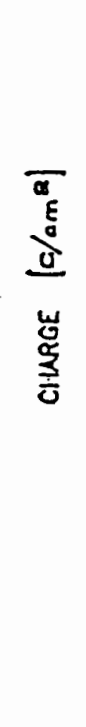

Figure 26. Charge characteristic at $\mathrm{T}=77 \mathrm{~K}[6]$ 
At $\mathrm{T}=300 \mathrm{~K}$, the charge of the free electrons in the base of $\mathrm{T} 1 \mathrm{in}$ Fig 19(a) is larger than the charge in Fig 24. At $T=122 \mathrm{~K}$ in Fig 20 (a), the total charge in the transistor $\mathrm{Tl}$ is larger the total charge of Fig 25. Comparing the doping profiles of transistor T1 from Fig 18 and the profile in Fig 23, we see that the total charge in the transistor which depends on the doping concentration in the emitter and the base is larger in case of transistor $\mathrm{T} 1$. This is due to the smaller size as well as higher doping in the base and the emitter of T1 as compared to the doping profile of the transistor shown in Fig 23. Electron distribution for high-level injection conditions are ploted in Fig 27. As the collector current density in an n-p-n transistor increases, the density of electrons being transported across the C-B space-charge region also increases. When this density becomes comparable to the doping on the collector side of the space-charge region, the total charge in this region becomes significantly reduced, leading to a lower electric-field gradient in the $C-B$ junction. With a lower maximum electric field strength, the space-charge region edge in the base moves towards the collector, effectively increasing the base width. This phenomenon is known as base push-out effect apparant at $\mathrm{T}=300 \mathrm{~K}$ and at high injection level. In addition, larger base width also reduces the $\mathrm{f}_{\mathrm{T}}$ and the transistor speed. At low temperature and at low current densities, the decrease of $f_{T}$ can be related to the increase in the charge of the electrons trapped at the donor sites. At higher current densities and at low temperature, the decrease of $\mathrm{f}_{\mathrm{T}}$ is mainly due to the increase in the charge of holes back injected into the emitter. Fig 28 gives the electron concentration for high level injection conditions as reported by Chrzanowska-Jeske and Jaeger [6]. On comparing Fig 27 and Fig 28, we see that the base push out effect which is prominant at $\mathrm{T}=300 \mathrm{~K}$ in both cases has vanished at lower temperatures. This is because as the temperature goes down, the number of free electrons in the base get trapped on the donor sites, and the density of electrons being transported across the C-B space-charge region decreases and there is no more increase in the base width. Therefore, at room temperature and at high current densities, the base 
push out effect contributes to the decrease in $f_{T}$ because of the increase in the base width which affects the base transient time. As the temperature decreases the base push out effect vanishes and the decrease in $\mathrm{f}_{\mathrm{T}}$ at low injection levels is now mainly due to the charge of the trapped electrons in the base. At high injection levels and at low temperatures the decrease in $\mathrm{f}_{\mathrm{T}}$ is due to the increase in the charge of the holes bcak injected into the emitter.

Another important characteristic of the bipolar transistor is its ability to provide current gain at high frequency. The unity gain frequency is the frequency of the transistor at which the current gain decreases to unity and it can be related to the physical structure of the transistor through the transient time $\tau_{\mathrm{ec}}$ of the transistor [6]

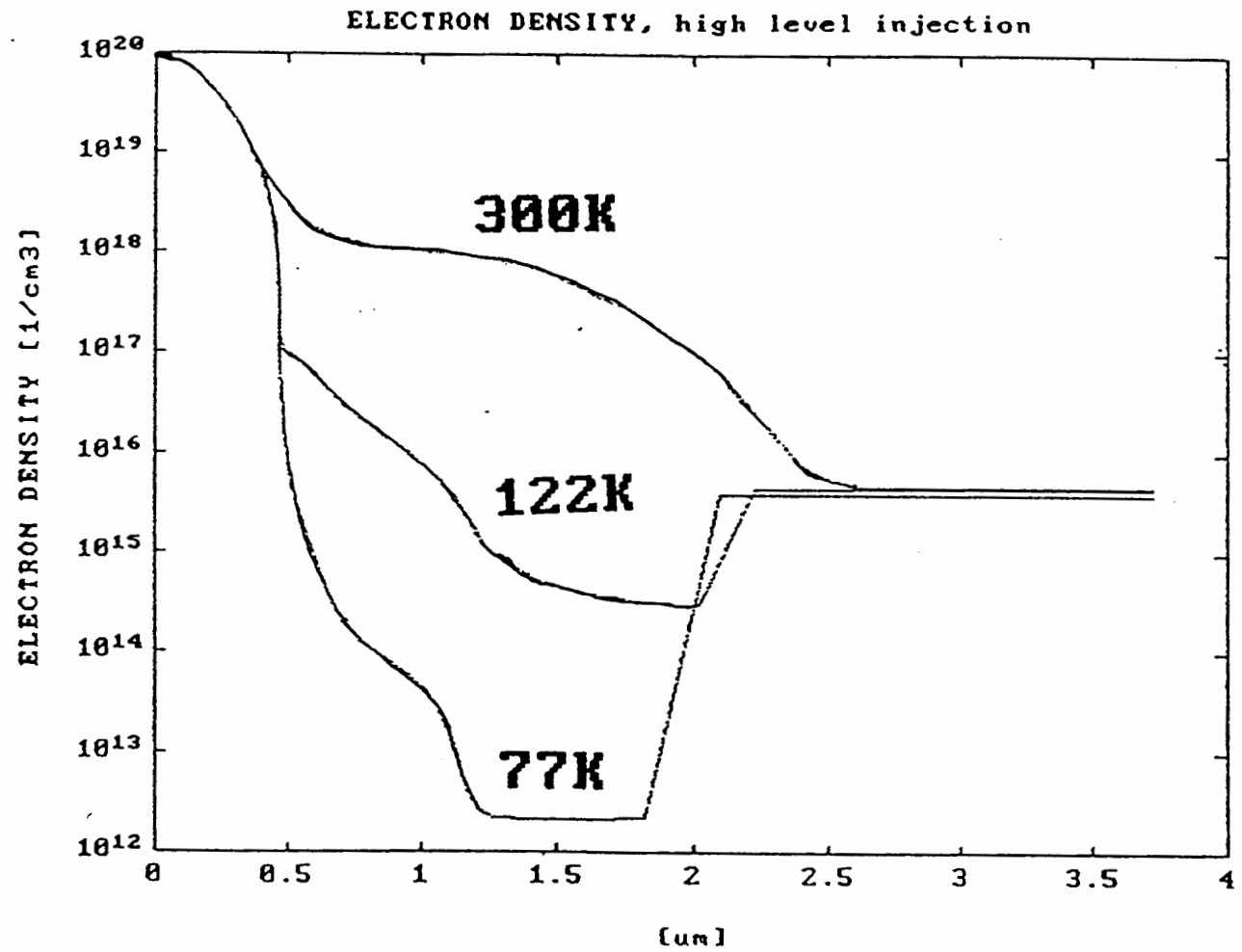

Figure 27. Electron concentration at different temperatures for high level injection: $\mathrm{J}(300 \mathrm{~K})=480 \mathrm{~A} / \mathrm{cm}^{2}, \mathrm{~J}(122 \mathrm{~K})=800 \mathrm{~A} / \mathrm{cm}^{2}$, $\mathrm{J}(100 \mathrm{~K})=65 \mathrm{~A} / \mathrm{cm}^{2}$ 


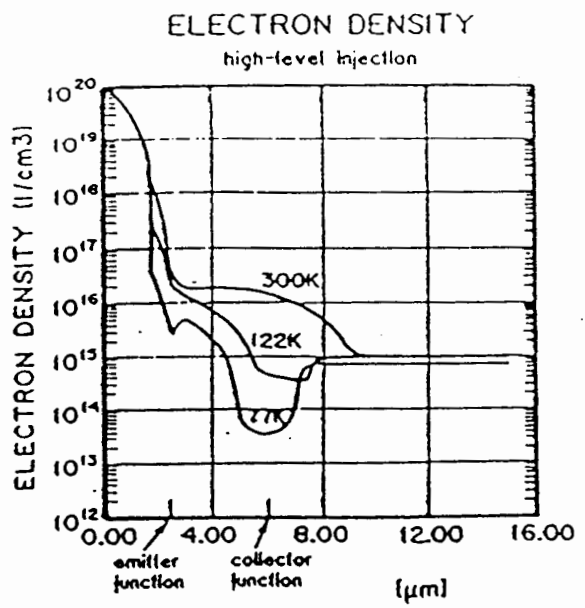

Figure 28. Electron concentration at different temperatures for high level injection: $\mathrm{J}(300 \mathrm{~K})=550 \mathrm{~A} / \mathrm{cm}^{2}, \mathrm{~J}(122 \mathrm{~K})=840 \mathrm{~A} / \mathrm{cm}^{2}$, $\mathrm{J}(77 \mathrm{~K})=65 \mathrm{~A} / \mathrm{cm}^{2}[6]$

$\mathrm{f}_{\mathrm{T}}=1 / 2 \pi \tau_{\mathrm{ec}}$

The delay time $\tau_{\mathrm{ec}}$ is the sum of the emitter storage time and the base transit time.

The emitter storage time is given by [6]

$$
\tau_{e}=\frac{Q_{p}}{I_{E}} \quad \mathrm{Q}_{\mathrm{p}} \text {-holes in the emitter }
$$

The base transient time is given by [6]

$$
\tau_{B}=\frac{\left(Q_{n f}+Q_{n t}\right)}{I_{c}}
$$

$\mathrm{Q}_{\mathrm{nf}}$ is the total charge of free electrons in the base and $\mathrm{Q}_{\mathrm{nt}}$ is the charge of the electrons trapped on the donor sites. For minimum value of $\tau$ to be obtained, several features of the bipolar transistor structure have to be optimized. The base width should be as narrow as possible, buried layers and deep-collector contacts are used to minimize $R_{c}$. The simulated temperature dependence of the peak $\mathrm{f}_{\mathrm{T}}$ is shown in Fig.29. From our simulated results, the peak $f_{T}$ is a function of temperature with a maximum around $T=150 \mathrm{~K}$. From Fig 29 , it looks like the temperature dependence of the peak $f_{T}$ occurs at temperature 


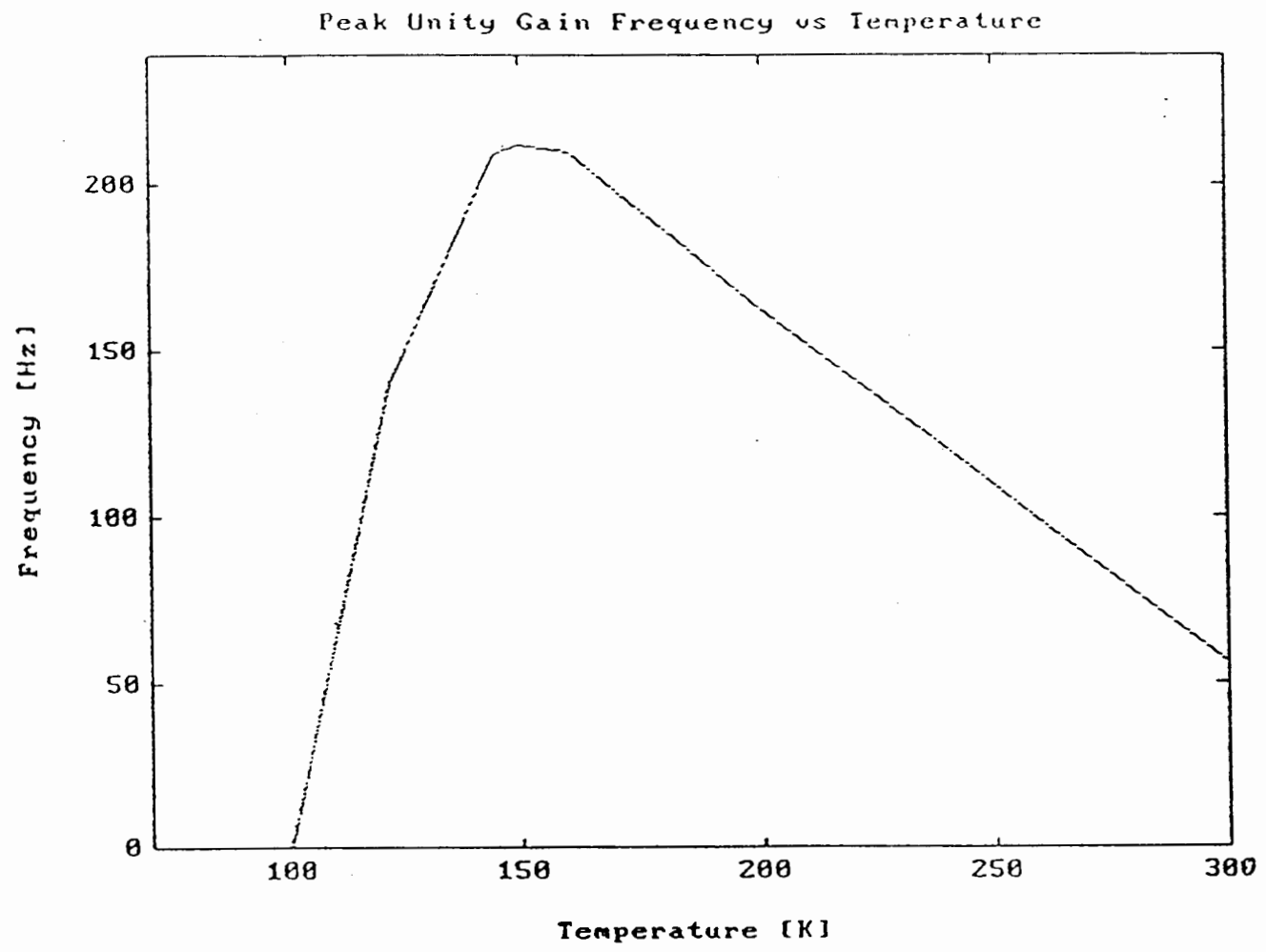

Figure 29. Temperature dependence of peak $\mathrm{f}_{\mathrm{T}}$ for $\mathrm{T} 1$

$\mathrm{T}=150 \mathrm{~K}$. The decrease in the base width with the decrease in temperature decreases the base transient time, resulting in the decrease of the unity gain frequency at around $150 \mathrm{~K}$. For a matter of comparison, we have shown the plot of unity gain frequency versus temperature obtained from the previously reported results using the old BiLow [6] in Fig 30. The unity gain frequency values at $\mathrm{T}=300 \mathrm{~K}$ of Fig 29 is smaller as compared to the corresponding value in Fig 30 . This may be due to the base push out at $\mathrm{T}=300 \mathrm{~K}$. Since the doping in the base is higher in the new transistor $\mathrm{T} 1$ than in the original profile of the Fig 23 , there is more base push out in $\mathrm{T} 1$ and therefore lower $\mathrm{f}_{\mathrm{T}}$ at room temperature. The unity gain frequency as a function of temperature and collector current density is shown in Fig 31 . Fig 32 plots the $\mathrm{f}_{\mathrm{T}}$ as a function of current density as given by the old BiLow [5]. In Fig 32, the unity gain frequency at $\mathrm{T}=300 \mathrm{~K}$ is lower than the frequency at $\mathrm{T}=122 \mathrm{~K}$ whereas in Fig 31 , the $\mathrm{f}_{\mathrm{T}}$ at $\mathrm{T}=300 \mathrm{~K}$ lies between the frequency at $\mathrm{T}=150 \mathrm{k}$ as 
shown in Fig 31 . Fig 32 plots the $\mathrm{f}_{\mathrm{T}}$ as a function of current density as given by the old BiLow [5]. In Fig 32, the unity gain frequency at $\mathrm{T}=300 \mathrm{~K}$ is lower than the frequency at $\mathrm{T}=122 \mathrm{~K}$ whereas in Fig 31 , the $\mathrm{f}_{\mathrm{T}}$ at $\mathrm{T}=300 \mathrm{~K}$ lies between the frequency at $\mathrm{T}=150 \mathrm{k}$ and $\mathrm{T}=122 \mathrm{~K}$. Again we can relate this to the higher doping and smaller size of the transistor T1. In Fig $31, \mathrm{f}_{\mathrm{T}}$ begins to degrade rapidly for temperature below $122 \mathrm{~K}$. The sharp decline of the peak $\mathrm{f}_{\mathrm{T}}$ at low temperature can be explained by the high ratio of the charge trapped on the donor in the base to the charge of the free electrons in the base. The simulated temperature dependence of this ratio, calculated at the peak $f_{\mathrm{T}}$ is shown in Fig 33. It was also observed, from the simulated results, that the total charge at low current densities decreases strongly with an increase in temperature whereas at high current densities it is a weak function of temperature.

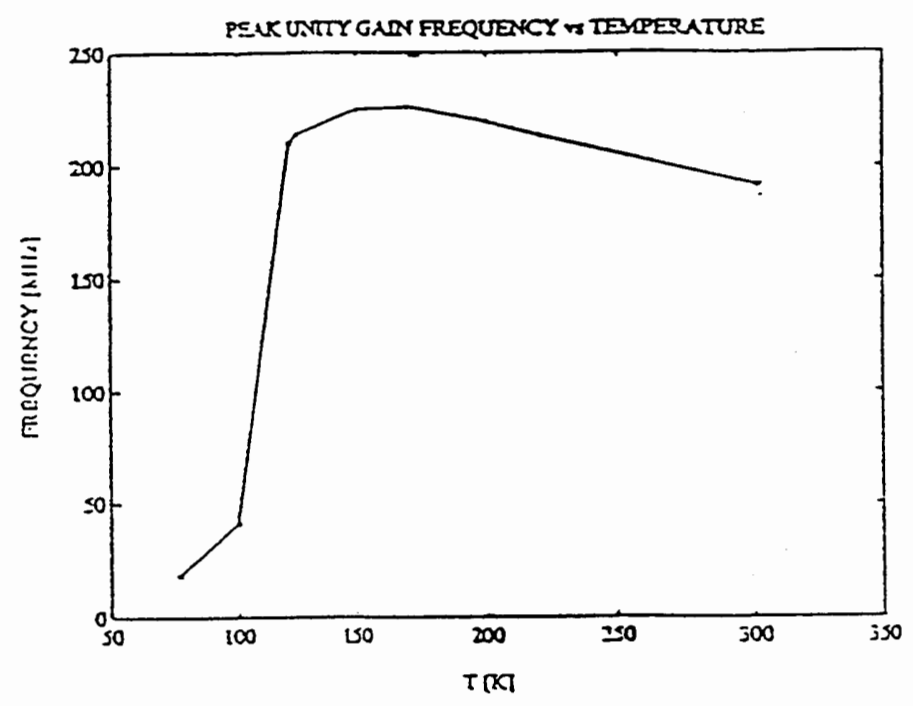

Figure 30. Simulated temperature dependence of peak $f_{T}$ for $n-p-n$ transistor [5] 


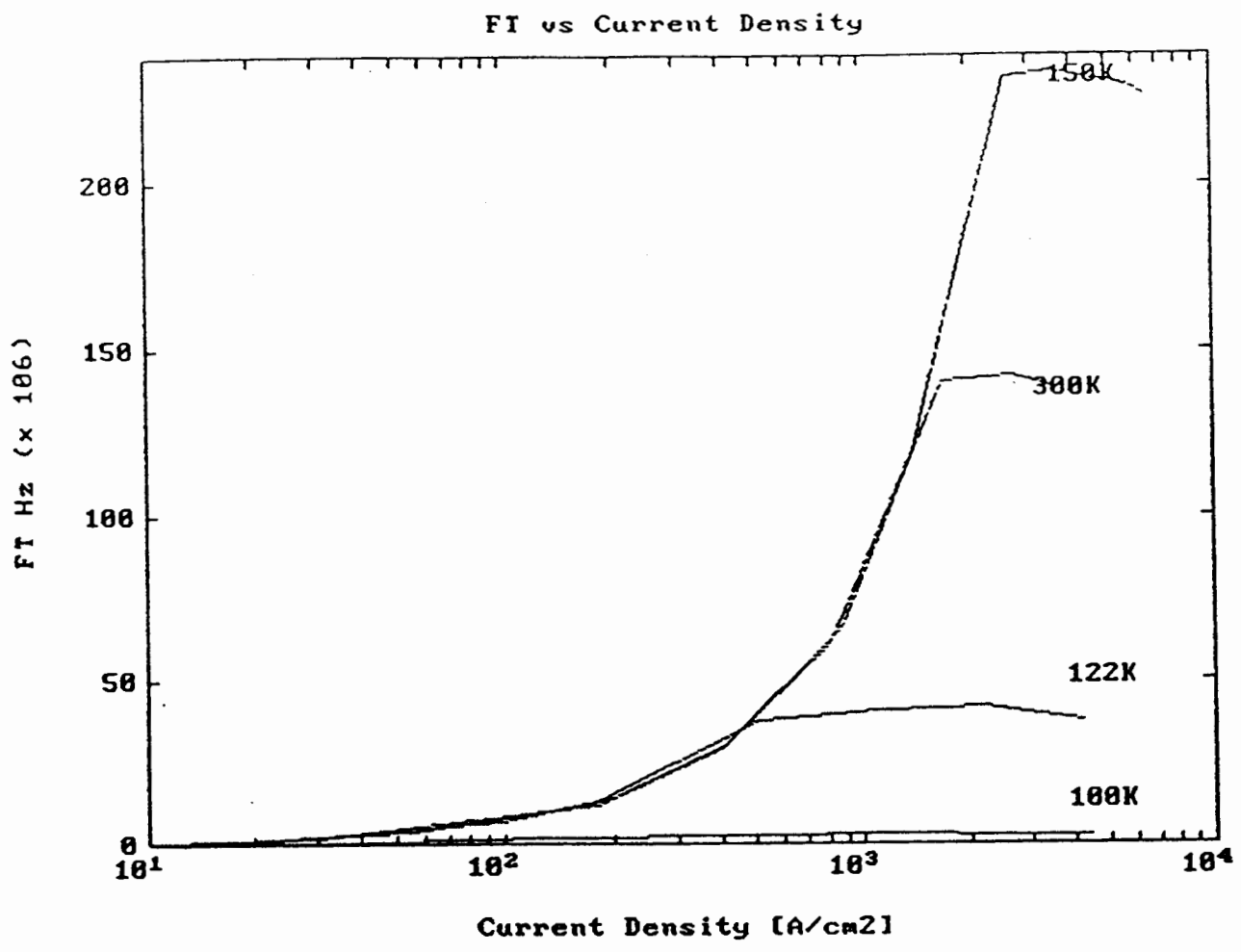

67

Figure 31 Unity gain frequency as a function of collector current at different temperatures for $T 1$.

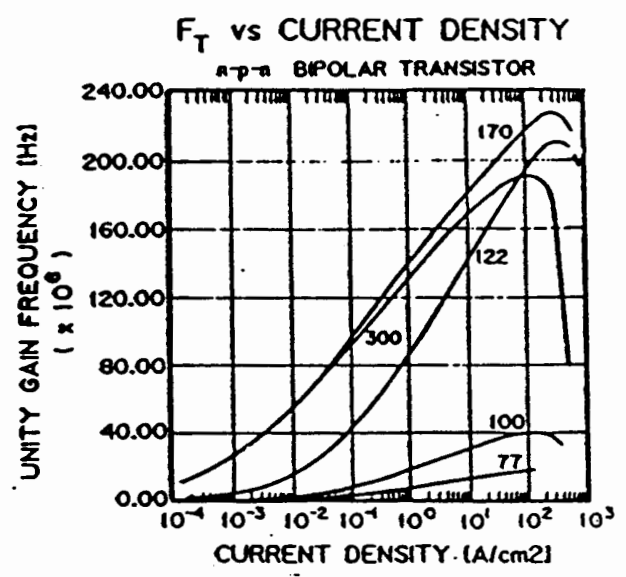

Figure 32 Unity gain frequency as a function of collector current at different temperatures from [5]. 


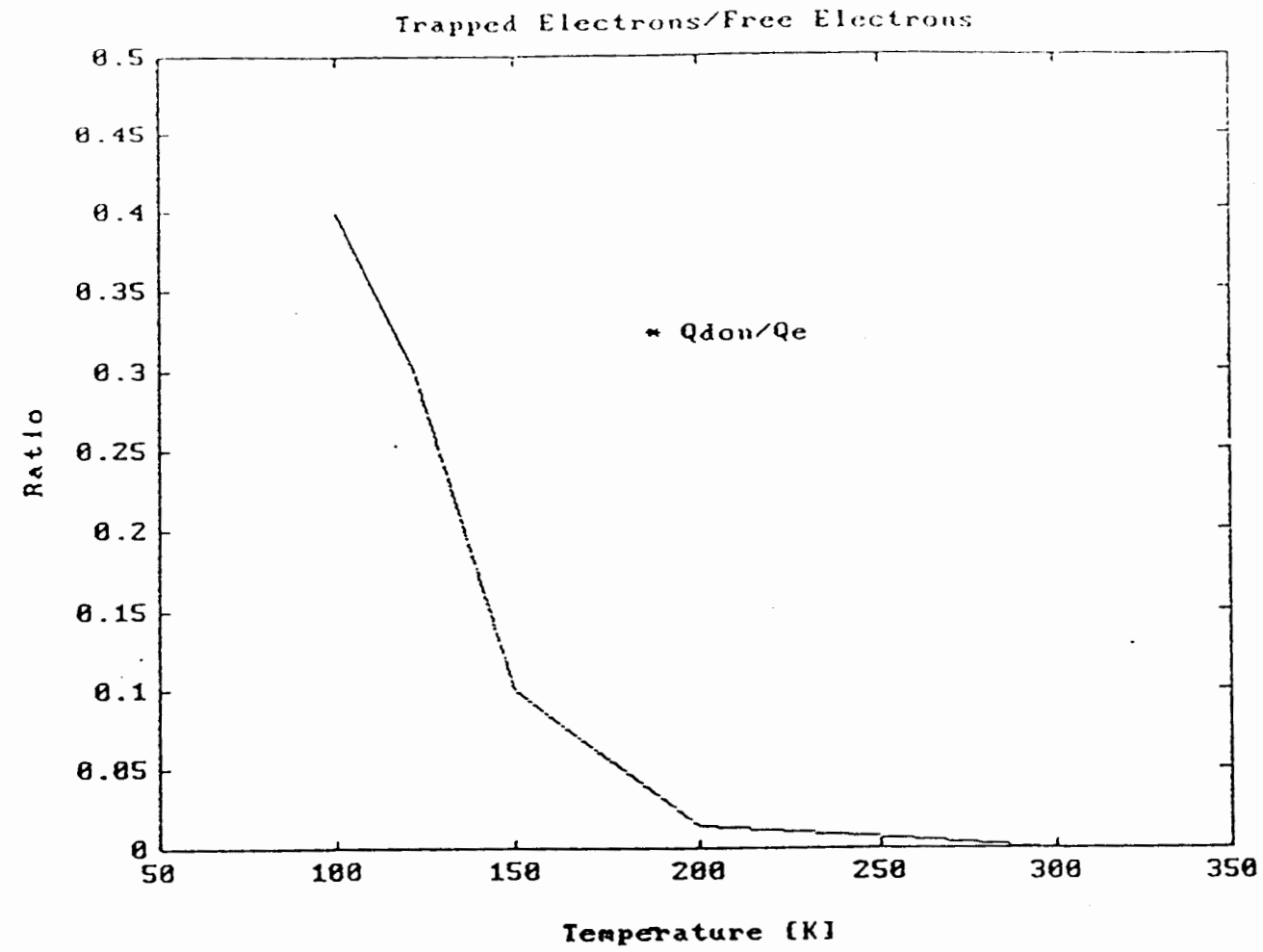

Figure 33 Temperature dependence of the ratio of the charge trapped on the donorrs in the base to the charge of free electrons in the base at peak $\mathrm{f}_{\mathrm{T}}$ for $\mathrm{T} 1$

The degradation of the bipolar transistor performance at low temperature is caused not so much by the difference in the total charge stored in the transistor, as by the temperature dependence between the major components of the charge. This dependence should cause the current gain $\beta$ to increase significantly as a function of collector current density. Unfortunately, the charge of the holes back injected into the emitter becomes dominant charge in the transistor at low temperature for high level injection conditions. This increase nullifies the increase in free electron charge in the base and causes the current gain to decrease. We present in Fig 34 the current gain versus current density and in Fig 35 the current gain versus current density obtained from the old BiLow [5]. Comparing Fig 34 with Fig 35, we see that a there is a large current gain for temperature as low as 


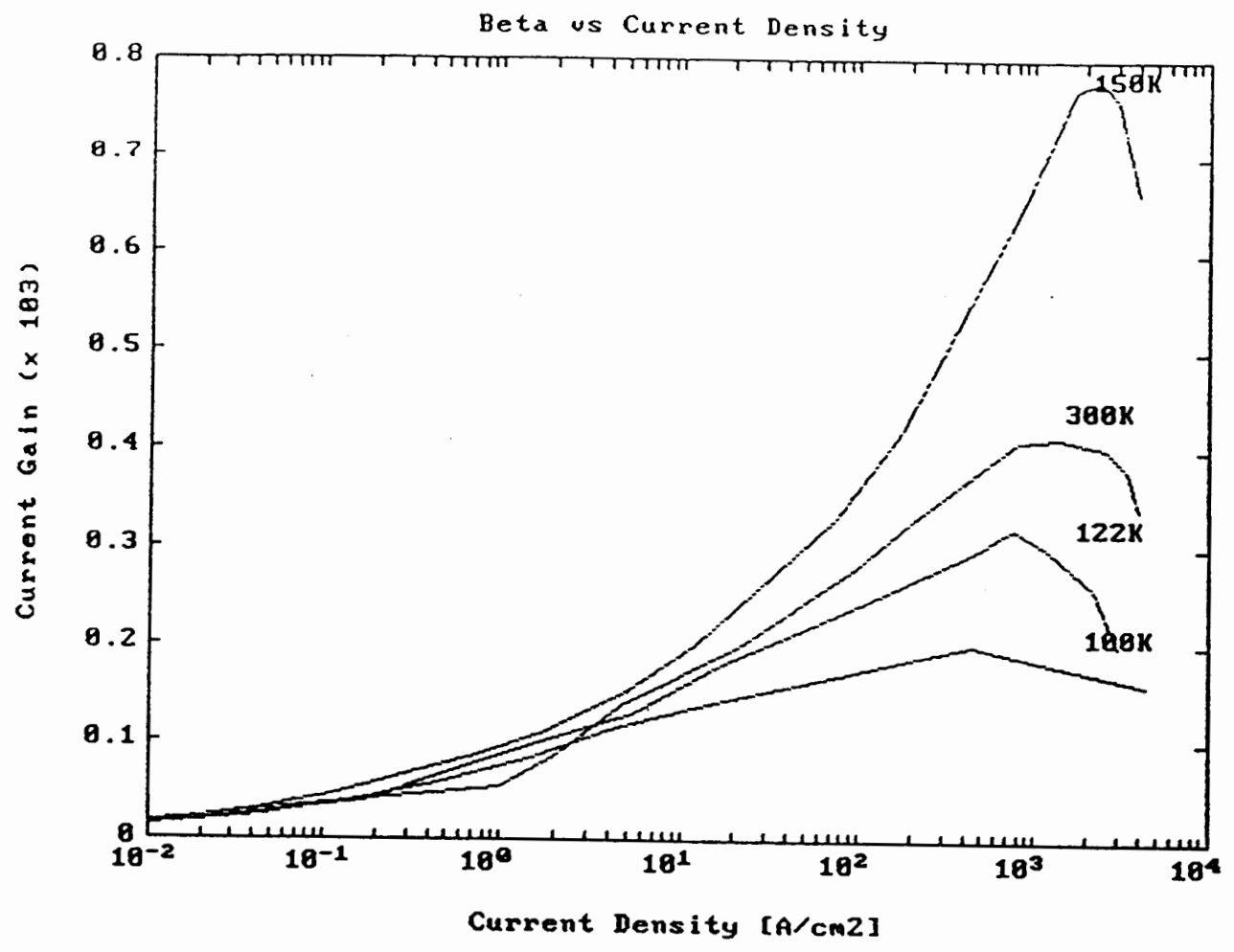

Figure 34 Current-gain as a function of collector current at different temperatures for $\mathrm{T} 1$.

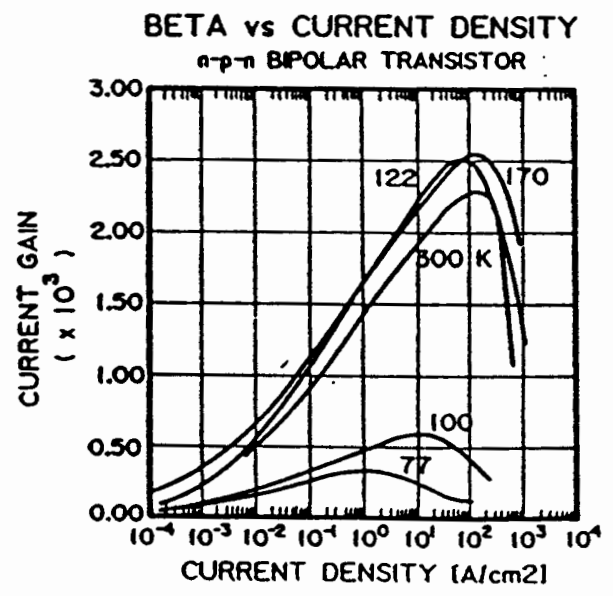

Figure 35 Current-gain as a function of collector current at different temperatures from [5] 

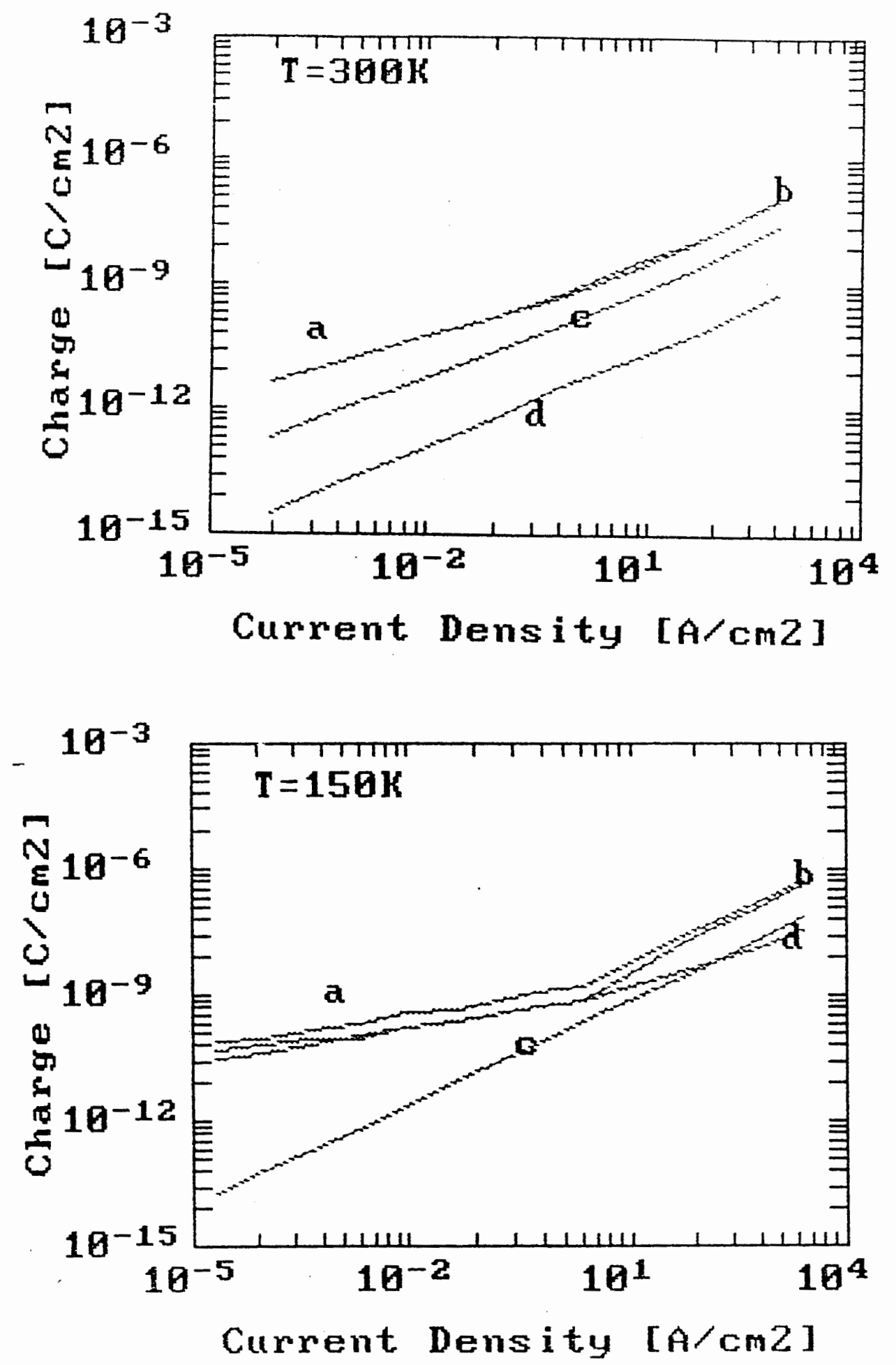

Figure 36. Charge Characteristics at (a) $\mathrm{T}=300 \mathrm{~K}$ and (b) $\mathrm{T}=150 \mathrm{~K}$ a $=$ total charge $b=$ charge of electrons in base, $c=$ charge of holes in emitter, $d=$ donors trapped in base using Method I. 

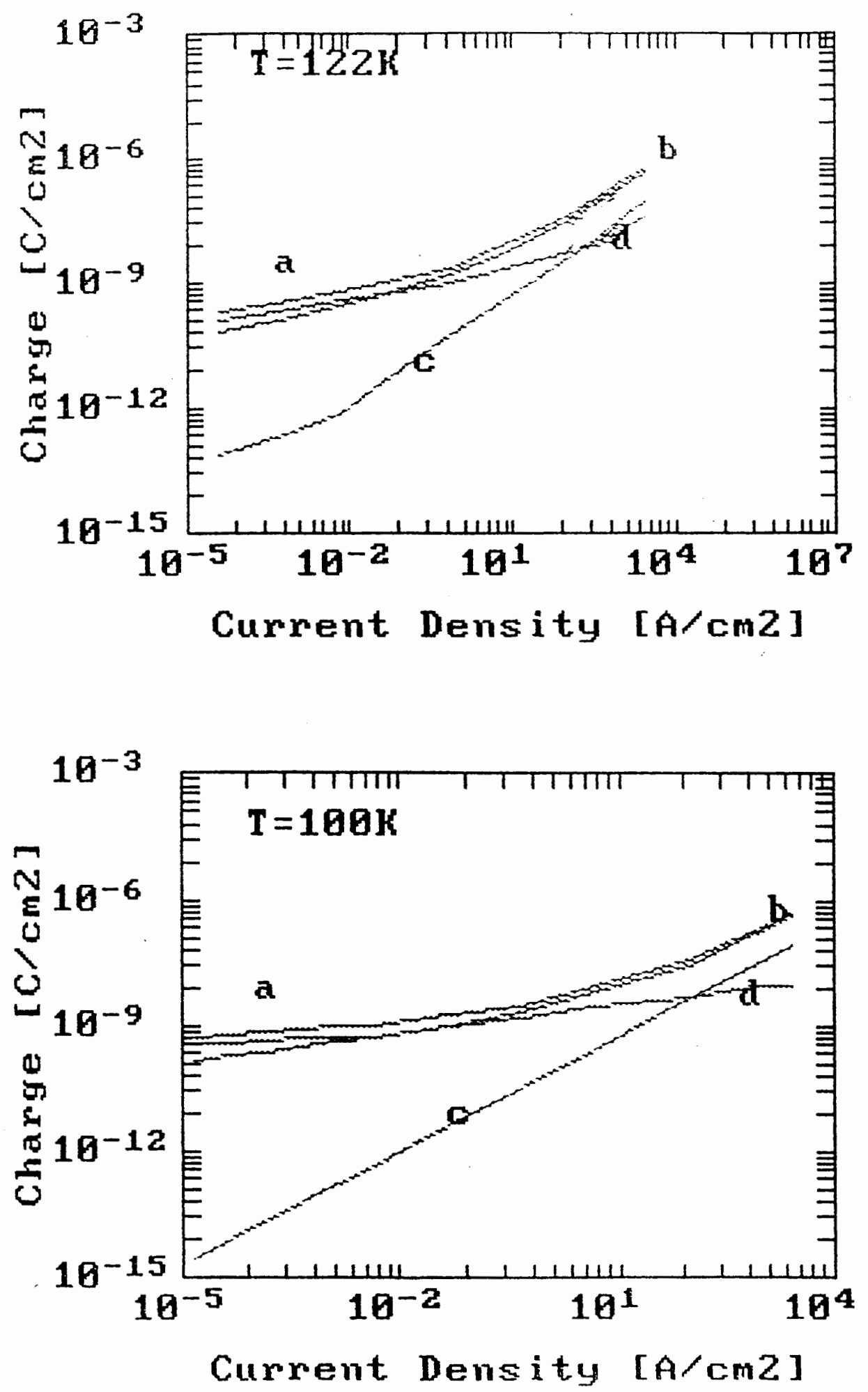

Figure 37. Charge Characteristics at (a) $\mathrm{T}=122 \mathrm{~K}$ and (b) $\mathrm{T}=100 \mathrm{~K}$. $\mathrm{a}=$ total charge $b=$ charge of electrons in base, $c=$ charge of holes in emitter, $d=$ donors trapped in base using Method I. 


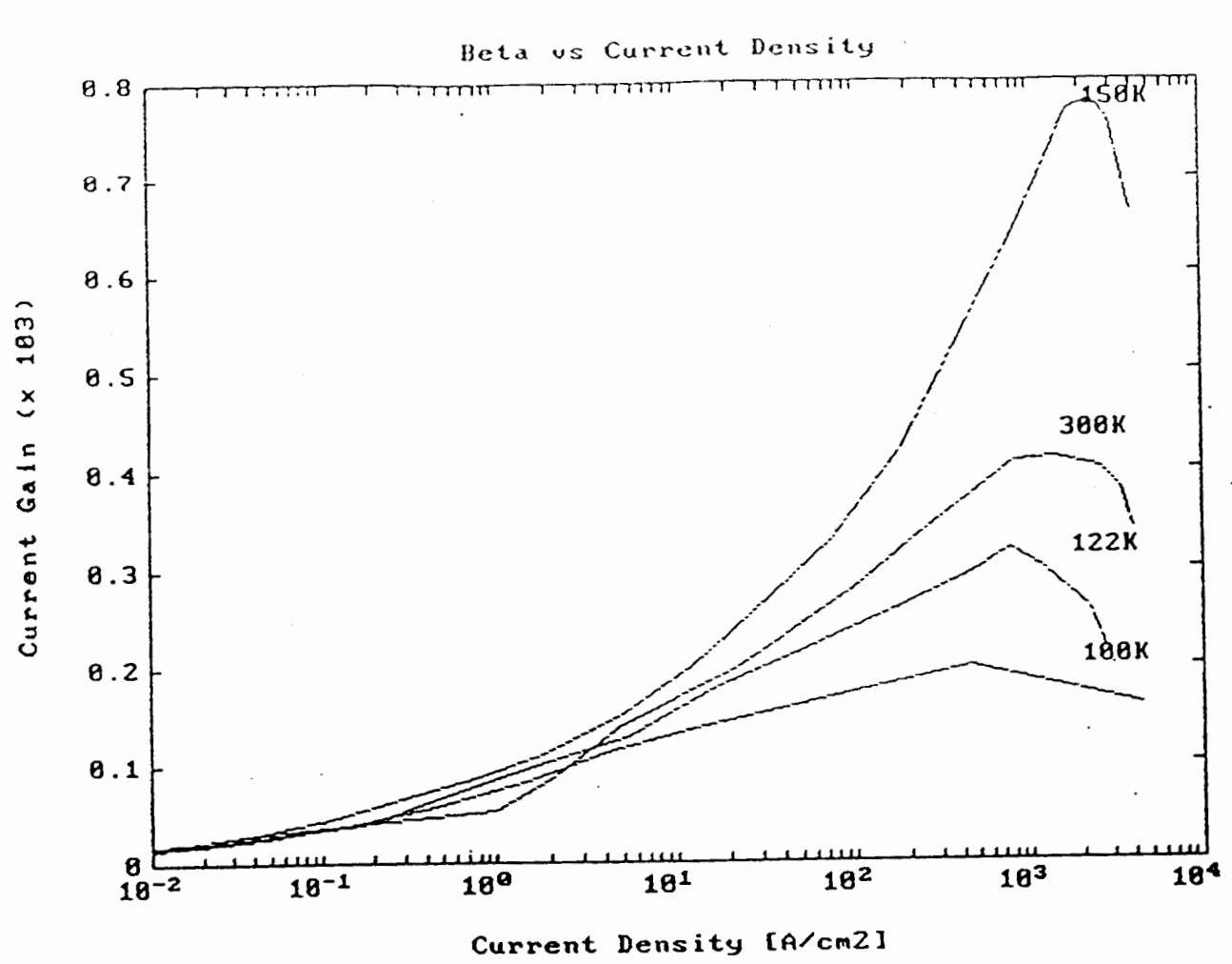

Figure 38. Temperature dependence of $\beta$ using BGN Method I

$122 \mathrm{~K}$ obtained from the simulation results using the old BiLow. Also, the current gain at room temperature is smaller than than the current gain at $\mathrm{T}=122 \mathrm{~K}$ in the old profile of Fig 25 whereas the current gain at room temperature is smaller than the $\beta$ at $\mathrm{T}=150 \mathrm{~K}$ but higher than the $\beta$ at $T=122 \mathrm{~K}$ using $\mathrm{T} 1$. We conclude that this is mainly due to the higher base doping of transistor $\mathrm{T} 1$ which results in a larger base width at room temperature.

Simulations were also performed using the bandgap narrowing models, Method I and Method III, as described in Chapter IV. It was found that for the doping profile shown in Fig 18, the results of the simulations were identical, irrespective of the bandgap model used. The charge versus current density plots using bandgap model I are shown in Fig 36(a)-(b) and Fig 37(a)-(b). The plots of $\beta$ and $f_{T}$ as a function of current density for bandgap Method I are shown in Fig 38 and Fig 39 respectively. The plots of charge, $\beta$ and $f_{\mathrm{T}}$ as a function of current density using bandgap Method III are shown in Fig 40(a)- 
(b), Fig 41(a)-(b), Fig 42 and Fig 43 respectively. One can conclude that the models that were used may have been very close to show any mark difference in the simulation to

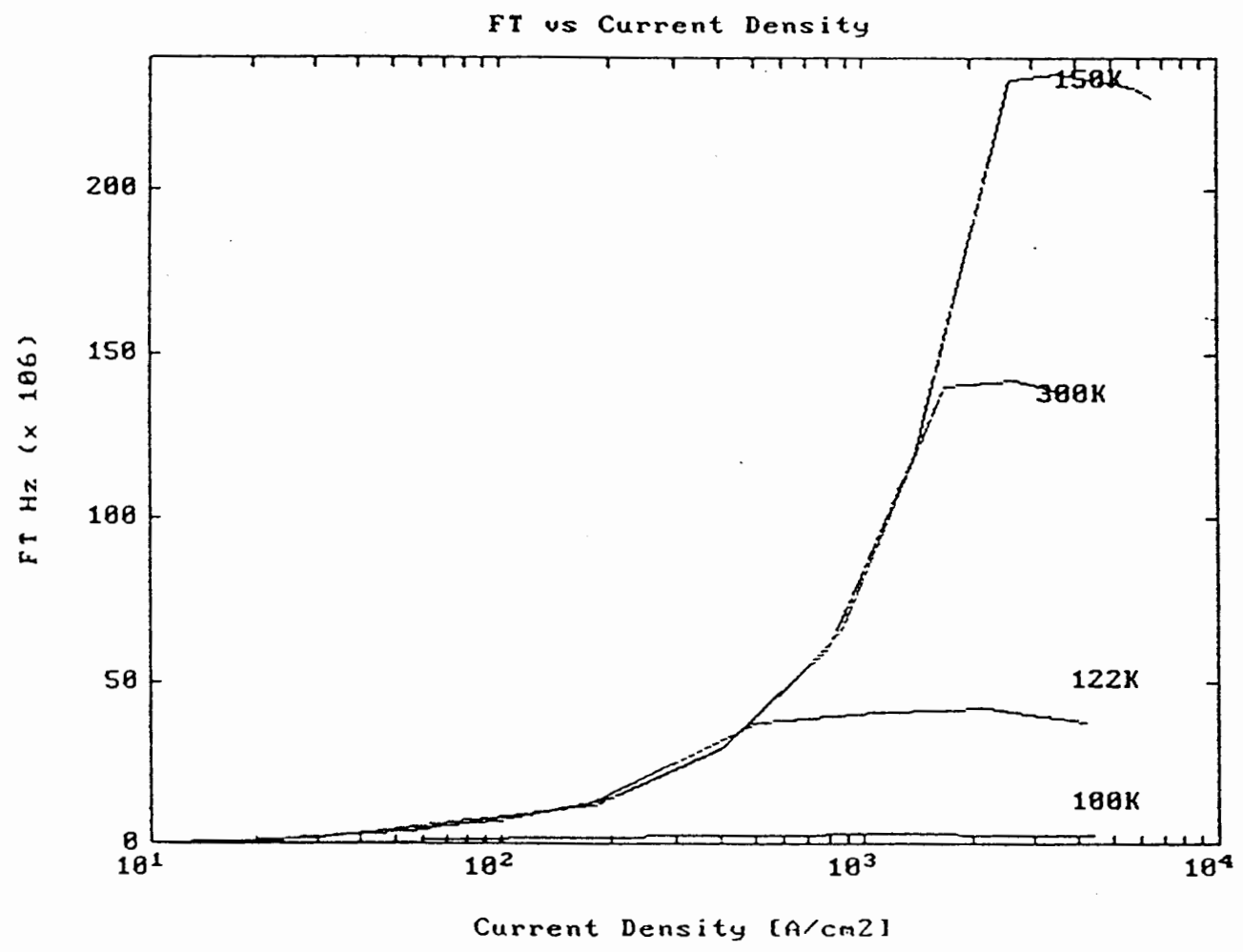

Figure 39. Temperature dependence of $f_{T}$ using BGN Method I

model the BGN effect.

The doping profile of the second transistor is shown in Fig 44. In this profile we have taken into consideration the contribution from the epitaxial layer.

$$
N_{e p i}=N_{b} \exp -\left(\frac{x_{j b c}}{x_{b}}\right)^{2}
$$

The simulations for transistor T2 were performed using bandgap model Method II. The temperature dependence of $\mathrm{f}_{\mathrm{T}}$ is shown in Fig 45. As in Fig 29, the peak $\mathrm{f}_{\mathrm{T}}$ is a function of temperature with a maximum around $\mathrm{T}=150 \mathrm{k}$. However, the corresponding values of 


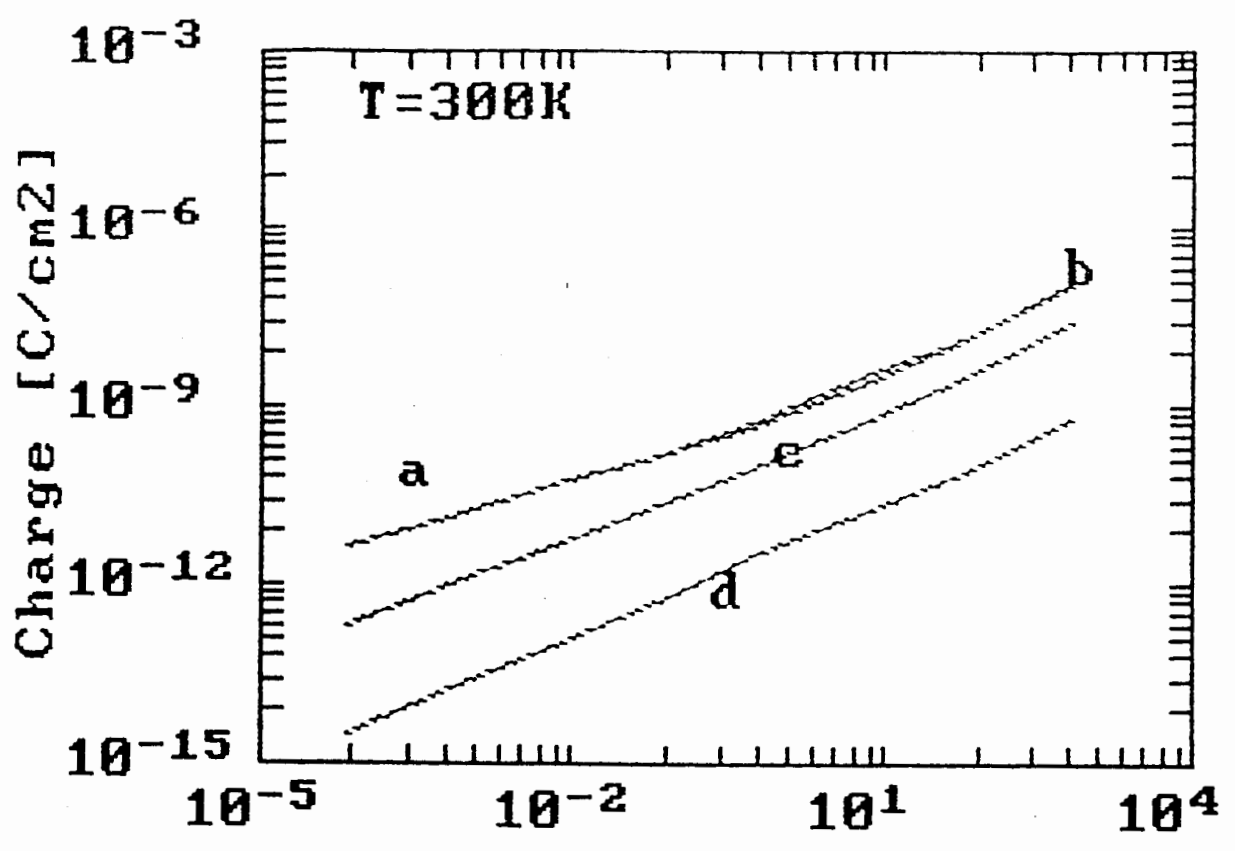

Current Density $[\mathrm{A} / \mathrm{cm} 2]$

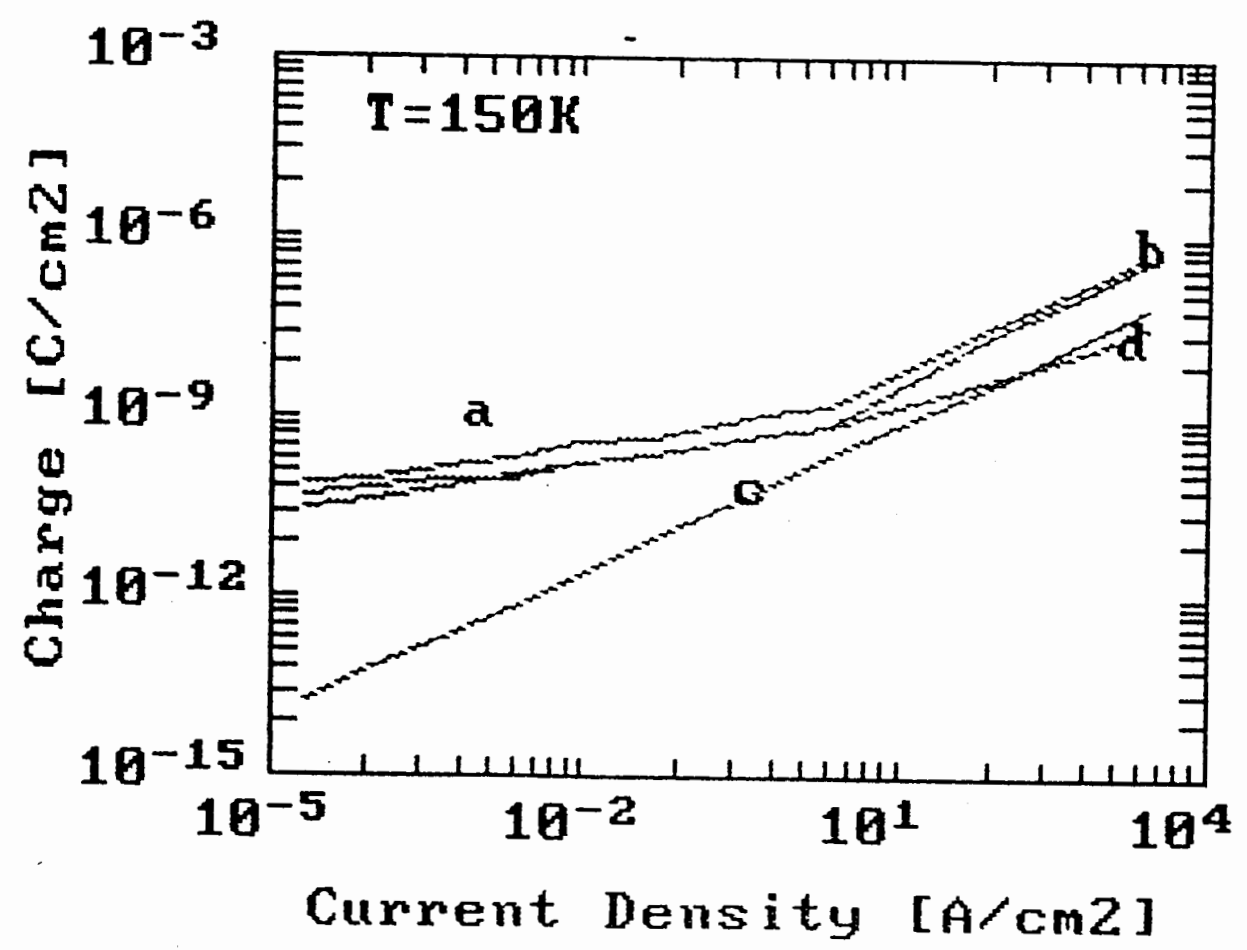

Figure 40. Charge Characteristics at $(a) T=300 \mathrm{~K}$, (b) $\mathrm{T}=150 \mathrm{~K} a=$ total charge, $\mathrm{b}=$ charge of electrons in base, $\mathrm{c}=$ charge of holes in emitter, $\mathrm{d}=$ donors trapped in base, Using Method III. 

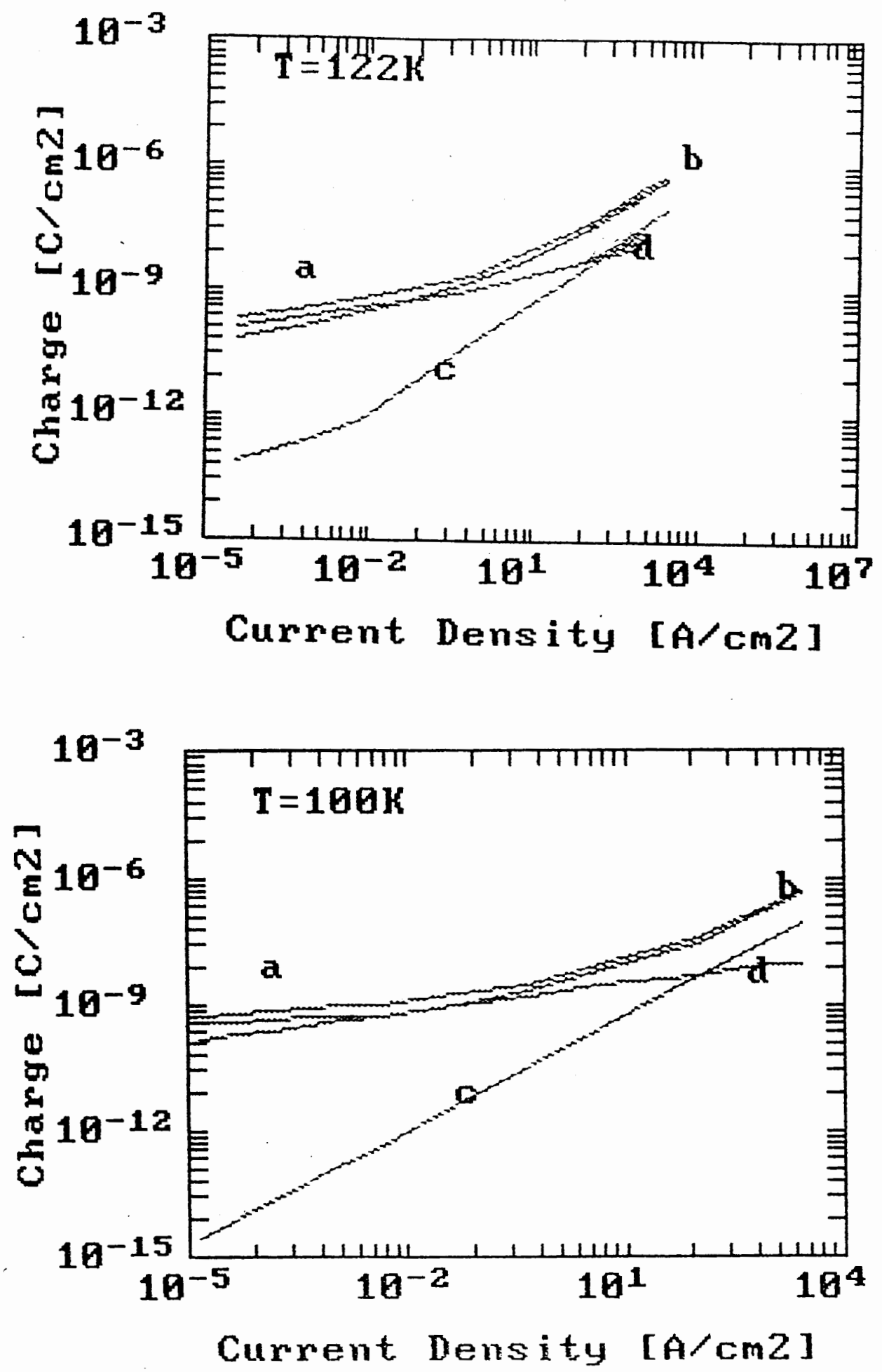

Figure 41.Charge Characteristics at (a) $\mathrm{T}=122 \mathrm{~K}$, (b) $\mathrm{T}=100 \mathrm{~K} \mathrm{a}=$ total charge, $b=$ charge of electrons in base, $c=$ charge of holes in emitter, $d=$ donors trapped in base, Using Method III. 


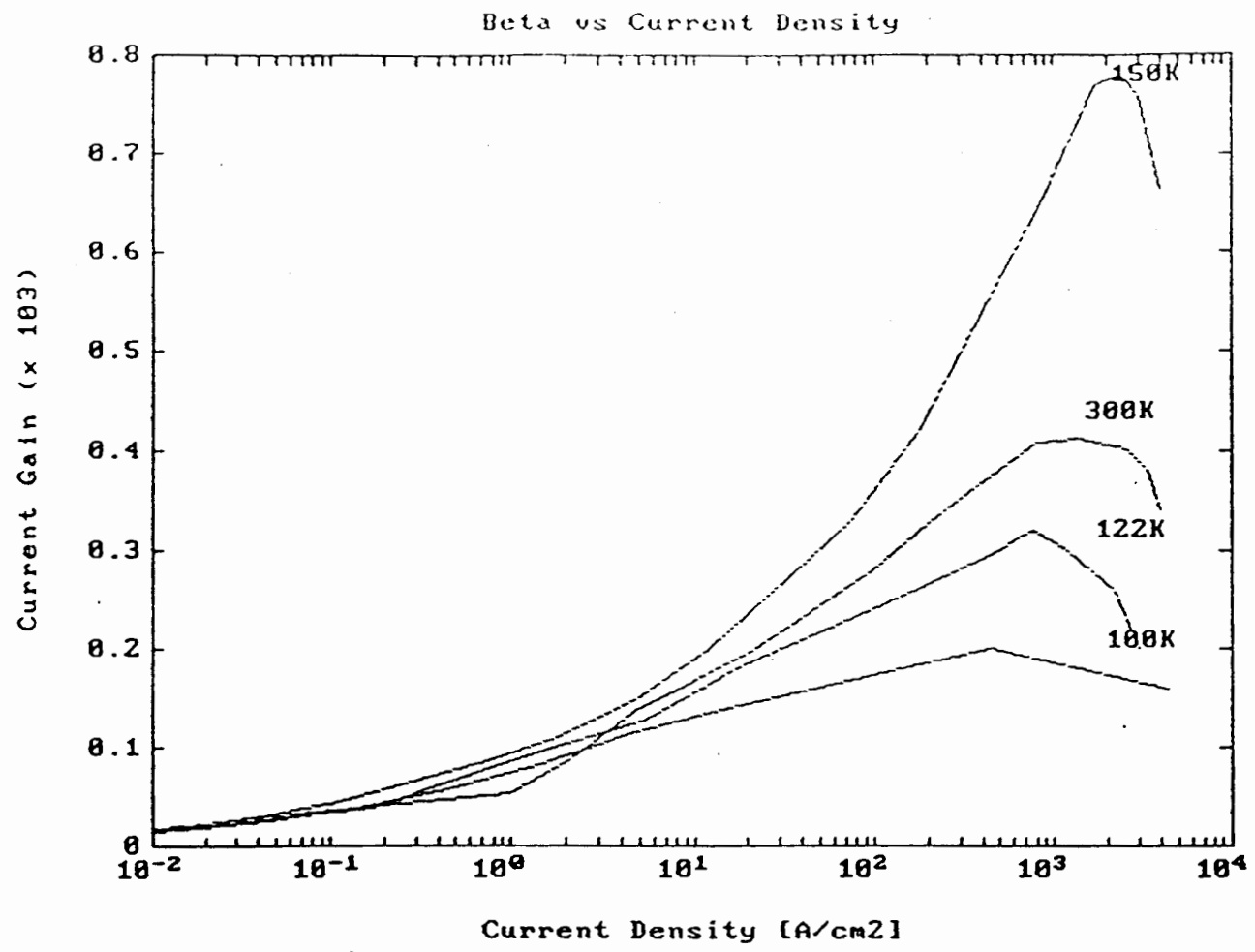

Figure 42. Temperature dependence of $\beta$ using BGN Method III

$\mathrm{f}_{\mathrm{T}}$ are higher than those observed in Fig 29. To reduce the base transient time, we need to minimize the collector resistance $\mathrm{R}_{\mathrm{C}}$. This can be done by using an epitaxial layer doped to withstand breakdown volatage, to be used as a collector. By considering the epitaxial layer in transistor $\mathrm{T} 2$, we have reduced the collector resistance thereby increasing the $\mathrm{f}_{\mathrm{T}}$ and this is evident when we compare the temperature dependence of $f_{\mathrm{T}}$ from the profiles of transistors $\mathrm{T} 1$ and $\mathrm{T} 2$ as shown in Fig 29 and Fig 45. Fig 46(a)-(b) show the simulated charge characteristics as a function of current density at $\mathrm{T}=300 \mathrm{~K}$ and $\mathrm{T}=77 \mathrm{~K}$ for the transistor $\mathrm{T} 2$. For $\mathrm{T}=77 \mathrm{~K}$, the electrons trapped on the donor sites contribute mainly to the total charge for low and medium current densities. However, for high current densities, the charge of the holes back injected into the emitter dominates. Comparing the charge characteristics at $\mathrm{T}=300 \mathrm{~K}$ of Fig 19(a) with the charge of the new transistor $\mathrm{T} 2$ in 
Fig 46(a), we see that the addition of the epilayer has not changed the total charge at room temperature. However, the total charge in the base of transistor T1 and T2 at room temperature, is larger at all values of collector current than that in transistor profile shown in Fig 23 and used in the original BiLow [6]. At $T=77 \mathrm{~K}$, the total charge is mainly due to the charge of the holes back injected into the emitter and there is more charge at $77 \mathrm{~K}$ in $\mathrm{T} 2$ than in the old profile. This can be clearly seen on comparing Fig $46(\mathrm{~b})$ with Fig.26. The main reason for the difference in the amount of charge stored in the transistor is due to higher doping and smaller size of transistor $\mathrm{T} 1$ and $\mathrm{T} 2$ than the transistor profile shown in Fig.23.

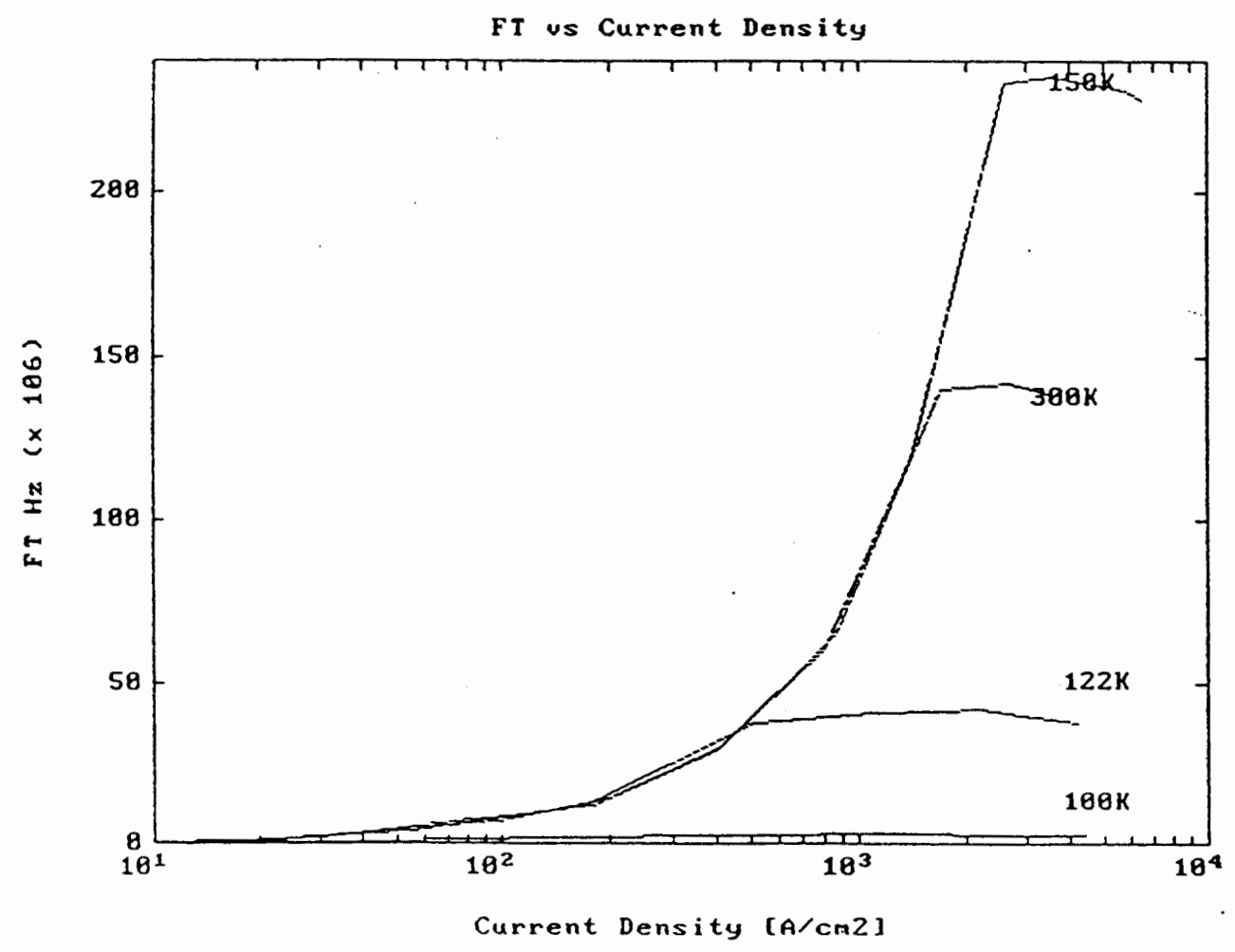

Figure 43. Temperature dependence of $\mathrm{f}_{\mathrm{T}}$ using BGN Method III 


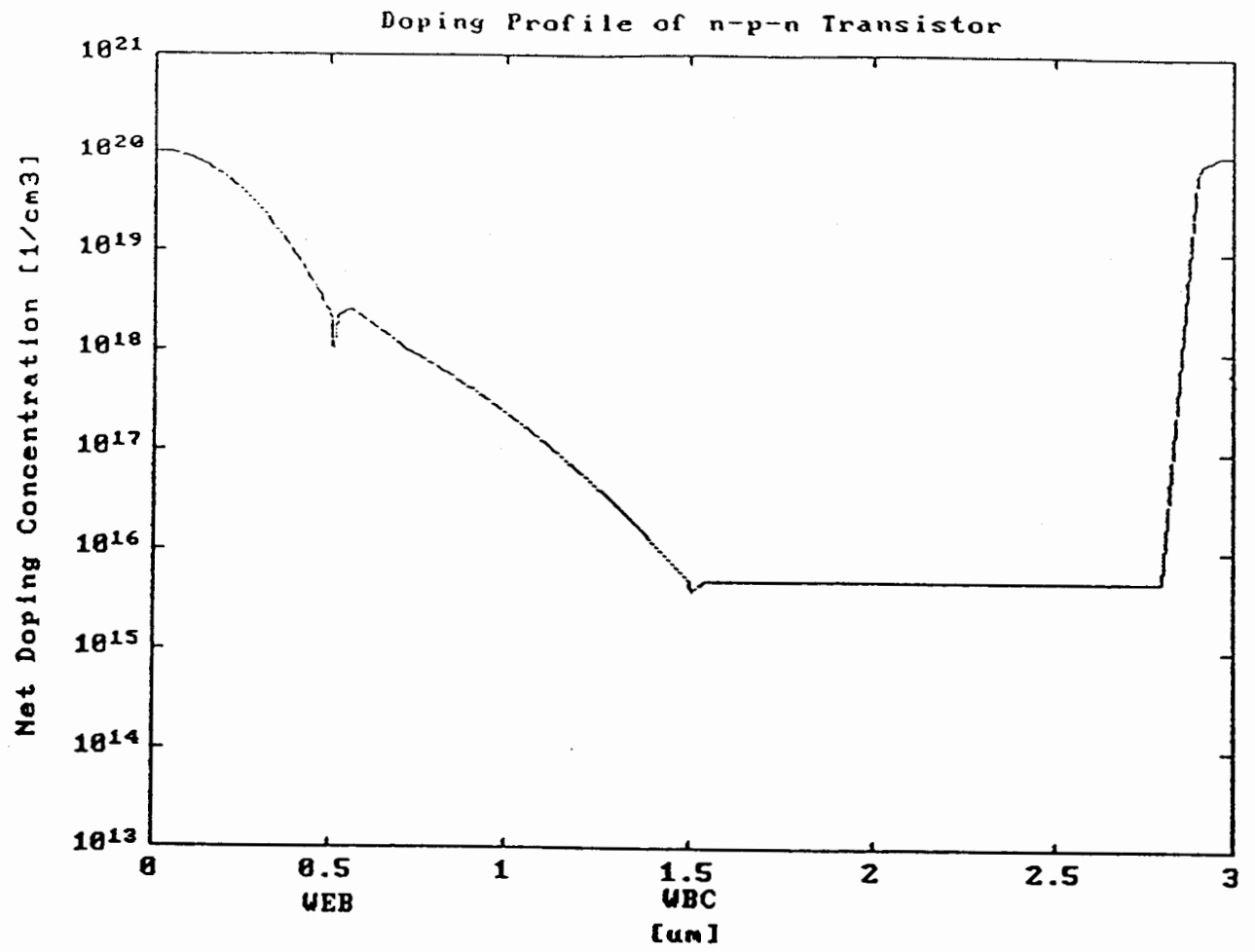

Figure 44. Doping profile of second transistor $T 2$ 


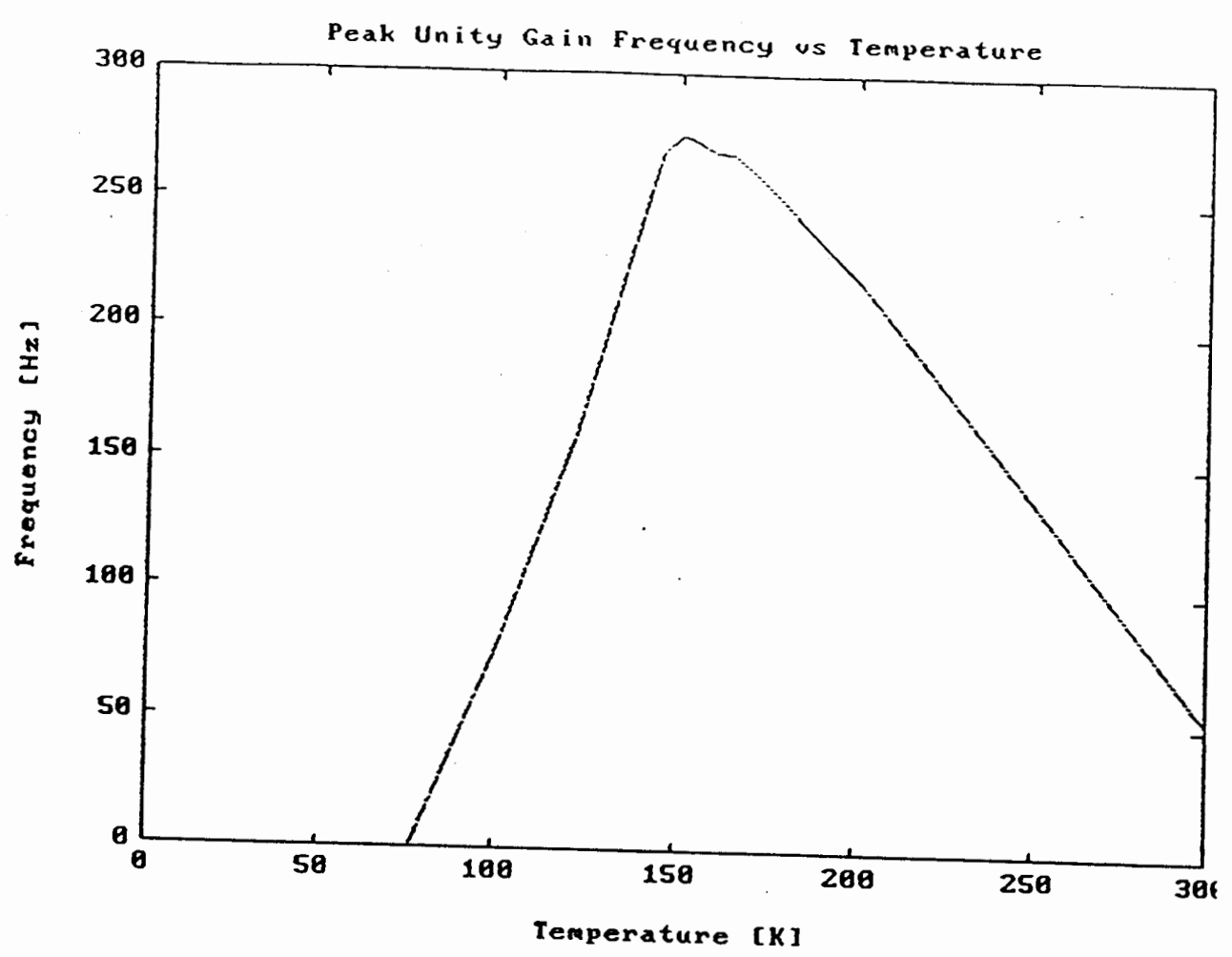

Figure 45. Temperature dependence of $f_{T}$ for $T 2$ 


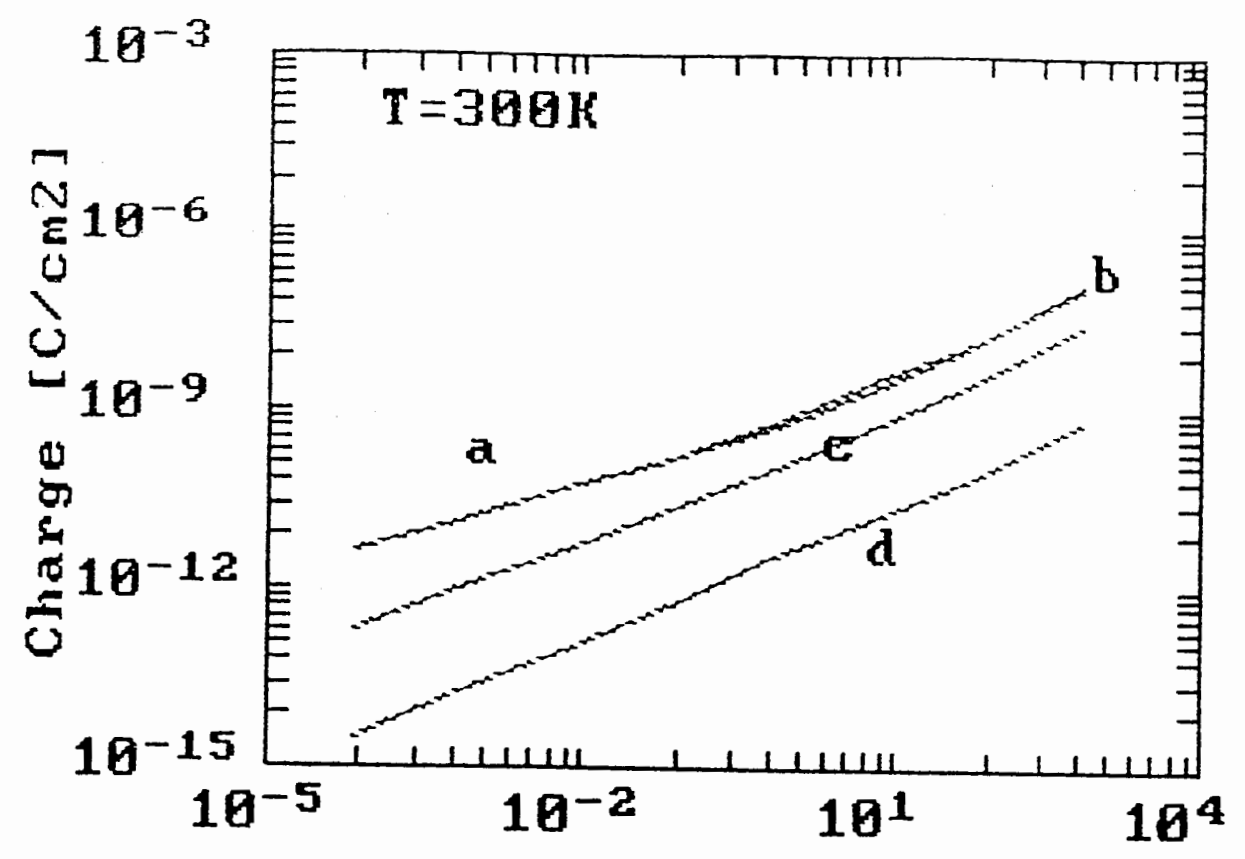

Current Density $[A / \mathrm{cm} Z]$

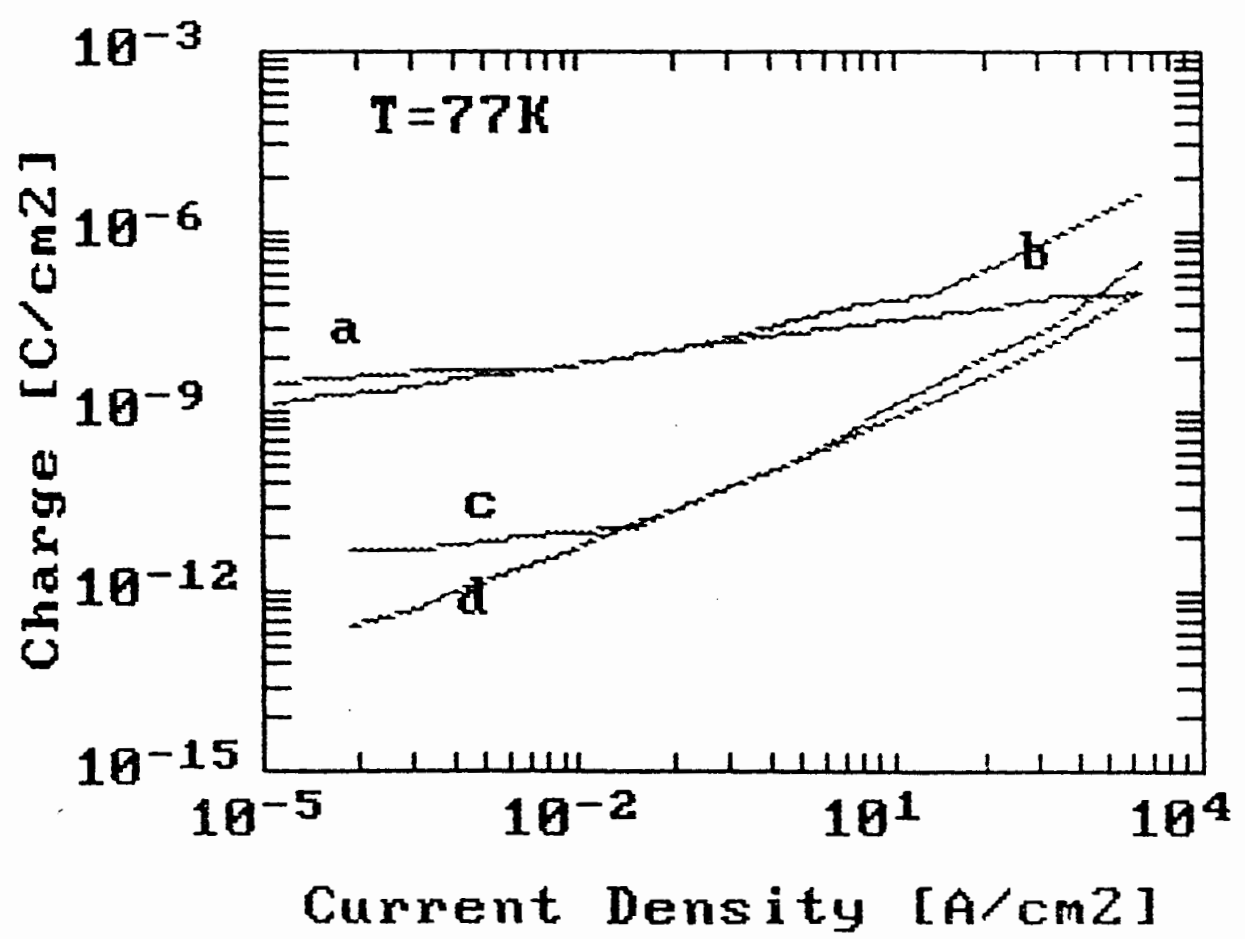

Figure 46. Charge Characteristics at (a) $\mathrm{T}=122 \mathrm{~K}$ and (b) $\mathrm{T}=100 \mathrm{~K}$. $\mathrm{a}=$ total charge, $b=$ charge of electrons in base, $c=$ charge of holes in emitter, $d=$ charge of donors trapped in base for transistor $\mathrm{T} 2$. 


\section{CONCLUSIONS}

A one-dimensional Bipolar transistor simulator has been enhanced for the purpose of proper memory management and easy modification. A model for incomplete ionization and Mott transition originally presented by Kuzmicz [25] has been evaluated at lowtemperature and then included in the simulator. An extensive literature survey on BGN, which is another important parameter for BJT performance at low-temperature, was carried out with the purpose of identifying models with performances in agreement with the experimental data. Two BGN models [27], [10] were selected to be used by the simulator along with the existing model which was modified by adding a Fermi-Dirac correction. The performance of the models was checked by low-temperature simulations of two double diffused n-p-n transistors. Ionization of impurity atoms has also been modeled in the simulator by adding Kuzmicz incomplete ionization model [25].

Simulations have been done using all three of the BGN models. Two $n-p-n$ transistor profiles were simulated with the new BiLow. It was found that the results were identical. This may be due to the fact that the three BGN models used may have been too similar to show any change in simulation results. Also the doping profile in the base could be selected appropriately so as to model the BGN effect. The doping profile that we used had a high concentration in the base. Since the BGN models considered for high doping concentration were almost identical, the simulation results were not affected by these different models After analyzing our BGN results, we prefer to use BGN Method II [27] for our simulations as it is valid for moderate and heavy doping. However, the simulator can select any of the three BGN models at a time. The effect of Mott transition on the abrupt decrease in the electron concentration in emitter has been taken care of by 
smoothing out the concentration profile in the emitter thereby providing a continuity in the region of Mott transition. Both the current gain and the frequency values obtained from simulating the two new profiles were found to be smaller than those from the original BiLow [5] as the doping in the base is higher and the device sizes were smaller. Most of the degradation in $\beta$ and $\mathrm{f}_{\mathrm{T}}$ was found to occur below $150 \mathrm{~K}$. From the charge characteristics plots we find that the total charge which is a strong function of temperature is higher for the profiles studied with the new BiLow than that obtained from the profiles used in the original BiLow simulator[5].

Future application of the BiLow program includes enhancing the current convergence criteria by including the net recombination/generation of carriers at low-temperature. Improving other existing parameters in the simulator, including the heat transfer equations and extending the simulator to two-dimensions. 


\section{REFERENCES}

[1] Barber H.D. " Effective Mass and Intrinsic Concentration in Silicon", Solid State Electron, SC-10 pp.1039-1051 1967.

[2] Bennett H.S., Lowney J.K. "Heavy Doping Effect on Bandgap, Effective Intrinsic Carrier Concentration and Carrier Mobility and Liffetimes", J.Appl.Phys. 527, pp.5533, 1981.

[3] Blaudau W., Onton A., Heinke W. "Temperature Dependence of Bandgap in Silicon", J.Appl .Phys. 45, pp.857-862, 1976.

[4] ChenY.W, Kuo J.B. " Two-Dimensional Analysis of a BiNMOS Transistor Opreating at 77K Using Modified PISCES", IEEE Trans.Electron Devices, ED-36, pp.348-355, 1992.

[5] Chrzanowska-Jeske M., Jaeger R.C. "BiLow-Simulation of Low Temperature Bipolar Transistor Device Behaviour", IEEE Trans.Electron Devices, ED-36, pp.1475-1488, 1989.

[6] Chrzanowska-Jeske M., Jaeger R.C. "Influence of Charge Distribution on Unity Gain Frequency and Current Gain of Silicon Bipolar Transistor For the Temperature Range 77K-300K", Electrochemical Soc.Meeting, Hawai, 1987.

[7] Cressler J.D., Tang D.D., Jenkins K.A., Li G., Yang E.S. "On The Low-Temperature Static And Dynamic Properties Of High-Performance Silicon Bipolar Transistor", Solid State Electron Dvices, ED-36, pp.1489, 1989.

[8] Del Alamo J.A., Swirhun S., Swanson R.M.. "Simultaneous Measurement of Hole Lifetime, Hole Mobility and Bandgap Narrowing in Heavily Doped n-Type Silicon", IEDM, pp.290-293, 1985.

[9] Del Alamo J.A, Swirhun S., Swanson R.M. "Measuring and Modeling MinotityCarrier Transport in Heavily Doped Silicon", Solid-State Electron, 28, pp.47-54, 1985.

[10] Del Alamo J.A, Swanson R.M. "Measurement of Steady-State Minority-Carier 
Transport Parameters in Heavily Doped n-Type Silicon", IEEE Trans. Electron Device", ED-34, pp.1580-1589, July 1987.

[11] De Graff H.C., Klassen F.M. "Compact Transistor Modeling for Circuit Design", Springer, Wein, NewYork, 1990.

[12] DeMari A. "An Accurate Numerical 1-Dimensional Solution of the p-n Junction Under Arbitrary Transient Conditions", Solid-State Electron. SC-11, pp.1021-1053, 1968.

[13] Dumke W.P. "The Effect of Base Doping on the Performance of Si Bipolar Transistor at Low Temperature", IEEE Trans. Electrron Devices, ED-28, pp.494, 1981.

[14] Dumke W.P. "Bandgap Narrowing from Luminescence in p-type Si", Appl.Phys.Lett. 42, pp.196, 1983.

[15] Dziewior J. Silber D. "Minority-Carrier Distribution Coefficient In Heavily Doped Silicon", Appl Phys. Lett. 35, pp.170, 1979.

[16] Fossum J.G, Lee D.S. "Energy Band Distortion in Highly Doped Silicon", Proc.IEDM, pp.316-319, 1979.

[17] Gaensslen F.H., Jaeger R.C.,Walker J.J. " Low Temperature Threshold Behaviour of Depletion Mode Devices", Proc.IEDM, pp.-520-524, 1976.

[18] Gummel H.K. " A Self-Consistant Iterative Scheme For One-Dimensional Steady State Transistor Calculations", IEEE Trans. Electron Devices, ED-11, pp.455465,1964 .

[19] Joyce W.B, Dixon R.W. "Analytical Approximation for the Fermi energy of an Ideal Fermi Gas", Appl Phys. Lett, 31, pp.354-355, 1977.

[20] Jain S.C., Roulston D.J., " A Simple Expression for Bandgap Narrowing (BGN) in Heavily Doped $\mathrm{Si}, \mathrm{Ge}, \mathrm{GaAs}$ and $\mathrm{Ge}_{\mathbf{X}} \mathrm{Si}_{1-\mathrm{x}}$ Strained Layers", Solid-State Electron, SC-34, pp.453-464, 1991.

[21] King R.R., Swanson R.M. "Studies of Diffused Boron Emitters:Saturation Current, Bandgap Narrowing, and Surface Recombination Velocity", IEEE Trans. Electron Devices, ED-38, pp. 1396-1408, 1991.

[22] Kirschman R.K., "Low Temperature Electronics", IEEE Press, Ed. NewYork, 1986. 
[23] Klasssen D.B.M, Slotboom J.W, De Graff H.C. " Unified Apparant Bandgap Narrowing in n- and p-type Silicon", Solid State Electron, SC-35 pp125-129 1992.

[24] Klassen D.B.M. "A Unified Model For Device Simulation-I. Model Equations And Concentration Dependence", Solid-State Electronics, ED-35, pp.953, 1992.

[25] Kuzmicz W. "Ionization of Impurities in Silicon", Solid-state Electronics, SC-29, pp.12223-1227, 1986.

[26] Kuzmicz W. "Modeling of Minority-Carrier Current in Heavily Doped Regions of Bipolar Regions", IEEE Trans.Computer-Aided Design, CAD-5, pp.204-214, 1986.

[27] Kuzmicz W., Zagozdzon-Wosik W. "Heavy Doping Parameters Estimation from Transistor Measurements", Solid-State Electronics, SC-31, pp.91 1-919, 1988.

[28] Lanyon H.P.D., Tuft R.A. "Bandgap Narrowing in Moderately Doped to Heavily Doped Silicon", IEEE Trans. Electron Devices, ED-26, pp. 1014-1018, 979.

[29] Lowney J.R. " Bandgap Narrowing in Space-Charge Region of Heavily Doped Silicon Diodes", Solid-State Electronics SC-28 pp.187, 1985.

[30] Ludwig G.W. Watters R.L. "Drift And Conductivity Mobility In Silicon", Phys.Rev. 101, pp.1699, 1956.

[31] Lu T.C., Kuo J.B. "An Analytical Bandgap Narrowing-Related Current-Gain Model For BJT Devices Operating at 77K", Solid-State Electron, SC-35, pp.785-790, 1992.

[32] Mahan G.D."Energy Gap In Silicon: Impurity Dependence", J.Appl. Phys. 51, pp.2634, 1980.

[33] Mertens R.P, Meerbergen J.L, de Nijs J.F and Overstaeten R.J. " Measurement of the Minority-Carrier Transport Parameter in Heavily Doped Silicon", IEEE Trans.Electron Devices, ED-27, pp.949, 1980.

[34] Mock M.S., "Trransport Equations in Heavily Doped Silicon and the Current Gain of a Bipolar Transistor", Solid-State Electronics, SC-16 pp.1251, 1973.

[35] Morin F.J., Miata J.P. "Electrical Property Of Silicon containing Arsenic And Boron", Phys.Rev. 96, pp.128, 1954.

[36] Neugroschel A., Pao S.C. and Lindholm F.A. "A Method for Determining Energy Gap Narrowing in Highly doped Semiconductors", IEEE Trans Electron Devices, 
ED-29, pp.894, 1982.

[37] Nilsson N.G. "Emperical Approximation for the Fermi Energy for Semiconductors with Parabolic Bands", Appl.Phys.Lett, 33 pp.653 ,1978.

[38] Ning T.H. "Self-Aligned Bipolar Transistor for High Performance and Low-powerDelay VLSI", IEEE Trans.Electron Devices, ED-28, pp.494, 1981.

[39] Park J.S.,Neugroschel A., Lindholm F.A. "A Methodology of Experimentally Based Determination of Gap Shhrinkage and Effective Lifetimes in Emitterand Base of p-n Junction Solar Cells and Other p-n Junction Diodes", IEEE Trans. Electron Devices, ED-29, pp.894, 1986.

[40] Polksy B.S, Rimshans J.S. "Calculation of Effevtive Bandgap Narrowing in Heavily Doped and compensated Silicon", Solid-State Electronics, SC-34, pp.583-586, 1991.

[41] Possin G.E., Adler M.S.,Baliga B.J. " Measurements of the p-n Products in Heavily Doped Epitaxial Emitters", IEEE Trans.Electron Devices, ED-31 pp.3, 1984.

[42] Roulston D.J. "Bipolar Semiconductor devices", McGraw-Hill, NJ 1990.

[43] Satake Hideki, Hamasaki Toshishiko. "Low Temperature (77K) BJT Model with Temperature Dependence on the Injected Condition and Base Resistance", IEEE Trans.Electron Device, ED-17, pp.1688-1697, July 1990.

[44] Schlig E.S. "Low Temperature Operation of Ge Picosecond Logic Cirrcuits", IEEE J.Solid-State Circuits, SC-3, pp.271-276, 1968.

[45] Selberherr S. "Analysis and Simulation of semiconductor Devices", SpringerVerlag, Wien, New York, 1984.

[46] Serneliue B.E. "Bandgap Shift In Heavily Doped n-Type GaAs", Phys Rev. B33, pp.8582, 1986.

[47] Slotboom J.W. "The p-n Product in Silicon", Solid-State Electron, SC-20, pp.279, 1977.

[48] Slotboom J.W, De Graff H.C. "Measurements of Bandgap Narrowing in Si Bipolar Transistors", Solid-State Electronics, SC-19, pp.857-862, 1976.

[49] Swirhun S.E., Kwark Y.H., Swanson R.M. "Bandgap narrowing in heavily doped Si", IEDM Tech.Dig., pp.24 , 1986. 
[50] Sze S.M. "Physics Of Semiconductor Devices", John Wiley \& Sons Inc, 1969.

[51] Tang D.D et al. " High Doping Effects in p-n-p Bipolar Transistors", IEDM Tech. Dig, pp.20,1986

[52] Wagner J. " Bandgap Narrowing in Heavily Doped Silicon at 20K and 300K Studied by Luminescence", Phys Rev, B-299, pp. 2002, 1984.

[53] Wagner J. Solid-State Electron, "Heavily Doped Silicon Studied by Luminescence and Selective Absorption", Solid State Circuits, SC-28, pp.25, 1985.

[54] Wagner J. " Luminescence Studies in Heavily Doped Silicon", Phys Rev. B-32, pp. $1323,1985$.

[55] Wagner J., del Alamo J.A., "Bandgap narrowing in Heavily Doped Silicon: A comparison of Optical and Electrical Data", J.Appl Phys, 63 pp.425-429, 1988.

[56] Wang C.H., Misiakos K., Neugroschel A. " Temperature Dependence Of Minority Carrier Mobility in Heavily Doped Silicon", Appl.Phys. Lett, 57, pp.159, 1990.

[57] Weybright M.E., Plummer J.D. "Bipolar Transistor Modeling Over Temperature", IEEE Trans. Electron Devices, ED-38, 1990.

[58] Wieder A.W. "Emitter Effects in Shallow Bipolar Devices:Measurements and Consequences", IEEE Trans Electron Devices, ED-27, pp.1402, 1980.

[59] Wolf S. "Silicon Processing For The VLSI Era", Vol 2, Lattice Press, CA,1990.

[60] Wolfe C.M. "Physical Properties Of Semiconductor", Prentice-Hall, NJ 1989.

[61] Yano K., Nakazato K., Miyamoto M., Onai T., Aoki M., Shimohigashi K. "A High -Current-Gain Low-Temperature Psuedo Heterojunction Bipolar Transistor Utilizing Sidewall Base-Contact Structure (SICOS)", IEEE Trans. Electron Devices, ED-38, pp.555, 1991. 